Supporting Information for

\title{
5-Exo versus 6-Endo Cyclization of Primary Aminyl Radicals: An Experimental and Theoretical Investigation
}

Feng Liu, Kun Liu, Xinting Yuan and Chaozhong Li*

Joint Laboratory of Green Synthetic Chemistry, Shanghai Institute of Organic Chemistry, Chinese Academy of Sciences, 354 Fenglin Road, Shanghai 200032, P. R. China

\section{clig@mail.sioc.ac.cn}

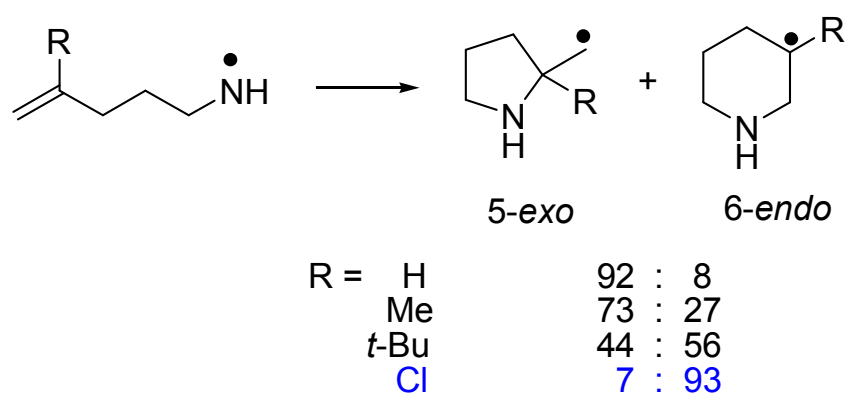

\section{Table of Content}

$\begin{array}{ll}\text { 1. Characterizations of substrates } \mathbf{1}, \mathbf{5} \text { and } \mathbf{9} & \text { S2-S7 }\end{array}$

2. Characterizations of products $\mathbf{2 , 3 , 4 , 6 , 7 , 8 \text { and } \mathbf { 1 0 }} \quad$ S7-S18

$\begin{array}{ll}\text { 3. References for known compounds. } & \text { S18 }\end{array}$

4. Computational results on the cycliczation of aminyl radicals. S19-S45

5. ${ }^{1} \mathrm{H}$ NMR spectra of new compounds. S45-S66 


\section{Characterization of Substrates}

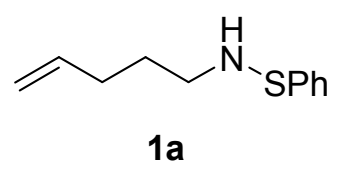

Yield: 55\%. Pale yellow liquid. ${ }^{1} \mathrm{H}$ NMR $\left(300 \mathrm{MHz}, \mathrm{CDCl}_{3}\right) \delta 1.66(2 \mathrm{H}$, quint, $J=7.1 \mathrm{~Hz})$, $2.11(2 \mathrm{H}, \mathrm{q}, J=7.1 \mathrm{~Hz}), 2.86(1 \mathrm{H}, \mathrm{br}), 2.96(2 \mathrm{H}, \mathrm{t}, J=6.4 \mathrm{~Hz}), 4.95-5.06(2 \mathrm{H}, \mathrm{m})$, 5.73-5.85 (1H, m), 7.12-7.35 (5H, m). ${ }^{13} \mathrm{C} \mathrm{NMR}\left(\mathrm{CDCl}_{3}\right) \delta 29.3,30.9,51.4,114.9,123.7$ 125.2, 128.6, 138.0, 141.6. EIMS: $m / z$ (rel intensity) $193\left(\mathrm{M}^{+}, 13\right), 160$ (22), 138 (54), 109 (100), 84 (14), 77 (15), 65 (26), 39 (18). HRMS cacld for $\mathrm{C}_{11} \mathrm{H}_{16} \mathrm{NS}^{+}\left(\mathrm{M}+\mathrm{H}^{+}\right)$: 194.0998 . Found: 194.1000.

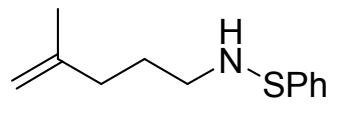

$1 b$

Yield: 76\%. Pale yellow liquid. ${ }^{1} \mathrm{H}$ NMR $\left(300 \mathrm{MHz}, \mathrm{CDCl}_{3}\right) \delta 1.65-1.75(5 \mathrm{H}, \mathrm{m}), 2.06$ $(2 \mathrm{H}, \mathrm{t}, J=7.6 \mathrm{~Hz}), 2.88-2.94(3 \mathrm{H}, \mathrm{m}), 4.68(1 \mathrm{H}, \mathrm{s}), 4.71(1 \mathrm{H}, \mathrm{s}), 7.11-7.34(5 \mathrm{H}, \mathrm{m}) .{ }^{13} \mathrm{C}$ NMR $\left(\mathrm{CDCl}_{3}\right) \delta 22.2,28.1,34.9,51.7,110.2,123.8,125.3,128.6,141.8,145.2$. EIMS: $m / z$ (rel intensity) $208\left(\mathrm{M}^{+}+1,5\right), 192$ (19), 138 (24), 118 (53), 109 (79), 98 (100), 77 (8), 65 (27). HRMS cacld for $\mathrm{C}_{12} \mathrm{H}_{18} \mathrm{NS}^{+}\left(\mathrm{M}^{+}+1\right)$ : 208.1154. Found: 208.1164.

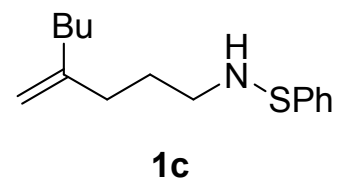

Yield: 54\%. Pale yellow liquid. ${ }^{1} \mathrm{H}$ NMR $\left(300 \mathrm{MHz}, \mathrm{CDCl}_{3}\right) \delta 0.90(3 \mathrm{H}, \mathrm{t}, J=7.0 \mathrm{~Hz})$, 1.26-1.44 (4H, m), 1.69 (2H, quint, $J=7.3 \mathrm{~Hz}), 1.97-2.08(4 \mathrm{H}, \mathrm{m}), 2.87-2.98(3 \mathrm{H}, \mathrm{m})$, $4.71(2 \mathrm{H}, \mathrm{s}), 7.10-7.35(5 \mathrm{H}, \mathrm{m}) .{ }^{13} \mathrm{C}$ NMR $\left(\mathrm{CDCl}_{3}\right) \delta 14.0,22.5,28.4,30.0,33.3,35.7$, 51.9, 109.0, 123.9, 125.4, 128.7, 141.8, 149.4. EIMS: $m / z$ (rel intensity) $249\left(\mathrm{M}^{+}, 3\right), 192$ (43), 140 (100), 138 (40), 109 (66), 94 (20), 55 (19), 41 (24). Anal. calcd for $\mathrm{C}_{15} \mathrm{H}_{23} \mathrm{NS}$ : C, 
72.23; H, 9.29; N, 5.62. Found: C, 72.05; H, 9.19; N, 5.37.

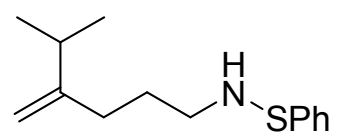

$1 d$

Yield: 52\%. Pale yellow liquid. ${ }^{1} \mathrm{H}$ NMR $\left(300 \mathrm{MHz}, \mathrm{CDCl}_{3}\right) \delta 1.02(6 \mathrm{H}, \mathrm{d}, J=6.9 \mathrm{~Hz})$, 1.70 (2H, quint, $J=7.2 \mathrm{~Hz}), 2.07$ (2H, t $J=7.2 \mathrm{~Hz}), 2.15-2.29(1 \mathrm{H}, \mathrm{m}), 2.87(1 \mathrm{H}, \mathrm{br})$, $2.97(2 \mathrm{H}, \mathrm{q}, J=6.2 \mathrm{~Hz}), 4.68(1 \mathrm{H}, \mathrm{s}), 4.75(1 \mathrm{H}, \mathrm{s}), 7.10-7.34(5 \mathrm{H}, \mathrm{m}) .{ }^{13} \mathrm{C} \mathrm{NMR}\left(\mathrm{CDCl}_{3}\right) \delta$ 21.9, 28.7, 31.6, 33.7, 51.9, 106.7, 123.9, 125.3, 128.7, 141.8, 155.3. EIMS: $m / z$ (rel intensity) $236\left(\mathrm{M}^{+}+1,13\right), 192$ (42), 138 (42), 126 (100), 109 (72), 84 (20), 65 (15), 41 (24). HRMS cacld for $\mathrm{C}_{15} \mathrm{H}_{21} \mathrm{NS}: 235.1395$. Found: 235.1396.

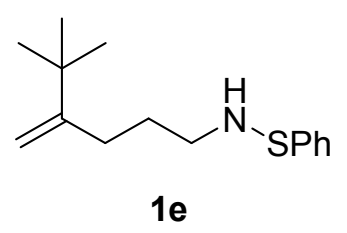

Yield: 51\%. Colorless liquid. ${ }^{1} \mathrm{H}$ NMR $\left(300 \mathrm{MHz}, \mathrm{CDCl}_{3}\right) \delta 1.05(9 \mathrm{H}, \mathrm{s}), 1.73(2 \mathrm{H}$, quint, $J=7.5 \mathrm{~Hz}), 2.08(2 \mathrm{H}, \mathrm{t}, J=7.8 \mathrm{~Hz}), 2.86(1 \mathrm{H}, \mathrm{br}), 2.98(2 \mathrm{H}, \mathrm{t}, J=6.9 \mathrm{~Hz}), 4.68(1 \mathrm{H}, \mathrm{s})$, $4.87(1 \mathrm{H}, \mathrm{s}), 7.11-7.35(5 \mathrm{H}, \mathrm{m}) .{ }^{13} \mathrm{C} \mathrm{NMR}\left(\mathrm{CDCl}_{3}\right) \delta 28.4,29.3,29.9,36.2,52.1,106.1$, 124.0, 125.4, 128.7, 141.8, 157.4. EIMS: $m / z$ (rel intensity) $250\left(\mathrm{M}^{+}+1,19\right), 192$ (33), 140 (100), 138 (41), 109 (68), 84 (81), 65 (14), 41 (24). HRMS cacld for $\mathrm{C}_{15} \mathrm{H}_{23} \mathrm{NS}$ : 249.1551. Found: 249.1555.

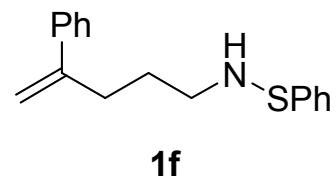

Yield: 59\%. Colorless liquid. ${ }^{1} \mathrm{H}$ NMR (300 MHz, $\left.\mathrm{CDCl}_{3}\right) \delta 1.74(2 \mathrm{H}$, quint, $J=7.2 \mathrm{~Hz}$ ), $2.60(2 \mathrm{H}, \mathrm{t}, J=7.2 \mathrm{~Hz}), 2.87(1 \mathrm{H}, \mathrm{br}), 3.00(2 \mathrm{H}, \mathrm{q}, J=6.2 \mathrm{~Hz}), 5.10(1 \mathrm{H}, \mathrm{s}), 5.32(1 \mathrm{H}, \mathrm{s})$, 7.15-7.44 (10H, m). ${ }^{13} \mathrm{C}$ NMR $\left(\mathrm{CDCl}_{3}\right) \delta 28.8,32.6,51.5,112.7,124.0,125.4,126.2$, 
127.5, 128.4, 128.8, 141.1, 141.8, 147.9. EIMS: $m / z$ (rel intensity) $270\left(\mathrm{M}^{+}+1,1\right), 254$ (4), 160 (100), 138 (14), 109 (38), 91 (17), 77 (11), 65 (12). Anal. calcd for $\mathrm{C}_{17} \mathrm{H}_{19} \mathrm{NS}$ : C, 75.79; H, 7.11; N, 5.20. Found: C, 75.95; H, 7.24; N, 5.05.

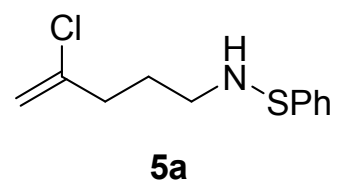

Yield: 46\%. Pale yellow liquid. ${ }^{1} \mathrm{H}$ NMR $\left(300 \mathrm{MHz}, \mathrm{CDCl}_{3}\right) \delta 1.82(2 \mathrm{H}$, quint, $J=7.2 \mathrm{~Hz})$, $2.41(2 \mathrm{H}, \mathrm{t}, J=7.4 \mathrm{~Hz}), 2.89(1 \mathrm{H}, \mathrm{br}), 2.97(2 \mathrm{H}, \mathrm{q}, J=6.1 \mathrm{~Hz}), 5.13-5.15(2 \mathrm{H}, \mathrm{m})$, 7.12-7.35 (5H, m). ${ }^{13} \mathrm{C} \mathrm{NMR}\left(\mathrm{CDCl}_{3}\right) \delta 27.5,36.3,50.5,112.4,123.9,125.4,128.7,141.4$, 142.1. EIMS: $m / z$ (rel intensity) $227\left(\mathrm{M}^{+}, 21\right), 192$ (16), 138 (57), 118 (53), 109 (100), 77 (7), 65 (22), 39 (13). HRMS cacld for $\mathrm{C}_{11} \mathrm{H}_{15} \mathrm{NSCl}(\mathrm{M}+\mathrm{H})$ : 228.0608. Found: 228.0617.

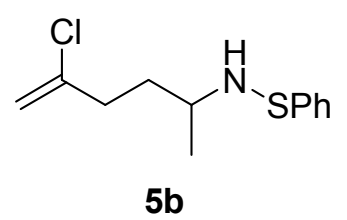

Yield: 90\%. Pale yellow liquid. ${ }^{1} \mathrm{H}$ NMR $\left(300 \mathrm{MHz}, \mathrm{CDCl}_{3}\right) \delta 1.16(3 \mathrm{H}, \mathrm{d}, J=6.6 \mathrm{~Hz})$, 1.61-1.73 (1H, m), 1.78-1.90 (1H, m), $2.40(2 \mathrm{H}, \mathrm{t}, J=7.5 \mathrm{~Hz}), 2.81$ (1H, br), 2.93-3.01 $(1 \mathrm{H}, \mathrm{m}), 5.09-5.12(2 \mathrm{H}, \mathrm{m}), 7.09-7.14(1 \mathrm{H}, \mathrm{m}), 7.27-7.31(4 \mathrm{H}, \mathrm{m}) .{ }^{13} \mathrm{C} \mathrm{NMR}\left(\mathrm{CDCl}_{3}\right) \delta$ 20.7, 34.7, 35.6, 55.0, 112.1, 123.6, 125.23, 128.6, 142.4, 142.5. EIMS: $\mathrm{m} / \mathrm{z}$ (rel intensity) $\left(\mathrm{M}^{+}, 6\right), 206$ (5), 152 (81), 132 (42), 109 (100), 93 (30), 77 (17), 65 (36). Anal. calcd for $\mathrm{C}_{12} \mathrm{H}_{16} \mathrm{ClNS}: \mathrm{C}, 59.61 ; \mathrm{H}, 6.67 ; \mathrm{N}, 5.79$. Found: C, 59.74; H, 6.54; N, 6.10.

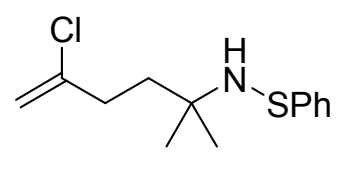

$5 c$

Yield: 97\%. Pale yellow liquid. ${ }^{1} \mathrm{H}$ NMR $\left(300 \mathrm{MHz}, \mathrm{CDCl}_{3}\right) \delta 1.16(6 \mathrm{H}, \mathrm{s}), 1.73-1.78(2 \mathrm{H}$, m), 2.33-2.39 $(2 \mathrm{H}, \mathrm{m}), 2.83(1 \mathrm{H}, \mathrm{s}), 5.04(1 \mathrm{H}, \mathrm{s}), 5.10(1 \mathrm{H}, \mathrm{s}), 7.08(1 \mathrm{H}, \mathrm{t}, J=7.0 \mathrm{~Hz})$, 
7.28-7.37 (4H, m). ${ }^{13} \mathrm{C} \mathrm{NMR}\left(\mathrm{CDCl}_{3}\right) \delta 26.9,34.5,39.3,56.7,111.7,122.9,124.7,128.4$, 143.1, 143.9. EIMS: $m / z$ (rel intensity) $255\left(\mathrm{M}^{+}, 5\right), 166$ (38), 125 (100), 109 (35), 93 (38), 77 (11), 65 (17), 42 (16). HRMS cacld for $\mathrm{C}_{12} \mathrm{H}_{18}$ CINS: 255.0848. Found: 255.0854.

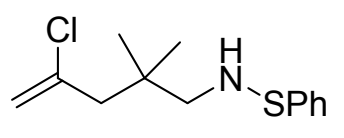

5d

Yield: 66\%. Pale yellow liquid. ${ }^{1} \mathrm{H}$ NMR $\left(300 \mathrm{MHz}, \mathrm{CDCl}_{3}\right) \delta 0.99(6 \mathrm{H}, \mathrm{s}), 2.36(2 \mathrm{H}, \mathrm{s})$, $2.88(3 \mathrm{H}, \mathrm{s}), 5.10(1 \mathrm{H}, \mathrm{s}), 5.28(1 \mathrm{H}, \mathrm{s}), 7.10-7.34(5 \mathrm{H}, \mathrm{m}) .{ }^{13} \mathrm{C} \mathrm{NMR}\left(\mathrm{CDCl}_{3}\right) \delta 25.4,36.0$, 48.1, 63.3, 115.9, 123.7, 125.4, 128.8, 139.5, 142.4. EIMS: $m / z$ (rel intensity) $256\left(\mathrm{M}^{+}+1\right.$, 17), 220 (8), 146 (73), 138 (100), 109 (94), 94 (29), 77 (11), 65 (23). HRMS cacld for $\mathrm{C}_{12} \mathrm{H}_{18} \mathrm{ClNS}: 255.0848$. Found: 255.0847.

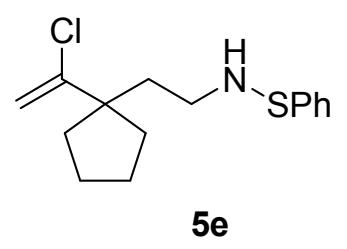

Yield: $72 \%$. Pale yellow liquid. ${ }^{1} \mathrm{H}$ NMR $\left(300 \mathrm{MHz}, \mathrm{CDCl}_{3}\right) \delta 1.50-1.56(2 \mathrm{H}, \mathrm{m})$, 1.61-1.68 (4H, m), 1.82-1.92 (4H, m), 2.80-1.91 (3H, m), 5.10-5.21 (2H, m), 7.10-7.34 $(5 \mathrm{H}, \mathrm{m}) .{ }^{13} \mathrm{C} \mathrm{NMR}\left(\mathrm{CDCl}_{3}\right) \delta 23.2,36.8,38.1,48.9,53.4,111.7,123.8,125.3,128.7$, 141.7, 148.6. EIMS: $m / z$ (rel intensity) $282\left(\mathrm{M}^{+}+1,2\right), 246(9), 172$ (41), 138 (47), 109 (100), 77 (28), 65 (47), 41 (40). HRMS cacld for $\mathrm{C}_{15} \mathrm{H}_{20} \mathrm{ClNS}$ : 281.1005. Found: 281.1012 .

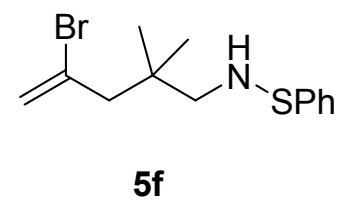

Yield: 77\%. Pale yellow liquid. ${ }^{1} \mathrm{H}$ NMR $\left(300 \mathrm{MHz}, \mathrm{CDCl}_{3}\right) \delta 1.01(6 \mathrm{H}, \mathrm{s}), 2.51(2 \mathrm{H}, \mathrm{s})$, 
$2.89(3 \mathrm{H}, \mathrm{s}), 5.55(2 \mathrm{H}, \mathrm{s}), 7.14-7.35(5 \mathrm{H}, \mathrm{m}) .{ }^{13} \mathrm{C} \mathrm{NMR}\left(\mathrm{CDCl}_{3}\right) \delta 25.4,36.3,50.0,63.2$, 120.7, 123.8, 125.4, 128.8, 129.7, 142.3. EIMS: $m / z$ (rel intensity) $302\left(\mathrm{M}^{+}\left({ }^{81} \mathrm{Br}\right)+1\right.$, 5)/300 (M+ $\left.\left({ }^{79} \mathrm{Br}\right)+1,4\right), 220$ (32), 190 (9), 138 (92), 109 (100), 94 (30), 65 (25), 41 (29). HRMS cacld for $\mathrm{C}_{13} \mathrm{H}_{18} \mathrm{BrNS}: 299.0343$. Found: 299.0358.

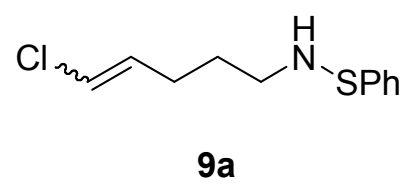

Yield: 79\%. Pale yellow liquid. $Z: E=63: 37 .{ }^{1} \mathrm{H}$ NMR $\left(300 \mathrm{MHz}, \mathrm{CDCl}_{3}\right) \delta 1.62-1.72(2 \mathrm{H}$, m), 2.11/2.29 (2H, q, $J=7.2), 2.91-3.00(3 \mathrm{H}, \mathrm{m}), 5.73-6.05(2 \mathrm{H}, \mathrm{m}), 7.14-7.35(5 \mathrm{H}, \mathrm{m})$. ${ }^{13} \mathrm{C} \quad \mathrm{NMR}\left(\mathrm{CDCl}_{3}\right) \quad \delta \quad 24.3 / 28.1,28.8 / 29.2, \quad 51.0 / 51.3, \quad 117.4 / 118.6, \quad 123.9 / 124.1$, 125.4/125.5, 128.75/128.78, 130.9, 133.1/141.7. EIMS: $m / z$ (rel intensity) $227\left(\mathrm{M}^{+}, 8\right)$, 192 (10), 138 (31), 109 (100), 82 (18), 70 (57), 65 (27), 53 (17). Anal. calcd for $\mathrm{C}_{11} \mathrm{H}_{14} \mathrm{ClNS}: \mathrm{C}, 58.01 ; \mathrm{H}, 6.20$; N, 6.15. Found: C, 58.46; H, 6.36; N, 6.19.

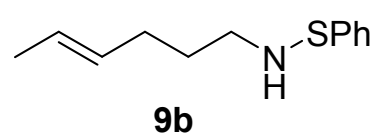

Yield: 57\%. Pale yellow liquid. ${ }^{1} \mathrm{H}$ NMR $\left(300 \mathrm{MHz}, \mathrm{CDCl}_{3}\right) \delta 1.56-1.65(5 \mathrm{H}, \mathrm{m}), 2.03$ (2H, q, $J=6.5), 2.84(1 \mathrm{H}, \mathrm{br}), 2.93(2 \mathrm{H}, \mathrm{q}, J=6.4), 5.33-5.48(2 \mathrm{H}, \mathrm{m}), 7.10-7.34(5 \mathrm{H}, \mathrm{m})$. ${ }^{13} \mathrm{C}$ NMR $\left(\mathrm{CDCl}_{3}\right) \delta 17.9,29.9,30.1,51.7,123.9,125.3,125.5,128.8,130.6,141.9$. EIMS: m/z (rel intensity) $207\left(\mathrm{M}^{+}, 4\right), 138$ (33), 125 (8), 109 (98), 94 (22), 70 (100), 65 (27), 41 (26). HRMS cacld for $\mathrm{C}_{12} \mathrm{H}_{17} \mathrm{NS}: 207.1082$. Found: 207.1090. Anal. calcd for $\mathrm{C}_{12} \mathrm{H}_{17} \mathrm{NS}$ : C, 69.51; H, 8.26; N,6.76. Found: C, 69.63; H, 7.85; N, 6.45.

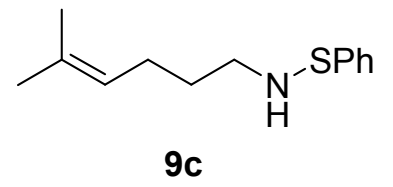

Yield: 68\%. Pale yellow liquid. ${ }^{1} \mathrm{H}$ NMR $\left(300 \mathrm{MHz}, \mathrm{CDCl}_{3}\right) \delta 1.55-1.65(5 \mathrm{H}, \mathrm{m}), 1.69$ 
$(3 \mathrm{H}, \mathrm{s}), 2.03(2 \mathrm{H}, \mathrm{q}, J=7.3), 2.85(1 \mathrm{H}, \mathrm{br}), 2.94(2 \mathrm{H}, \mathrm{q}, J=6.2), 5.08-5.13(1 \mathrm{H}, \mathrm{m})$,

7.11-7.35 (5H, m). ${ }^{13} \mathrm{C} \mathrm{NMR}\left(\mathrm{CDCl}_{3}\right) \delta 17.7,25.3,25.7,30.4,51.7,123.8,125.3,128.7$, 132.0, 141.9. EIMS: $m / z$ (rel intensity) $222\left(\mathrm{M}^{+}+1,13\right), 206$ (5), 138 (31), 109 (100), 94 (20), 70 (96), 65 (27), 41 (54). Anal. calcd for $\mathrm{C}_{13} \mathrm{H}_{19} \mathrm{NS}$ : C, 70.54; H, 8.65; N,6.33. Found: C, 70.32; H, 8.41; N, 6.47.

\section{Characterization of Products}

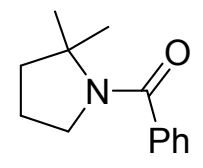

$2 b$

Colorless oil. ${ }^{1} \mathrm{H}$ NMR $\left(300 \mathrm{MHz}, \mathrm{CDCl}_{3}\right) \delta 1.59(6 \mathrm{H}, \mathrm{s}), 1.75-1.88(4 \mathrm{H}, \mathrm{m}), 3.38(2 \mathrm{H}, \mathrm{t}, J$ $=6.3 \mathrm{~Hz}), 7.34-7.39(5 \mathrm{H}, \mathrm{m}) .{ }^{13} \mathrm{C} \mathrm{NMR}\left(\mathrm{CDCl}_{3}\right) \delta 23.1,25.8,42.3,51.6,62.1,126.3$, 128.1, 129.01, 139.1, 169.4. EIMS: $m / z$ (rel intensity) $204\left(\mathrm{M}^{+}+1,2.5\right), 188(21), 106(8)$, 105 (100), 77 (37), 55 (8), 51 (11), 41 (9). HRMS cacld for $\mathrm{C}_{13} \mathrm{H}_{17} \mathrm{NNaO}(\mathrm{M}+\mathrm{Na})$ : 226.1202. Found: 226.1212.

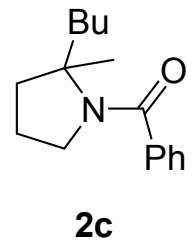

Colorless oil. ${ }^{1} \mathrm{H}$ NMR (300 MHz, $\left.\mathrm{CDCl}_{3}\right) \delta 0.93(3 \mathrm{H}, \mathrm{t}, J=6.9 \mathrm{~Hz}), 1.21-1.40(4 \mathrm{H}, \mathrm{m})$, $1.55(3 \mathrm{H}, \mathrm{s}), 1.66-1.81(3 \mathrm{H}, \mathrm{m}), 1.89-2.20(3 \mathrm{H}, \mathrm{m}), 3.36(2 \mathrm{H}, \mathrm{dd}, J=7.6,5.7 \mathrm{~Hz})$, 7.35-7.43 (5H, m). ${ }^{13} \mathrm{C} \mathrm{NMR}\left(\mathrm{CDCl}_{3}\right) \delta 14.2,23.1,23.3,24.6,26.9,37.5,38.5,52.1,65.1$, 126.3, 128.2, 129.0, 139.2, 169.2. EIMS: $m / z$ (rel intensity) $246\left(\mathrm{M}^{+}+1,10\right), 230(3), 189$ (12), 188 (69), 105 (100), 77 (27), 51 (7), 41 (10). Anal. calcd for $\mathrm{C}_{16} \mathrm{H}_{23} \mathrm{NO}: \mathrm{C}, 78.32 ; \mathrm{H}$, 9.45; N, 5.71. Found: C, 78.34; H, 9.48; N, 5.41. 


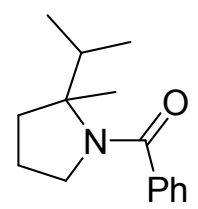

2d

White solid. Mp 63-64 ${ }^{\circ} \mathrm{C} .{ }^{1} \mathrm{H}$ NMR $\left(300 \mathrm{MHz}, \mathrm{CDCl}_{3}\right) \delta 0.94(6 \mathrm{H}, \mathrm{d}, J=6.9 \mathrm{~Hz})$, 1.53-1.57 (1H, m), $1.60(3 \mathrm{H}, \mathrm{s}), 1.71-1.86(2 \mathrm{H}, \mathrm{m}), 1.94-2.04(1 \mathrm{H}, \mathrm{m}), 2.96-3.10(1 \mathrm{H}, \mathrm{m})$, 3.26-3.40 (2H, m), 7.35-7.42 (5H, m). ${ }^{13} \mathrm{C} \mathrm{NMR}\left(\mathrm{CDCl}_{3}\right) \delta 17.0,18.1,23.3,23.4,32.2$, 33.2, 52.8, 68.8, 126.1, 128.2, 129.0, 139.3, 169.0. EIMS: $m / z$ (rel intensity) $232\left(\mathrm{M}^{+}+1\right.$, 1), 188 (34), 106 (8), 105 (100), 95 (3), 77 (30), 51(5), 41 (4). Anal. calcd for $\mathrm{C}_{15} \mathrm{H}_{21} \mathrm{NO}$ : C, 77.88; H, 9.15; N, 6.05. Found: C, 78.02; H, 9.18; N, 5.89.

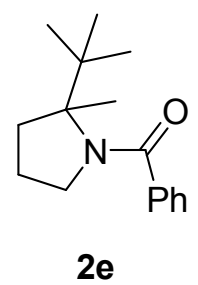

White solid. Mp 78-79 ${ }^{\circ} \mathrm{C} .{ }^{1} \mathrm{H}$ NMR $\left(300 \mathrm{MHz}, \mathrm{CDCl}_{3}\right) \delta 1.12(9 \mathrm{H}, \mathrm{s}), 1.59-1.69(3 \mathrm{H}, \mathrm{m})$, $1.71(3 \mathrm{H}, \mathrm{s}), 2.09-2.22(1 \mathrm{H}, \mathrm{m}), 3.28-3.43(2 \mathrm{H}, \mathrm{m}), 7.33-7.43(5 \mathrm{H}, \mathrm{m}) .{ }^{13} \mathrm{C} \mathrm{NMR}\left(\mathrm{CDCl}_{3}\right)$ $\delta 20.8,24.0,27.2,38.2,40.0,55.1,71.5,126.5,128.2,129.2,140.1,170.9$. EIMS: $m / z$ (rel intensity) 245 ( $\left.\mathrm{M}^{+}, 0.7\right), 230$ (2), 188 (37), 105 (100), 77 (24), 57 (5), 55 (6), 41 (7). Anal. calcd for $\mathrm{C}_{16} \mathrm{H}_{23} \mathrm{NO}$ : C, 78.32; H, 9.45; N, 5.71. Found: C, 78.38; H, 9.21; N, 5.54.

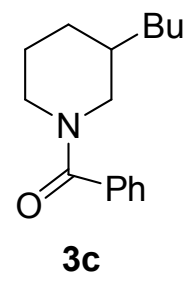

Colorless oil. ${ }^{1} \mathrm{H}$ NMR $\left(300 \mathrm{MHz}, \mathrm{CDCl}_{3}\right) \delta$ 0.83/0.90 (3H, br), 1.13-1.93 $(11 \mathrm{H}, \mathrm{m})$, 2.44-2.68 (1H, m), 2.82-2.93 (1H, m), $3.63(1 \mathrm{H}, \mathrm{br}), 4.50-4.59(1 \mathrm{H}, \mathrm{m}), 7.39(5 \mathrm{H}, \mathrm{s}) .{ }^{13} \mathrm{C}$ 
$\operatorname{NMR}\left(\mathrm{CDCl}_{3}\right) \delta 13.9,22.8,24.8 / 25.9,28.8 / 29.7,31.2,33.0 / 33.6,35.9 / 36.9,43.0 / 48.2$, 48.5/53.7,126.8, 128.3, 129.3, 136.6, 170.2. EIMS: $m / z$ (rel intensity) $245\left(\mathrm{M}^{+}, 21\right), 216$ (6), 202 (5), 188 (43), 105 (100), 77 (45), 51 (9), 41 (12). Anal. calcd for $\mathrm{C}_{16} \mathrm{H}_{23} \mathrm{NO}$ : C, 78.32; H, 9.45; N, 5.71. Found: C, 78.30; H, 9.60; N, 5.62.

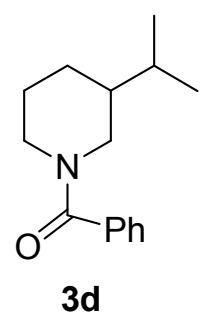

Colorless oil. ${ }^{1} \mathrm{H}$ NMR $\left(300 \mathrm{MHz}, \mathrm{CDCl}_{3}\right) \delta$ 0.72-0.99 (6H, m), 1.20-1.92 (6H, m), 2.48-2.94 (2H, m), 3.66-3.73 (1H, m), $4.66(1 \mathrm{H}, \mathrm{br}), 7.39(5 \mathrm{H}, \mathrm{s}) .{ }^{13} \mathrm{C} \mathrm{NMR}\left(\mathrm{CDCl}_{3}\right) \delta$ $19.5,25.3 / 26.2,27.9,30.4 / 30.8,42.2 / 43.0,43.6 / 46.3,48.5 / 51.9,126.8,128.3,129.3$, 136.5, 170.3. EIMS: $m / z$ (rel intensity) $231\left(\mathrm{M}^{+}, 26\right), 216$ (2), 189 (10), 188 (79), 105 (100), 77 (46), 51 (13), 41 (15). Anal. calcd for $\mathrm{C}_{15} \mathrm{H}_{21} \mathrm{NO}$ : C, 77.88; H, 9.15; N, 6.05. Found: C, 78.09; H, 9.29; N, 5.88 .

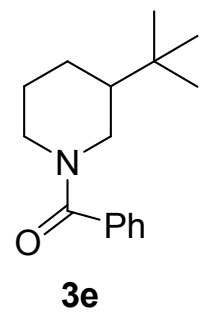

Colorless oil. ${ }^{1} \mathrm{H}$ NMR $\left(300 \mathrm{MHz}, \mathrm{CDCl}_{3}\right) \delta$ 0.75/0.96 (9H, s), 1.17-1.96 (5H, m), 2.38-2.90 (2H, m), 3.68-3.81 (1H, m), 4.71-4.87 (1H, m), $7.39(5 \mathrm{H}, \mathrm{s}) .{ }^{13} \mathrm{C} \mathrm{NMR}\left(\mathrm{CDCl}_{3}\right)$ $\delta$ 25.9, 26.7, 27.2/27.4, 31.8, 42.9/44.4, 46.0/47.4, 48.4/50.2, 126.7, 128.3, 129.3, 136.6, 170.3. EIMS: $m / z$ (rel intensity) $245\left(\mathrm{M}^{+}, 10\right), 230$ (3), 188 (73), 105 (100), 77 (40), 57 (6), 55 (5), 41 (15). HRMS cacld for $\mathrm{C}_{16} \mathrm{H}_{23} \mathrm{NO}$ : 245.1780. Found: 245.1776. 


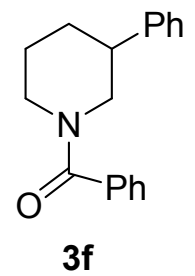

Colorless oil. ${ }^{1} \mathrm{H}$ NMR $\left(300 \mathrm{MHz}, \mathrm{CDCl}_{3}\right) \delta$ 1.66-2.13 (4H, m), $2.86(2 \mathrm{H}, \mathrm{br}), 2.98-3.07$ $(1 \mathrm{H}, \mathrm{m}), 3.83(1 \mathrm{H}, \mathrm{br}), 4.84(1 \mathrm{H}, \mathrm{br}), 7.10-7.41(10 \mathrm{H}, \mathrm{m}) .{ }^{13} \mathrm{C} \mathrm{NMR}\left(\mathrm{CDCl}_{3}\right) \delta 25.4 / 26.3$, $31.9,42.4 / 42.7,43.7 / 54.5,48.2 / 48.8,126.8,127.0,128.5,128.6,129.5,136.3$, 142.7/143.0, 170.4. EIMS: $m / z$ (rel intensity) $265\left(\mathrm{M}^{+}, 28\right), 250$ (11), 131 (11), 105 (100), 91 (15), 77 (40), 51 (11), 42 (7). HRMS cacld for $\mathrm{C}_{18} \mathrm{H}_{19} \mathrm{NO}$ : 165.1467. Found: 265.1463.

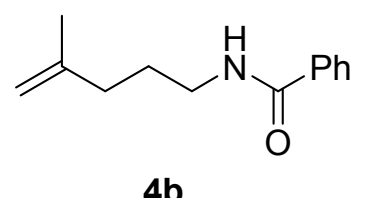

Colorless oil. ${ }^{1} \mathrm{H}$ NMR $\left(300 \mathrm{MHz}, \mathrm{CDCl}_{3}\right) \delta 1.70-1.80(5 \mathrm{H}, \mathrm{m}), 2.09(2 \mathrm{H}, \mathrm{t}, J=7.5 \mathrm{~Hz})$, 3.40-3.46 (2H, m), 4.71-4.74 (2H, m), $6.64(1 \mathrm{H}, \mathrm{br}), 7.36-7.49(3 \mathrm{H}, \mathrm{m}), 7.75-7.79(2 \mathrm{H}, \mathrm{m})$. ${ }^{13} \mathrm{C} \mathrm{NMR}\left(\mathrm{CDCl}_{3}\right) \delta 22.2,27.3,35.1,39.8,110.4,126.8,128.4,131.2,134.7,144.9,167.5$. EIMS: $m / z$ (rel intensity) $204\left(\mathrm{M}^{+}+1,14\right), 134$ (16), 122 (20), 105 (100), 82 (10), 77 (40), 51 (13), 41 (9). Anal. calcd for $\mathrm{C}_{13} \mathrm{H}_{17} \mathrm{NO}$ : C, 76.81; H, 8.43; N, 6.89. Found: $\mathrm{C}, 76.93 ; \mathrm{H}$, $8.46 ; \mathrm{N}, 6.87$.

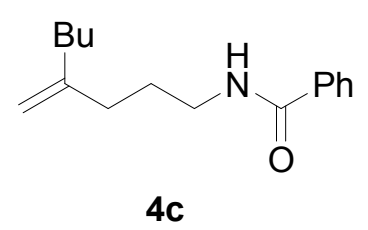

Colorless oil. ${ }^{1} \mathrm{H}$ NMR $\left(300 \mathrm{MHz}, \mathrm{CDCl}_{3}\right) \delta 0.88(3 \mathrm{H}, \mathrm{t}, J=6.9 \mathrm{~Hz}), 1.25-1.44(4 \mathrm{H}, \mathrm{m})$, $1.76(2 \mathrm{H}$, quint, $J=7.5 \mathrm{~Hz}), 2.01(3 \mathrm{H}, \mathrm{t}, J=7.4 \mathrm{~Hz}), 2.11(3 \mathrm{H}, \mathrm{t}, J=7.5 \mathrm{~Hz}), 3.46(2 \mathrm{H}, \mathrm{q}$, $J=6.6 \mathrm{~Hz}), 4.75(2 \mathrm{H}, \mathrm{s}), 6.20(1 \mathrm{H}, \mathrm{br}), 7.38-7.51(3 \mathrm{H}, \mathrm{m}), 7.74(2 \mathrm{H}, \mathrm{d}, J=7.8 \mathrm{~Hz}) .{ }^{13} \mathrm{C}$ NMR $\left(\mathrm{CDCl}_{3}\right) \delta 13.9,22.4,27.6,29.9,33.5,35.6,39.9,109.2,126.8,128.4,131.2,134.8$, 149.1, 167.5. EIMS: $m / z$ (rel intensity) $246\left(\mathrm{M}^{+}+1,31\right), 134$ (37), 124 (19), 122 (37), 105 (100), 95 (15), 77 (35), 41 (17). HRMS cacld for $\mathrm{C}_{16} \mathrm{H}_{23} \mathrm{NO}$ : 245.1780. Found: 245.1779. 


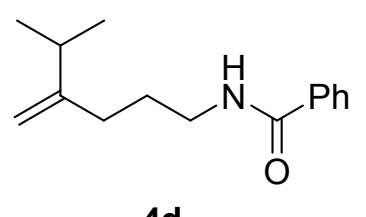

4d

Colorless oil. ${ }^{1} \mathrm{H}$ NMR (300 MHz, $\left.\mathrm{CDCl}_{3}\right) \delta 1.03(6 \mathrm{H}, \mathrm{d}, J=6.9 \mathrm{~Hz}), 1.73-1.83(2 \mathrm{H}, \mathrm{m})$, $2.14(2 \mathrm{H}, \mathrm{t}, J=7.6 \mathrm{~Hz}), 2.18-2.32(1 \mathrm{H}, \mathrm{m}), 3.48(2 \mathrm{H}, \mathrm{q}, J=7.3 \mathrm{~Hz}), 4.73(1 \mathrm{H}, \mathrm{s}), 4.80(1 \mathrm{H}$, s), $6.25(1 \mathrm{H}, \mathrm{br}), 7.40-7.52(3 \mathrm{H}, \mathrm{m}), 7.74-7.78(2 \mathrm{H}, \mathrm{m}) .{ }^{13} \mathrm{C} \mathrm{NMR}\left(\mathrm{CDCl}_{3}\right) \delta 21.8,28.0$, 31.8, 33.7, 40.0, 106.9, 126.8, 128.5, 131.3, 134.8, 155.1, 167.5. EIMS: $m / z$ (rel intensity) $232\left(\mathrm{M}^{+}+1,2.5\right), 147$ (7), 134 (30), 122 (27), 105 (100), 95 (23), 77 (49), 51 (15). Anal. calcd for $\mathrm{C}_{15} \mathrm{H}_{21} \mathrm{NO}$ : C, 77.88; H, 9.15; N, 6.05. Found: C, 78.04; H, 9.28; N, 5.91.

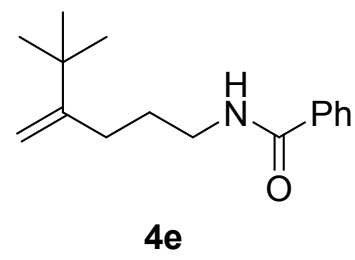

White solid. Mp 66-67 ${ }^{\circ} \mathrm{C} .{ }^{1} \mathrm{H}$ NMR $\left(300 \mathrm{MHz}, \mathrm{CDCl}_{3}\right) \delta 1.06(9 \mathrm{H}, \mathrm{s}), 1.76-1.86(2 \mathrm{H}, \mathrm{m})$, $2.13(2 \mathrm{H}, \mathrm{t}, J=7.8 \mathrm{~Hz}), 3.50(2 \mathrm{H}, \mathrm{q}, J=6.6 \mathrm{~Hz}), 4.73(1 \mathrm{H}, \mathrm{s}), 4.91(1 \mathrm{H}, \mathrm{s}), 6.26(1 \mathrm{H}, \mathrm{br})$, 7.41-7.52 (3H, m), $7.77(2 \mathrm{H}, \mathrm{d}, J=6.9 \mathrm{~Hz}) .{ }^{13} \mathrm{C} \mathrm{NMR}\left(\mathrm{CDCl}_{3}\right) \delta 28.5,29.1,29.3,36.2$, 40.2, 106.3 , 126.9, 128.5, 131.3, 134.8, 157.0, 167.7. EIMS: $m / z$ (rel intensity) $246\left(\mathrm{M}^{+}+1\right.$, 1.5), 147 (6), 134 (19), 124 (32), 105 (100), 77 (38), 57 (7), 41 (13). Anal. calcd for $\mathrm{C}_{16} \mathrm{H}_{23} \mathrm{NO}$ : C, 78.32; H, 9.45; N, 5.72. Found: C, 78.50; H, 9.70; N, 5.58.

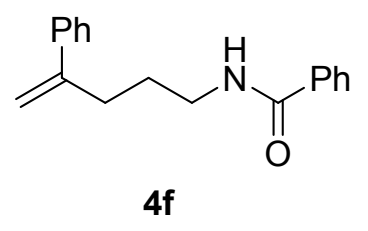

White solid. Mp 63-64 ${ }^{\circ} \mathrm{C} .{ }^{1} \mathrm{H}$ NMR $\left(300 \mathrm{MHz}, \mathrm{CDCl}_{3}\right) \delta 1.79(2 \mathrm{H}$, quint, $J=7.4 \mathrm{~Hz})$, $2.63(2 \mathrm{H}, \mathrm{t}, J=7.4 \mathrm{~Hz}), 3.48(2 \mathrm{H}, \mathrm{q}, J=6.8 \mathrm{~Hz}), 5.12(1 \mathrm{H}, \mathrm{s}), 5.31(1 \mathrm{H}, \mathrm{s}), 6.13(1 \mathrm{H}, \mathrm{br})$, 7.27-7.73 (10H, m). ${ }^{13} \mathrm{C} \mathrm{NMR}\left(\mathrm{CDCl}_{3}\right) \delta 28.1,32.8,39.7,113.0,126.1,126.9,127.6$, 
128.4, 128.5, 131.3, 134.7, 140.8, 147.6, 167.6. EIMS: $m / z$ (rel intensity) $265\left(\mathrm{M}^{+}, 0.7\right)$, 144 (78), 129 (43), 118 (14), 105 (100), 77 (54), 51 (13), 41 (13). Anal. calcd for $\mathrm{C}_{15} \mathrm{H}_{21} \mathrm{NO}$ : C, 81.47; H, 7.22; N, 5.28. Found: C, 81.38; H, 7.27; N, 5.02.

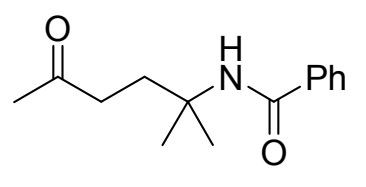

6c

White solid. Mp 84-85 ${ }^{\circ} \mathrm{C} .{ }^{1} \mathrm{H}$ NMR $\left(300 \mathrm{MHz}, \mathrm{CDCl}_{3}\right) \delta 1.39(6 \mathrm{H}, \mathrm{s}), 1.99(2 \mathrm{H}, \mathrm{t}, J=7.0$ $\mathrm{Hz}), 2.11(3 \mathrm{H}, \mathrm{s}), 2.51(2 \mathrm{H}, \mathrm{t}, J=7.0 \mathrm{~Hz}), 6.37(1 \mathrm{H}, \mathrm{br}), 7.32-7.43(3 \mathrm{H}, \mathrm{m}), 7.68(2 \mathrm{H}, \mathrm{d}, J$ $=7.8 \mathrm{~Hz}) .{ }^{13} \mathrm{C} \mathrm{NMR}\left(\mathrm{CDCl}_{3}\right) \delta 26.8,29.9,34.5,39.0,53.6,126.7,128.5,131.1,135.6$, 166.9, 209.6. EIMS: $m / z$ (rel intensity) $218\left(\mathrm{M}^{+}\right.$-15, 0.3), 176 (6), 162 (11), 122 (8), 105 (100), 77 (41), 51 (10), 43 (25). Anal. calcd for $\mathrm{C}_{14} \mathrm{H}_{19} \mathrm{NO}_{2}$ : C, 72.07; H, 8.21; N, 6.00. Found: C, 71.79; H, 8.21; N, 5.89 .

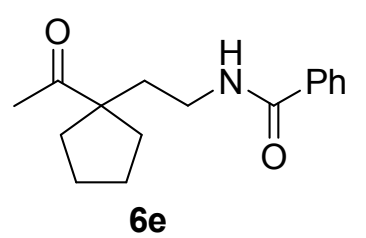

Colorless oil. ${ }^{1} \mathrm{H}$ NMR (300 MHz, $\left.\mathrm{CDCl}_{3}\right) \delta$ 1.52-1.67 (6H, m), 1.98-2.10 (4H, m), 2.19 $(3 \mathrm{H}, \mathrm{s}), 3.37(2 \mathrm{H}, \mathrm{q}, J=6.7 \mathrm{~Hz}), 6.36(1 \mathrm{H}, \mathrm{br}), 7.43-7.50(3 \mathrm{H}, \mathrm{m}), 7.74(2 \mathrm{H}, \mathrm{d}, J=7.2 \mathrm{~Hz})$. ${ }^{13} \mathrm{C}$ NMR $\left(\mathrm{CDCl}_{3}\right) \delta 25.0,25.6,34.8,37.2,37.5,59.0,126.8,128.5,131.4,134.4,167.3$, 213.5. EIMS: $m / z$ (rel intensity) $260\left(\mathrm{M}^{+}+1,1\right), 148$ (13), 134 (14), 122 (11), 105 (100), 77 (37), 67 (6), 43 (14). HRMS cacld for $\mathrm{C}_{16} \mathrm{H}_{21} \mathrm{NO}_{2}$ : 259.1572. Found: 259.1582.

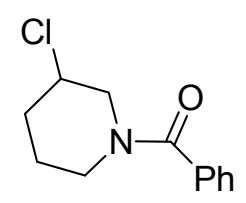

$7 a$

Colorless oil. ${ }^{1} \mathrm{H}$ NMR (300 MHz, $\left.\mathrm{CDCl}_{3}\right) \delta$ 1.49-1.22 (4H, m), 3.22-4.42 (5H, m), 7.41 
(5H, br). ${ }^{13} \mathrm{C} \mathrm{NMR}\left(\mathrm{CDCl}_{3}\right) \delta 22.5 / 24.5,34.3,42.2,47.5 / 49.2,54.5,126.9,128.4,129.7$, 135.6, 170.8. EIMS: $m / z$ (rel intensity) $223\left(\mathrm{M}^{+}, 20\right), 222$ (46), 188 (35), 105 (100), 86 (13), 84 (21), 77 (45), 51 (11). Anal. calcd for $\mathrm{C}_{12} \mathrm{H}_{14}$ ClNO: C, 64.43; H, 6.31; N, 6.26. Found: $\mathrm{C}, 64.31 ; \mathrm{H}, 6.32 ; \mathrm{N}, 6.17$.

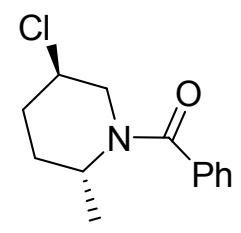

\section{7b-trans}

White solid. Mp 118-119 ${ }^{\circ} \mathrm{C} .{ }^{1} \mathrm{H}$ NMR (300 MHz, $\left.\mathrm{CDCl}_{3}\right) \delta 1.23(3 \mathrm{H}, \mathrm{d}, J=6.9 \mathrm{~Hz}), 1.40$ $(1 \mathrm{H}, \mathrm{d}, J=14.7 \mathrm{~Hz}), 1.93(1 \mathrm{H}, \mathrm{d}, J=14.4 \mathrm{~Hz}), 2.09-2.21(1 \mathrm{H}, \mathrm{m}), 2.27-2.40(1 \mathrm{H}, \mathrm{m}), 3.39$ $(1 \mathrm{H}, \mathrm{d}, J=14.4 \mathrm{~Hz}), 4.16(1 \mathrm{H}, \mathrm{br}), 4.31(1 \mathrm{H}, \mathrm{s}), 4.72(1 \mathrm{H}, \mathrm{br}), 7.40(5 \mathrm{H}, \mathrm{s}) \cdot{ }^{13} \mathrm{C} \mathrm{NMR}$ $\left(\mathrm{CDCl}_{3}\right) \delta$ 15.9, 23.6, 26.9, 44.9/45.5, 45.8/47.2, 56.3, 126.7, 128.4, 129.3, 136.4, 171.5. EIMS: $m / z$ (rel intensity) $237\left(\mathrm{M}^{+}, 4\right), 202$ (6), 106 (8), 105 (100), 77 (32), 55 (3), 51 (8), 41 (3). Anal. calcd for $\mathrm{C}_{13} \mathrm{H}_{16}$ ClNO: $\mathrm{C}, 65.68 ; \mathrm{H}, 6.78 ; \mathrm{N}, 5.89$. Found: $\mathrm{C}, 65.81 ; \mathrm{H}, 6.99$; N, 5.77 .

The cis-isomer (7b-cis) of $\mathbf{7 b}$-trans could not be separated from the uncyclized product $\mathbf{8 b}$ but could be characterized by it reduction to the known compound $\mathbf{7 b}$ ", which could be easily separated from $\mathbf{8 b}$.

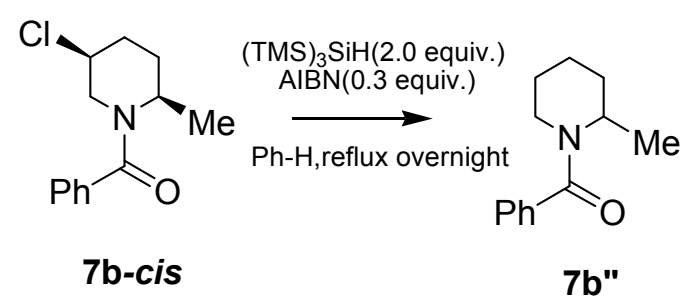




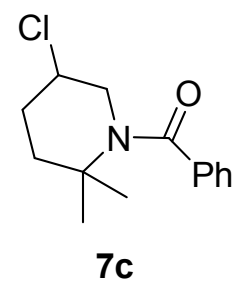

Colorless oil. ${ }^{1} \mathrm{H}$ NMR $\left(300 \mathrm{MHz}, \mathrm{CDCl}_{3}\right) \delta 1.57(6 \mathrm{H}, \mathrm{s}), 1.58-1.65(1 \mathrm{H}, \mathrm{m}), 1.86-1.97$ $(2 \mathrm{H}, \mathrm{m}), 2.13-2.25(1 \mathrm{H}, \mathrm{m}), 3.35(1 \mathrm{H}, \mathrm{dd}, J=14.4,8.4 \mathrm{~Hz}), 3.64(1 \mathrm{H}, \mathrm{dd}, J=14.4,4.2 \mathrm{~Hz})$, 4.07-4.15 (1H, m), 7.36-7.52 (5H, m). ${ }^{13} \mathrm{C}$ NMR $\left(\mathrm{CDCl}_{3}\right) \delta 24.2,25.8,30.1,37.5,51.2$, 54.8, 55.3 127.5, 128.5, 130.1, 137.7, 173.8. EIMS: $m / z$ (rel intensity) $260\left(\mathrm{M}^{+}+1,0.5\right)$, 236 (10), 216 (2), 105 (100), 77 (37), 55 (4), 51 (9), 41 (8). Anal. calcd for $\mathrm{C}_{14} \mathrm{H}_{18} \mathrm{ClNO}$ : C, 66.79; H, 7.21; N, 5.56. Found: C, 66.81; H, 7.16; N, 5.30.

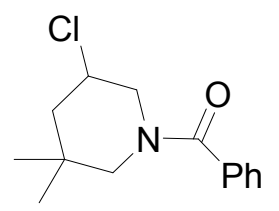

$7 d$

Colorless oil. ${ }^{1} \mathrm{H}$ NMR $\left(300 \mathrm{MHz}, \mathrm{CDCl}_{3}\right) \delta 0.88-1.04(6 \mathrm{H}, \mathrm{m}), 1.59(1 \mathrm{H}, \mathrm{t}, J=12.3 \mathrm{~Hz})$, $2.06(1 \mathrm{H}, \mathrm{d}, J=10.8 \mathrm{~Hz}), 2.52-5.11(5 \mathrm{H}, \mathrm{m}), 7.37-7.46(5 \mathrm{H}, \mathrm{m}) .{ }^{13} \mathrm{C} \mathrm{NMR}\left(\mathrm{CDCl}_{3}\right) \delta$ 23.6/28.2, 34.4, 48.6, 49.5, 51.4/52.1, 54.6/58.0, 126.9, 128.5, 129.8, 135.6, 170.8. EIMS: $m / z$ (rel intensity) $250\left(\mathrm{M}^{+}\right.$- 1, 4), 236 (5), 216 (18), 105 (100), 77 (38), 68 (7), 51 (9), 41 (14). Anal. calcd for $\mathrm{C}_{14} \mathrm{H}_{18} \mathrm{ClNO}$ : C, 66.79; H, 7.21; N, 5.56. Found: C, 66.63; H, 7.36; N, 5.24 .

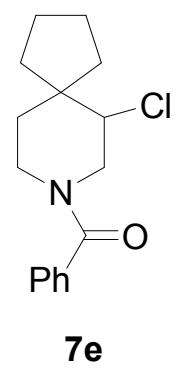

White solid. Mp 59-62 ${ }^{\circ} \mathrm{C} .{ }^{1} \mathrm{H}$ NMR (300 MHz, $\left.\mathrm{CDCl}_{3}\right) \delta$ 1.32-1.92 (10H, m), 3.37-4.07 (5H, m), $7.40(5 \mathrm{H}, \mathrm{s}) .{ }^{13} \mathrm{C} \mathrm{NMR}\left(\mathrm{CDCl}_{3}\right) \delta 24.9,25.3 / 25.6,33.4 / 33.9,35.4 / 36.2,36.7 / 39.2$, 
44.8/46.5, 47.1, 51.8, 65.2/65.9, 127.1, 128.5, 129.7, 135.8, 171.1. EIMS: $m / z$ (rel intensity) 277 (M+, 7), 242 (13), 148 (5), 105 (100), 95 (4), 77 (43), 51 (10), 41 (7). Anal. calcd for $\mathrm{C}_{16} \mathrm{H}_{20} \mathrm{ClNO}$ : C, 69.18; H, 7.26; N, 5.04. Found: C, 69.40; H, 7.33; N, 4.78.

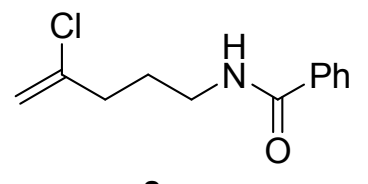

$8 a$

Colorless oil. ${ }^{1} \mathrm{H}$ NMR (300 MHz, $\left.\mathrm{CDCl}_{3}\right) \delta 1.87-1.95(2 \mathrm{H}, \mathrm{m}), 2.44(2 \mathrm{H}, \mathrm{t}, J=7.3 \mathrm{~Hz})$, 3.45-3.51 (2H, q, $J=6.6 \mathrm{~Hz}), 5.19(2 \mathrm{H}, \mathrm{d}, J=1.5 \mathrm{~Hz}), 6.43(1 \mathrm{H}, \mathrm{br}), 7.39-7.52(3 \mathrm{H}, \mathrm{m})$, 7.53-7.78 (2H, m). ${ }^{13} \mathrm{C} \mathrm{NMR}\left(\mathrm{CDCl}_{3}\right) \delta 27.1,36.5,38.9,112.6,126.8,128.4,131.3,134.4$, 141.7, 167.6. EIMS: $m / z$ (rel intensity) $224\left(\mathrm{M}^{+}+1,7\right), 188$ (19), 134 (12) 118 (5), 105 (100), 77 (34), 51 (15), 41 (5). Anal. calcd for $\mathrm{C}_{12} \mathrm{H}_{14}$ ClNO: C, 64.43; H, 6.31; N, 6.26. Found: $\mathrm{C}, 64.01 ; \mathrm{H}, 6.28 ; \mathrm{N}, 6.14$.

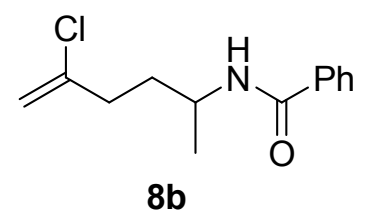

White solid. Mp 99-100 ${ }^{\circ} \mathrm{C} .{ }^{1} \mathrm{H}$ NMR $\left(300 \mathrm{MHz}, \mathrm{CDCl}_{3}\right) \delta 1.28(3 \mathrm{H}, \mathrm{d}, J=6.9 \mathrm{~Hz})$, 1.79-1.87 (2H, m), 2.43-2.49 (2H, m), 4.22-4.31 $(1 \mathrm{H}, \mathrm{m}), 5.16(1 \mathrm{H}, \mathrm{s}), 5.18(1 \mathrm{H}, \mathrm{s}), 5.88$ $(1 \mathrm{H}, \mathrm{d}, J=6.9 \mathrm{~Hz}), 7.41-7.53(3 \mathrm{H}, \mathrm{m}), 7.73-7.76(2 \mathrm{H}, \mathrm{m}) .{ }^{13} \mathrm{C} \mathrm{NMR}\left(\mathrm{CDCl}_{3}\right) \delta 21.0,34.5$, 36.1, 45.1, 112.4, 126.8, 128.4, 131.3, 134.7, 142.1, 166.9. EIMS: $\mathrm{m} / z$ (rel intensity) 238 $\left(\mathrm{M}^{+}+1,1\right), 202$ (7), $148(10), 132(5), 106(6), 105$ (100), 77 (32), 51 (10). Anal. calcd for $\mathrm{C}_{13} \mathrm{H}_{16} \mathrm{ClNO}$ : C, 65.68; H, 6.78; N, 5.89. Found: C, 65.92; H, 6.55; N, 5.89.

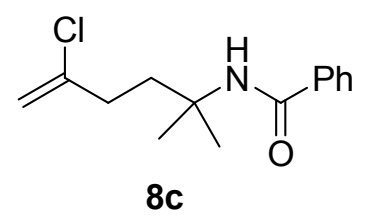

White solid. Mp 84-85 ${ }^{\circ} \mathrm{C} .{ }^{1} \mathrm{H}$ NMR (300 MHz, $\left.\mathrm{CDCl}_{3}\right) \delta 1.39(6 \mathrm{H}, \mathrm{s}), 1.99(2 \mathrm{H}, \mathrm{t}, J=7.0$ 
$\mathrm{Hz}), 2.11(3 \mathrm{H}, \mathrm{s}), 2.51(2 \mathrm{H}, \mathrm{t}, J=7.0 \mathrm{~Hz}), 6.37(1 \mathrm{H}, \mathrm{br}), 7.32-7.43(3 \mathrm{H}, \mathrm{m}), 7.68(2 \mathrm{H}, \mathrm{d}, J$ $=7.8 \mathrm{~Hz}) .{ }^{13} \mathrm{C} \mathrm{NMR}\left(\mathrm{CDCl}_{3}\right) \delta 26.8,29.9,34.5,39.0,53.6,126.7,128.5,131.1,135.6$, 166.9, 209.6. EIMS: $m / z$ (rel intensity) $218\left(\mathrm{M}^{+}-15,0.3\right), 176$ (6), 162 (11), 122 (8), 105 (100), 77 (41), 51 (10), 43 (25). Anal. calcd for $\mathrm{C}_{14} \mathrm{H}_{19} \mathrm{NO}_{2}$ : C, 72.07; H, 8.21; N, 6.00 . Found: C, 71.79; H, 8.21; N, 5.89 .

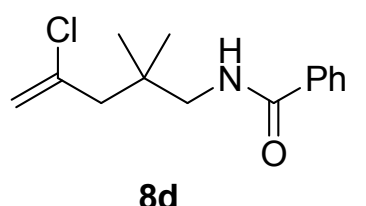

Colorless oil. ${ }^{1} \mathrm{H}$ NMR $\left(300 \mathrm{MHz}, \mathrm{CDCl}_{3}\right) \delta 1.06(6 \mathrm{H}, \mathrm{s}), 2.38(2 \mathrm{H}, \mathrm{s}), 3.42(2 \mathrm{H}, \mathrm{d}, J=6.3$ $\mathrm{Hz}), 5.18(1 \mathrm{H}, \mathrm{s}), 5.35(1 \mathrm{H}, \mathrm{s}), 6.38(1 \mathrm{H}, \mathrm{br}), 7.42-7.51(3 \mathrm{H}, \mathrm{m}), 7.76-7.79(2 \mathrm{H}, \mathrm{m}) .{ }^{13} \mathrm{C}$ NMR $\left(\mathrm{CDCl}_{3}\right) \delta 25.4,35.8,48.8,116.2,126.8,128.5,131.3,134.7,139.0,167.6$. EIMS: $\mathrm{m} / z$ (rel intensity) $252\left(\mathrm{M}^{+}+1,3\right), 216$ (11), 134 (18), 105 (100), 78 (10), 77 (34), 51 (17), 41 (7). ${ }^{13} \mathrm{C}$ NMR $\left(\mathrm{CDCl}_{3}\right) \delta 25.37,35.83,48.77,116.25,126.78,128.50,131.31,134.75$, 139.04, 167.60. Anal. calcd for $\mathrm{C}_{14} \mathrm{H}_{18}$ ClNO: C, 66.79; H, 7.21; N, 5.56. Found: C, 66.80; $\mathrm{H}, 7.18 ; \mathrm{N}, 5.33$.

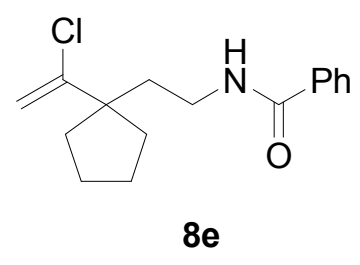

White solid. Mp 77-79 ${ }^{\circ} \mathrm{C} .{ }^{1} \mathrm{H}$ NMR (300 MHz, $\left.\mathrm{CDCl}_{3}\right) \delta$ 1.59-1.73 (6H, m), 1.89-2.00 (4H, m), 3.38-3.45 (2H, m), $5.24(1 \mathrm{H}, \mathrm{d}, J=1.3 \mathrm{~Hz}), 5.29(1 \mathrm{H}, \mathrm{d}, J=1.3 \mathrm{~Hz}), 6.19(1 \mathrm{H}$, br), 7.40-7.49 (3H, m), $7.75(2 \mathrm{H}, \mathrm{d}, J=1.3 \mathrm{~Hz}) .{ }^{13} \mathrm{C} \mathrm{NMR}\left(\mathrm{CDCl}_{3}\right) \delta 23.1,36.6,37.0,37.1$, 53.4, 112.1, 126.8, 128.3, 131.1, 134.6, 148.5, 167.3. EIMS: $m / z$ (rel intensity) $242\left(\mathrm{M}^{+}\right.$$\left.\mathrm{Cl}^{35}, 5\right), 172$ (8), 148 (4), 134 (12), 105 (100), 91 (4), 77 (40), 51 (9). Anal. calcd for $\mathrm{C}_{16} \mathrm{H}_{20} \mathrm{ClNO}: \mathrm{C}, 69.18 ; \mathrm{H}, 7.26 ; \mathrm{N}, 5.04$. Found: $\mathrm{C}, 69.28 ; \mathrm{H}, 7.28 ; \mathrm{N}, 4.90$. 


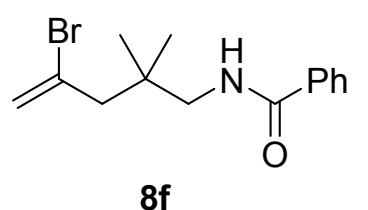

White solid. Mp 61-62 ${ }^{\circ} \mathrm{C} .{ }^{1} \mathrm{H}$ NMR (300 MHz, $\left.\mathrm{CDCl}_{3}\right) \delta 1.06(6 \mathrm{H}, \mathrm{s}), 2.52(2 \mathrm{H}, \mathrm{s}), 3.43$ $(2 \mathrm{H}, \mathrm{d}, J=6.9 \mathrm{~Hz}), 5.62(2 \mathrm{H}, \mathrm{s}), 6.45(1 \mathrm{H}, \mathrm{br}), 7.43-7.52(3 \mathrm{H}, \mathrm{m}), 7.77-7.80(2 \mathrm{H}, \mathrm{m}) .{ }^{13} \mathrm{C}$ NMR $\left(\mathrm{CDCl}_{3}\right) \delta 25.5,36.3,48.6,50.7,121.2,126.9,128.6,129.3,131.5,134.8,167.7$. EIMS: $m / z$ (rel intensity) $216\left(\mathrm{M}^{+}\right.$- Br, 29), 165 (4), 134 (17), 117 (5), 105 (100), 77 (32), 51 (8), 45 (8). HRMS cacld for $\mathrm{C}_{14} \mathrm{H}_{18} \mathrm{NO}$ (M-Br): 216.1388. Found: 216.1381. Anal. calcd for $\mathrm{C}_{14} \mathrm{H}_{18} \mathrm{BrNO}$ : C, 56.77; H, 6.13; N, 4.73. Found: C, 56.70; H, 6.23; N, 4.54.

The 5-exo cyclization product $\mathbf{1 0 a}$ was converted to $\mathbf{1 0 a}$ ' in one step quantitatively, which is a known compound.
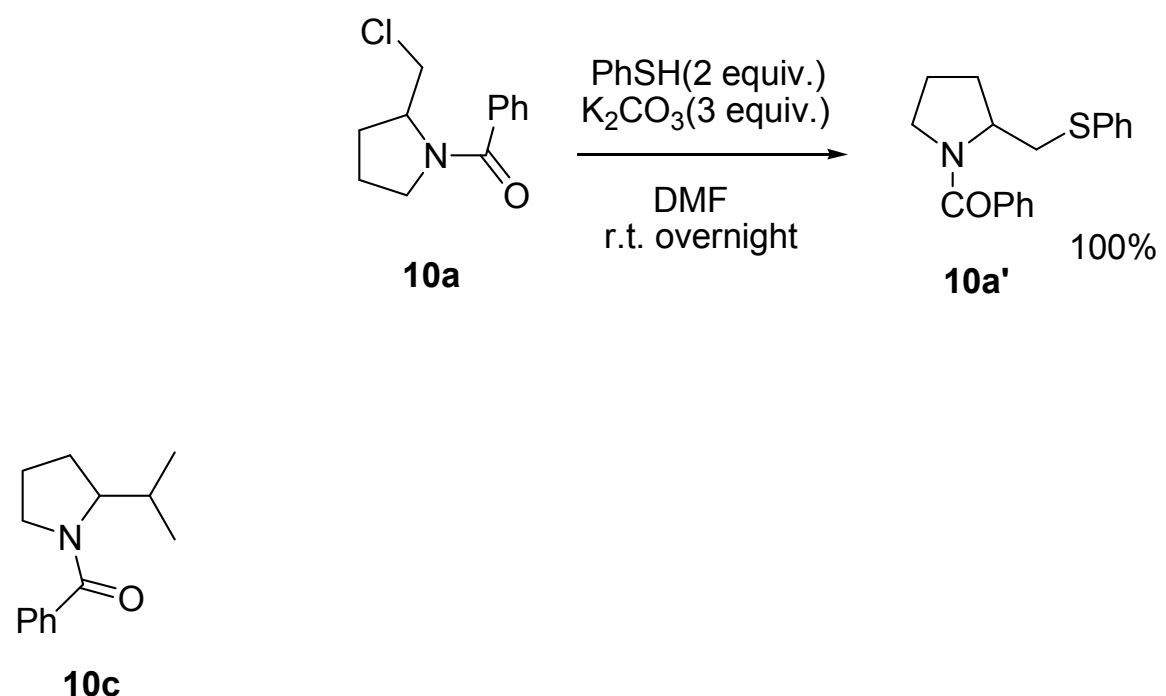

Colorless oil. ${ }^{1} \mathrm{H}$ NMR $\left(300 \mathrm{MHz}, \mathrm{CDCl}_{3}\right) \delta 0.91(3 \mathrm{H}, \mathrm{d}, J=6.6 \mathrm{~Hz}), 0.96(3 \mathrm{H}, \mathrm{d}, J=7.2$ $\mathrm{Hz}), 1.62-1.97(4 \mathrm{H}, \mathrm{m}), 2.42-2.53(1 \mathrm{H}, \mathrm{m}), 3.34-3.49(2 \mathrm{H}, \mathrm{m}), 4.27(1 \mathrm{H}, \mathrm{q}, J=6.8 \mathrm{~Hz})$, 7.36-7.54 (5H, m). ${ }^{13} \mathrm{C}$ NMR $\left(\mathrm{CDCl}_{3}\right) \delta 16.3,19.3,25.2,25.3,29.2,51.2,61.9,127.5$, 128.2, 129.9, 137.6, 170.3. EIMS: $m / z$ (rel intensity) $218\left(\mathrm{M}^{+}+1,3\right), 174$ (30), 134 (2), 106 (9), 105 (100), 77 (24), 51 (5), 41 (6). HRMS cacld for $\mathrm{C}_{14} \mathrm{H}_{19} \mathrm{NO}$ : 217.1467. Found: 217.1458 . 


\section{References for known compounds}

\begin{tabular}{|c|c|}
\hline $2 \mathbf{a}$ & Newcomb, M.; Weber, K. A. J. Org. Chem. 1991, 56, $1309 .$. \\
\hline $3 \mathbf{a}$ & Kazuaki, I.; Takayuki, Y. Org. Lett. 2004, 6, 1983. \\
\hline 3b & Binh, T. N.; Frank, K. C. J. Org. Chem. 1986, 51, $2206 .$. \\
\hline $4 \mathbf{a}$ & $\begin{array}{l}\text { Albert, P.; David, J. A.; Alan, T. P.; David, W. M. Tetrahedron 1996, 52, } \\
3247 .\end{array}$ \\
\hline 6a & $\begin{array}{l}\text { Gyoonhee, H.; Matthew, G.; Mathias, C. M.; Steven, M. W. J. Org. Chem. } \\
\text { 1996, 61, 9483. }\end{array}$ \\
\hline 6b & $\begin{array}{l}\text { Johnson, R. A.; Murray, H. C.; Reineke, L. M. J. Am. Chem. Soc. 1971, 93, } \\
4872 .\end{array}$ \\
\hline 6d & Sheiman, B. M.; et al. J. Org. Chem.USSR (Engl. Transl.), 1969, 5, 1999. \\
\hline $7 \mathbf{b}^{\prime \prime}$ & Jego, J. M.; Carboni, B.; Vaultier, M. Bull. Soc. Chim. 1992, 6, 554. \\
\hline $10 \mathbf{a}^{\prime}$ & $\begin{array}{l}\text { Zakaria K. M. Abd EI, S.; Mohamed I. Al, A.; John, M. M. J. Chem. Soc., } \\
\text { Perkin Trans. } 1 \text { 1988, 2571. }\end{array}$ \\
\hline
\end{tabular}

\section{Computational results on the cyclization of primary aminyl radicals}

Table 1. Calculated free energies at UB3LYP/6-311++G**//UB3LYP/6-31G* level 


\begin{tabular}{ccccc}
\hline \multirow{2}{*}{ entry } & $\mathrm{R}$ & \multicolumn{3}{c}{$\mathrm{G}$ (in hartree) } \\
\cline { 3 - 5 } & & radical A & $\mathrm{TS}_{\text {-exo }}$ & $\mathrm{TS}_{\text {-endo }}$ \\
\hline 1 & $\mathrm{R}=\mathrm{H}$ & -251.184035 & -251.167859 & -251.164747 \\
2 & $\mathrm{R}=\mathrm{Me}$ & -290.485992 & -290.468499 & -290.467896 \\
3 & $\mathrm{R}=\mathrm{Bu}$ & -408.377995 & -408.361105 & -408.360997 \\
4 & $\mathrm{R}=\mathrm{i}-\mathrm{Pr}$ & -369.078391 & -369.061192 & -369.061077 \\
5 & $\mathrm{R}=\mathrm{t}-\mathrm{Bu}$ & -408.371365 & -408.353288 & -408.354641 \\
6 & $\mathrm{R}=\mathrm{Ph}$ & -482.219705 & -482.198685 & -482.204776 \\
7 & $\mathrm{R}=\mathrm{Cl}$ & -710.820956 & -710.799981 & -710.803595 \\
8 & $\mathrm{R}=\mathrm{Br}$ & -2824.742020 & -2824.720559 & -2824.724635
\end{tabular}

For $N$-butylpent-4-enylaminyl radical, $\mathrm{G}=-408.381246$ hartree; $\mathrm{G}_{\mathrm{TS}(5 \text {-exo) }}=-408.354970$.

Table 2. Coordinates of radicals A and transition states at B3LYP/6-31G* level

\section{Radical A $(\mathbf{R}=\mathbf{H})$}

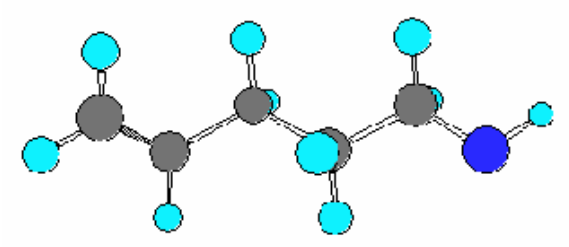

\#p UB3LYP/6-31 g* opt freq=noraman

calculate for Radical A $(\mathrm{R}=\mathrm{H}) \quad \mathrm{HF}=-251.2123245$

$0 \quad 2$

$\mathrm{C}$

$\begin{array}{lll}\text { C } & 1 & 1.33332791\end{array}$

$\begin{array}{lllll}\text { C } & 2 & 1.50461600 & 1 & 125.26624871\end{array}$

$\begin{array}{lllllll}\mathrm{C} & 3 & 1.53916568 & 2 & 112.83557025 & 1 & 119.24332194\end{array}$

$\begin{array}{lllllll}\text { C } & 4 & 1.52981236 & 3 & 112.92484332 & 2 & 178.22259923\end{array}$

$\begin{array}{lllllll}\mathrm{H} & 1 & 1.08677815 & 2 & 121.86156807 & 3 & -179.50433276\end{array}$

$\begin{array}{lllllll}\mathrm{H} & 1 & 1.08851255 & 2 & 121.65337467 & 3 & 0.65103965\end{array}$ 


$\begin{array}{llllllr}\mathrm{H} & 2 & 1.09172187 & 1 & 119.05566792 & 3 & 179.14181704 \\ \mathrm{H} & 3 & 1.10181553 & 2 & 109.25804949 & 1 & -119.76699971 \\ \mathrm{H} & 3 & 1.09863795 & 2 & 109.54417277 & 1 & -3.52401132 \\ \mathrm{H} & 4 & 1.09676619 & 3 & 109.79215835 & 2 & -60.01823246 \\ \mathrm{H} & 4 & 1.09789926 & 3 & 110.05411809 & 2 & 56.76689532 \\ \mathrm{H} & 5 & 1.10776981 & 4 & 110.14479486 & 3 & -56.95704738 \\ \mathrm{H} & 5 & 1.10742871 & 4 & 110.18813349 & 3 & 57.91023142 \\ \mathrm{~N} & 5 & 1.44530357 & 4 & 111.74144453 & 3 & -179.38529460 \\ \mathrm{H} & 5 & 1.03215252 & 5 & 106.34008937 & 4 & -179.32656587\end{array}$

\section{$\operatorname{TS}(5-\operatorname{exo})(\mathbf{R}=\mathbf{H})$}

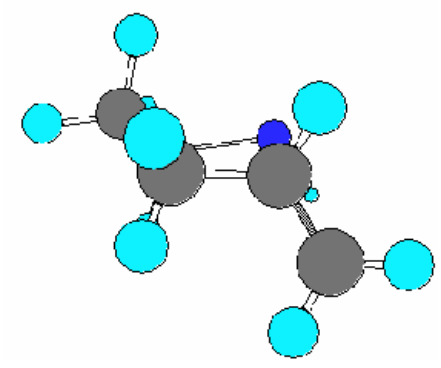

\#p UB3LYP/6-31 g* opt=(ts,z-matrix,noeigentest,nrscale) freq=noraman calculate for TS (5-exo) $(\mathrm{R}=\mathrm{H}) \quad \mathrm{HF}=-251.2016668$
$\begin{array}{ll}0 & 2\end{array}$
$\mathrm{C}$
C $\quad 1 \quad 1.53105098$
$\begin{array}{lllll}\text { C } & 2 & 1.53729737 & 1 & 105.54678809\end{array}$
$\begin{array}{lllllll}\mathrm{C} & 3 & 1.51860178 & 2 & 110.55924971 & 1 & 44.16110411\end{array}$
$\begin{array}{lllllll}\mathrm{C} & 4 & 1.37740788 & 3 & 121.46601907 & 2 & -129.39331370\end{array}$
$\begin{array}{lllllll}\mathrm{H} & 1 & 1.10285514 & 2 & 110.05350242 & 3 & 70.35027395\end{array}$
$\begin{array}{lllllll}\mathrm{H} & 1 & 1.10259928 & 2 & 110.84508517 & 3 & -171.53253701\end{array}$
$\begin{array}{lllllll}\mathrm{H} & 2 & 1.09637594 & 1 & 112.54841467 & 3 & -122.45340668\end{array}$
$\begin{array}{lllllll}\mathrm{H} & 2 & 1.09691985 & 1 & 108.80472005 & 3 & 118.06858646\end{array}$
$\begin{array}{lllllll}\mathrm{H} & 3 & 1.09804563 & 2 & 109.56832361 & 1 & -76.00435758\end{array}$
$\begin{array}{lllllll}\mathrm{H} & 3 & 1.09785922 & 2 & 111.02901821 & 1 & 166.16676279\end{array}$
$\begin{array}{lllllll}\mathrm{H} & 4 & 1.08630481 & 3 & 116.10701798 & 2 & 75.03935487\end{array}$
$\begin{array}{lllllll}\mathrm{H} & 5 & 1.08746686 & 4 & 121.22338684 & 3 & 15.64503056\end{array}$ 


$\begin{array}{rrrrrrr}\mathrm{H} & 5 & 1.08616810 & 4 & 121.61364458 & 3 & -169.48091341 \\ \mathrm{~N} & 1 & 1.45663072 & 2 & 105.50668471 & 3 & -53.98487102 \\ \mathrm{H} & 15 & 1.02712899 & 1 & 109.36804375 & 2 & 143.74974014\end{array}$

\section{TS (6-endo) $(\mathbf{R}=\mathbf{H})$}

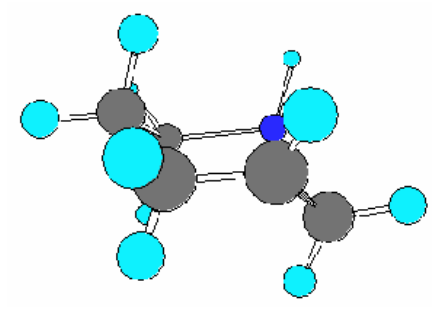

\#p UB3LYP/6-31 g* opt=(ts,z-matrix,noeigentest,nrscale) freq=noraman calculate for TS (6-endo) $(\mathrm{R}=\mathrm{H}) \quad \mathrm{HF}=-251.2000972$

$\begin{array}{crrrrrr}0 & 2 & & & & \\ \mathrm{C} & & & & & \\ \mathrm{C} & 1 & 1.54442755 & & & & \\ \mathrm{C} & 2 & 1.55021045 & 1 & 111.51737817 & & \\ \mathrm{C} & 3 & 1.50243581 & 2 & 109.25647374 & 1 & 54.79988203 \\ \mathrm{C} & 4 & 1.36543703 & 3 & 120.09665598 & 2 & -72.59473163 \\ \mathrm{H} & 1 & 1.09972767 & 2 & 109.37598318 & 3 & 63.01377445 \\ \mathrm{H} & 1 & 1.10485090 & 2 & 108.33154233 & 3 & 178.13049931 \\ \mathrm{H} & 2 & 1.09949923 & 1 & 109.82204758 & 3 & -122.45578300 \\ \mathrm{H} & 2 & 1.09952630 & 1 & 109.04889656 & 3 & 120.81675286 \\ \mathrm{H} & 3 & 1.09911905 & 2 & 108.50117303 & 1 & -65.49986646 \\ \mathrm{H} & 3 & 1.09672189 & 2 & 109.80931764 & 1 & 177.64763742 \\ \mathrm{H} & 4 & 1.08942129 & 3 & 117.95568970 & 2 & 89.52973877 \\ \mathrm{H} & 5 & 1.08624722 & 4 & 120.83664423 & 3 & -27.02934921 \\ \mathrm{H} & 5 & 1.08606543 & 4 & 121.44528639 & 3 & 172.07086019 \\ \mathrm{~N} & 1 & 1.45853284 & 2 & 116.97306542 & 3 & -60.00052395 \\ \mathrm{H} & 15 & 1.03027219 & 1 & 106.64597129 & 2 & -48.49957960\end{array}$

\section{Radical A $(\mathbf{R}=\mathbf{M e})$}




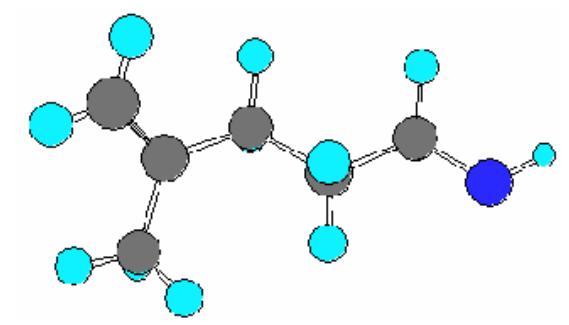

\#p UB3LYP/6-31g* opt freq=noraman

calculate for Radical A $(\mathrm{R}=\mathrm{Me}) \quad \mathrm{HF}=-290.5308563$

02

C

$\begin{array}{lrrrrrr}\mathrm{C} & 1 & 1.33702850 & & & \\ \mathrm{C} & 2 & 1.51504980 & 1 & 121.41126492 & & \\ \mathrm{C} & 3 & 1.54035129 & 2 & 114.08735151 & 1 & 113.87645161 \\ \mathrm{C} & 4 & 1.52985391 & 3 & 112.61960034 & 2 & -178.11558066 \\ \mathrm{H} & 1 & 1.08745311 & 2 & 121.79538112 & 3 & -179.96669023 \\ \mathrm{H} & 1 & 1.08753208 & 2 & 121.76235769 & 3 & 0.04507083 \\ \mathrm{H} & 3 & 1.10194580 & 2 & 109.17135392 & 1 & -124.43568505 \\ \mathrm{H} & 3 & 1.09787094 & 2 & 109.18450713 & 1 & -8.45596438 \\ \mathrm{H} & 4 & 1.09704771 & 3 & 109.70526039 & 2 & -56.72347446 \\ \mathrm{H} & 4 & 1.09660757 & 3 & 110.70549486 & 2 & 60.58893593 \\ \mathrm{H} & 5 & 1.10790100 & 4 & 110.14288277 & 3 & -56.67202392 \\ \mathrm{H} & 5 & 1.10735452 & 4 & 110.23400400 & 3 & 58.19184351 \\ \mathrm{~N} & 5 & 1.44534731 & 4 & 111.73520602 & 3 & -178.97968654 \\ \mathrm{H} & 14 & 1.03216465 & 5 & 106.33316095 & 4 & -179.02669681 \\ \mathrm{C} & 2 & 1.50990214 & 1 & 121.74851089 & 3 & -179.62807061 \\ \mathrm{H} & 16 & 1.09768356 & 2 & 111.66830527 & 1 & -118.58003071 \\ \mathrm{H} & 16 & 1.09423655 & 2 & 111.54382389 & 1 & 2.16566883 \\ \mathrm{H} & 16 & 1.09902771 & 2 & 110.97372498 & 1 & 122.74094116\end{array}$

TS (5-exo) $(\mathrm{R}=\mathrm{Me})$ 


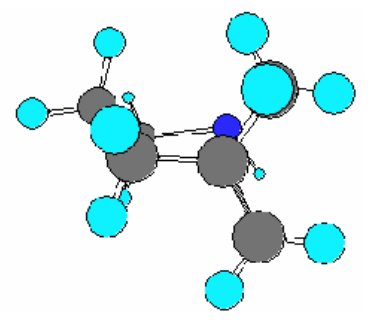

\#p UB3LYP/6-31 g* opt=(ts,z-matrix,noeigentest,nrscale) freq=noraman calculate for TS (5-exo) $(\mathrm{R}=\mathrm{Me}) \quad \mathrm{HF}=-290.5183285$

02

C

$\begin{array}{rrrrrrr}\mathrm{C} & 1 & 1.53042891 & & & \\ \mathrm{C} & 2 & 1.53829658 & 1 & 105.63647863 & & \\ \mathrm{C} & 3 & 1.52811886 & 2 & 111.12005293 & 1 & 44.14430426 \\ \mathrm{C} & 4 & 1.38201777 & 3 & 118.73462334 & 2 & -123.59167331 \\ \mathrm{H} & 1 & 1.10302408 & 2 & 110.08278068 & 3 & 70.53760137 \\ \mathrm{H} & 1 & 1.10280858 & 2 & 110.92548948 & 3 & -171.41586914 \\ \mathrm{H} & 2 & 1.09639635 & 1 & 112.52169343 & 3 & -122.15519026 \\ \mathrm{H} & 2 & 1.09665383 & 1 & 108.95418807 & 3 & 118.56550096 \\ \mathrm{H} & 3 & 1.09717372 & 2 & 109.12338070 & 1 & -76.31933730 \\ \mathrm{H} & 3 & 1.09801844 & 2 & 110.90405989 & 1 & 166.20451204 \\ \mathrm{H} & 5 & 1.08659601 & 4 & 121.47281892 & 3 & 14.31954671 \\ \mathrm{H} & 5 & 1.08693472 & 4 & 121.33744806 & 3 & -170.61766805 \\ \mathrm{~N} & 1 & 1.45553007 & 2 & 105.35502362 & 3 & -53.60860654 \\ \mathrm{H} & 14 & 1.02700208 & 1 & 109.56057483 & 2 & 145.47224594 \\ \mathrm{C} & 4 & 1.51610624 & 3 & 116.58356045 & 2 & 82.39878100 \\ \mathrm{H} & 16 & 1.09895349 & 4 & 109.93652659 & 3 & 71.59491916 \\ \mathrm{H} & 16 & 1.09401851 & 4 & 111.03983034 & 3 & -168.85642178 \\ \mathrm{H} & 16 & 1.09257703 & 4 & 111.49404708 & 3 & -48.66829434\end{array}$

TS (6-endo) $(\mathrm{R}=\mathrm{Me})$ 


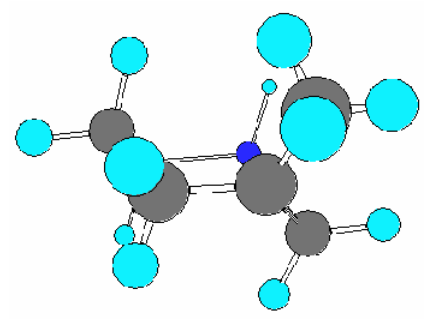

\#p UB3LYP/6-31 g* opt=(ts,z-matrix,noeigentest,nrscale) freq=noraman calculate for TS (6-endo) $(\mathrm{R}=\mathrm{Me}) \quad \mathrm{HF}=-290.5192566$

$\begin{array}{crrrrrr}\text { O } & 2 & & & & & \\ \mathrm{C} & & & & & \\ \mathrm{C} & 1 & 1.54415357 & & & & \\ \mathrm{C} & 2 & 1.54928817 & 1 & 111.92789683 & & \\ \mathrm{C} & 3 & 1.50995680 & 2 & 109.55918248 & 1 & 55.39139061 \\ \mathrm{C} & 4 & 1.36875596 & 3 & 117.60825996 & 2 & -72.55464445 \\ \mathrm{H} & 1 & 1.10006065 & 2 & 109.27576580 & 3 & 63.35670207 \\ \mathrm{H} & 1 & 1.10478742 & 2 & 108.30664881 & 3 & 178.36951776 \\ \mathrm{H} & 2 & 1.09929378 & 1 & 109.78379426 & 3 & -122.52129344 \\ \mathrm{H} & 2 & 1.09993973 & 1 & 109.01700379 & 3 & 121.04124205 \\ \mathrm{H} & 3 & 1.09870682 & 2 & 108.51024455 & 1 & -65.19391786 \\ \mathrm{H} & 3 & 1.09749596 & 2 & 109.66556057 & 1 & 178.10860654 \\ \mathrm{H} & 5 & 1.08544289 & 4 & 120.86940017 & 3 & -26.34627642 \\ \mathrm{H} & 5 & 1.08670437 & 4 & 121.18829343 & 3 & 173.46459341 \\ \mathrm{~N} & 1 & 1.45898308 & 2 & 117.01917390 & 3 & -59.54130137 \\ \mathrm{H} & 14 & 1.03016902 & 1 & 106.60351557 & 2 & -48.81337659 \\ \mathrm{C} & 4 & 1.50429870 & 3 & 118.23075645 & 2 & 94.09369566 \\ \mathrm{H} & 16 & 1.09981708 & 4 & 110.76419210 & 3 & 68.80365260 \\ \mathrm{H} & 16 & 1.09496831 & 4 & 111.90681603 & 3 & -170.95435632 \\ \mathrm{H} & 16 & 1.10048402 & 4 & 112.11283822 & 3 & -49.44642522\end{array}$

Radical A $(\mathbf{R}=\mathbf{B u})$ 


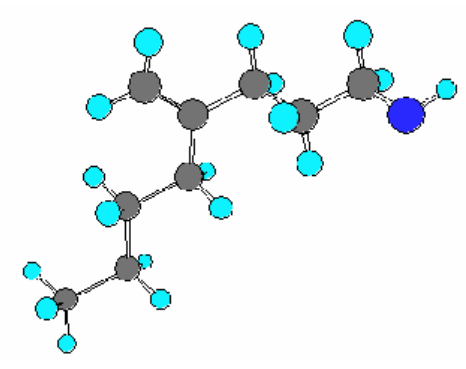

\#p UB3LYP/6-31 g* opt freq=noraman

calculate for Radical A $(\mathrm{R}=\mathrm{Bu}) \quad \mathrm{HF}=-408.4704313$

\begin{tabular}{|c|c|c|c|c|c|c|}
\hline $\mathrm{C}$ & 1 & 1.33743933 & & & & \\
\hline $\mathrm{C}$ & 2 & 1.51751510 & 1 & 120.65003578 & & \\
\hline $\mathrm{C}$ & 3 & 1.54069935 & 2 & 114.14794243 & 1 & 109.57393416 \\
\hline $\mathrm{C}$ & 4 & 1.53000478 & 3 & 112.58921985 & 2 & -178.13062414 \\
\hline $\mathrm{H}$ & 1 & 1.08545953 & 2 & 122.67921144 & 3 & -179.44023993 \\
\hline $\mathrm{H}$ & 1 & 1.08752902 & 2 & 121.19090310 & 3 & 0.49679516 \\
\hline $\mathrm{H}$ & 3 & 1.10162579 & 2 & 109.34495453 & 1 & -128.45899088 \\
\hline $\mathrm{H}$ & 3 & 1.09785783 & 2 & 109.06711468 & 1 & -12.47315658 \\
\hline $\mathrm{H}$ & 4 & 1.09696807 & 3 & 109.64470681 & 2 & -56.78197940 \\
\hline $\mathrm{H}$ & 4 & 1.09654333 & 3 & 110.79371324 & 2 & 60.55216359 \\
\hline $\mathrm{H}$ & 5 & 1.10788208 & 4 & 110.13143271 & 3 & -56.70047923 \\
\hline $\mathrm{H}$ & 5 & 1.10736838 & 4 & 110.20738434 & 3 & 58.14783801 \\
\hline $\mathrm{N}$ & 5 & 1.44537630 & 4 & 111.75728332 & 3 & -179.00595919 \\
\hline $\mathrm{H}$ & 14 & 1.03218463 & 5 & 106.32447041 & 4 & -178.96797638 \\
\hline $\mathrm{C}$ & 2 & 1.51678502 & 1 & 123.59524471 & 3 & -179.84422033 \\
\hline $\mathrm{C}$ & 16 & 1.53205919 & 2 & 116.95708413 & 1 & 1.31866820 \\
\hline $\mathrm{H}$ & 16 & 1.10076575 & 2 & 108.76216979 & 1 & -122.31439842 \\
\hline $\mathrm{H}$ & 16 & 1.10232422 & 2 & 107.98735115 & 1 & 124.64614151 \\
\hline $\mathrm{C}$ & 17 & 1.53436329 & 16 & 112.81690682 & 2 & 179.22789860 \\
\hline $\mathrm{H}$ & 17 & 1.09887709 & 16 & 109.53062835 & 2 & -58.91200725 \\
\hline $\mathrm{H}$ & 17 & 1.09888725 & 16 & 109.54532494 & 2 & 57.30938578 \\
\hline $\mathrm{C}$ & 20 & 1.53199484 & 17 & 113.10672336 & 16 & 179.91464251 \\
\hline $\mathrm{H}$ & 20 & 1.09945894 & 17 & 109.28247623 & 16 & -57.91506344 \\
\hline $\mathrm{H}$ & 20 & 1.09934074 & 17 & 109.30223144 & 16 & 57.71366495 \\
\hline $\mathrm{H}$ & 23 & 1.09598291 & 20 & 111.40883641 & 17 & 179.86445955 \\
\hline
\end{tabular}




$$
\begin{array}{lllllll}
\mathrm{H} & 23 & 1.09696252 & 20 & 111.18571452 & 17 & -60.02186031 \\
\mathrm{H} & 23 & 1.09702591 & 20 & 111.18809907 & 17 & 59.73882750
\end{array}
$$

\section{TS (5-exo) $(\mathrm{R}=\mathrm{Bu})$}

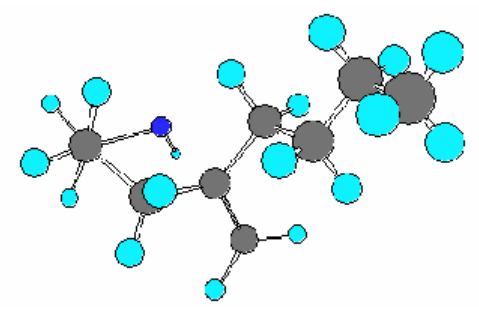

\#p UB3LYP/6-31 g* opt=(ts,z-matrix,noeigentest,nrscale) freq=noraman

calculate for $\mathrm{TS}(5$-exo $)(\mathrm{R}=\mathrm{Bu}) \quad \mathrm{HF}=-408.4588703$

$\begin{array}{lrrrrrr}0 & 2 & & & & & \\ \mathrm{C} & & & & & & \\ \mathrm{C} & 1 & 1.53037955 & & & & \\ \mathrm{C} & 2 & 1.53845286 & 1 & 105.68518675 & & \\ \mathrm{C} & 3 & 1.52904436 & 2 & 111.15511420 & 1 & 43.62745706 \\ \mathrm{C} & 4 & 1.38279547 & 3 & 118.63278902 & 2 & -122.67372741 \\ \mathrm{H} & 1 & 1.10295302 & 2 & 110.14585442 & 3 & 70.47198317 \\ \mathrm{H} & 1 & 1.10279827 & 2 & 110.90623690 & 3 & -171.43799815 \\ \mathrm{H} & 2 & 1.09641428 & 1 & 112.57122165 & 3 & -122.19289621 \\ \mathrm{H} & 2 & 1.09659674 & 1 & 108.87861452 & 3 & 118.52370666 \\ \mathrm{H} & 3 & 1.09713337 & 2 & 109.12268876 & 1 & -76.81818076 \\ \mathrm{H} & 3 & 1.09746702 & 2 & 110.76890196 & 1 & 165.99719643 \\ \mathrm{H} & 5 & 1.08659113 & 4 & 121.62403519 & 3 & 13.81422510 \\ \mathrm{H} & 5 & 1.08728946 & 4 & 121.28224003 & 3 & -171.26916015 \\ \mathrm{~N} & 1 & 1.45523597 & 2 & 105.30284767 & 3 & -53.68906081 \\ \mathrm{H} & 14 & 1.02703295 & 1 & 109.57529789 & 2 & 145.58872766 \\ \mathrm{C} & 4 & 1.52256542 & 3 & 117.19879320 & 2 & 82.85710400 \\ \mathrm{C} & 16 & 1.54015459 & 4 & 113.28638750 & 3 & 79.27573686 \\ \mathrm{H} & 16 & 1.09595664 & 4 & 109.46501520 & 3 & -43.52781992 \\ \mathrm{H} & 16 & 1.09663303 & 4 & 108.77601107 & 3 & -158.89457664 \\ \mathrm{C} & 17 & 1.53431552 & 16 & 112.83442800 & 4 & -179.14559296 \\ \mathrm{H} & 17 & 1.09991055 & 16 & 109.91338231 & 4 & -57.21756300\end{array}$




$\begin{array}{lrrrrrr}\mathrm{H} & 17 & 1.09887686 & 16 & 109.33095455 & 4 & 58.73708623 \\ \mathrm{C} & 20 & 1.53209891 & 17 & 113.17865458 & 16 & -179.89556881 \\ \mathrm{H} & 20 & 1.09936095 & 17 & 109.18724167 & 16 & -57.67094236 \\ \mathrm{H} & 20 & 1.09940481 & 17 & 109.26327466 & 16 & 57.83151354 \\ \mathrm{H} & 23 & 1.09618964 & 20 & 111.43046300 & 17 & 179.88161197 \\ \mathrm{H} & 23 & 1.09713176 & 20 & 111.18677102 & 17 & -59.99609807 \\ \mathrm{H} & 23 & 1.09712446 & 20 & 111.18948712 & 17 & 59.73979787\end{array}$

TS (6-endo) $(\mathrm{R}=\mathrm{Bu})$

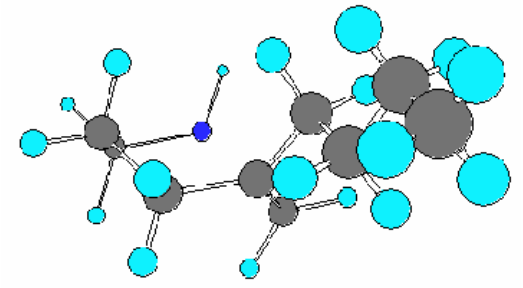

\#p UB3LYP/6-31 g* opt=(ts,z-matrix,noeigentest,nrscale) freq=noraman calculate for $\mathrm{TS}(6$-endo) $(\mathrm{R}=\mathrm{Bu}) \quad \mathrm{HF}=-408.4599802$

$\begin{array}{lrrrrrr}0 & 2 & & & & & \\ \mathrm{C} & & & & & & \\ \mathrm{C} & 1 & 1.54386611 & & & & \\ \mathrm{C} & 2 & 1.54958140 & 1 & 111.83925814 & & \\ \mathrm{C} & 3 & 1.51023412 & 2 & 109.28370386 & 1 & 55.95133525 \\ \mathrm{C} & 4 & 1.36905969 & 3 & 117.60056258 & 2 & -72.92105056 \\ \mathrm{H} & 1 & 1.10001116 & 2 & 109.25218554 & 3 & 62.89397754 \\ \mathrm{H} & 1 & 1.10490224 & 2 & 108.32151382 & 3 & 177.91568377 \\ \mathrm{H} & 2 & 1.09939764 & 1 & 109.79907522 & 3 & -122.47885042 \\ \mathrm{H} & 2 & 1.09990783 & 1 & 109.05154125 & 3 & 120.99780658 \\ \mathrm{H} & 3 & 1.09856522 & 2 & 108.57838502 & 1 & -64.77975807 \\ \mathrm{H} & 3 & 1.09736149 & 2 & 109.59004480 & 1 & 178.61729371 \\ \mathrm{H} & 5 & 1.08561821 & 4 & 121.06327154 & 3 & -26.42831879 \\ \mathrm{H} & 5 & 1.08693714 & 4 & 121.10477440 & 3 & 173.16135993 \\ \mathrm{~N} & 1 & 1.45864971 & 2 & 116.98679693 & 3 & -60.03175966 \\ \mathrm{H} & 14 & 1.03023493 & 1 & 106.60184281 & 2 & -48.75004304 \\ \mathrm{C} & 4 & 1.50894688 & 3 & 118.61232718 & 2 & 94.37149042\end{array}$




$\begin{array}{rrrrrrr}\mathrm{C} & 16 & 1.54432725 & 4 & 112.98199448 & 3 & 81.02838058 \\ \mathrm{H} & 16 & 1.10193574 & 4 & 110.13987681 & 3 & -40.07689837 \\ \mathrm{H} & 16 & 1.09819132 & 4 & 109.93064860 & 3 & -157.19922379 \\ \mathrm{C} & 17 & 1.53399493 & 16 & 113.15041039 & 4 & -177.40573667 \\ \mathrm{H} & 17 & 1.09944803 & 16 & 109.46540029 & 4 & -55.25455577 \\ \mathrm{H} & 17 & 1.09864019 & 16 & 108.87577607 & 4 & 60.35311887 \\ \mathrm{C} & 20 & 1.53215155 & 17 & 113.10066746 & 16 & -179.34943657 \\ \mathrm{H} & 20 & 1.09933771 & 17 & 109.24006388 & 16 & -57.17237995 \\ \mathrm{H} & 20 & 1.09938394 & 17 & 109.22103660 & 16 & 58.39739575 \\ \mathrm{H} & 23 & 1.09599089 & 20 & 111.40508936 & 17 & 179.98461409 \\ \mathrm{H} & 23 & 1.09700345 & 20 & 111.21046675 & 17 & -59.88141218 \\ \mathrm{H} & 23 & 1.09684623 & 20 & 111.15500065 & 17 & 59.87450474\end{array}$

\section{Radical A $(\mathbf{R}=\mathbf{i}-\mathbf{P r})$}

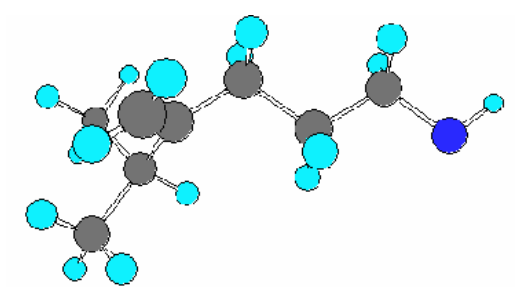

\#p UB3LYP/6-31 g* opt freq=noraman

calculate for Radical A $(\mathrm{R}=\mathrm{i}-\mathrm{Pr}) \quad \mathrm{HF}=-369.1554719$
$\begin{array}{ll}0 & 2\end{array}$
$\mathrm{C}$
C 11.33739536
$\begin{array}{lllll}\text { C } & 2 & 1.51798288 & 1 & 120.50701179\end{array}$
$\begin{array}{lllllll}\mathrm{C} & 3 & 1.54103700 & 2 & 113.10165287 & 1 & 100.58821815\end{array}$
$\begin{array}{lllllll}\mathrm{C} & 4 & 1.53004854 & 3 & 112.75137792 & 2 & 178.41142609\end{array}$
$\begin{array}{lllllll}\mathrm{H} & 1 & 1.08589683 & 2 & 122.74680419 & 3 & -177.82789395\end{array}$
$\begin{array}{llllllr}\mathrm{H} & 1 & 1.08774538 & 2 & 121.21729848 & 3 & 1.92313186\end{array}$
$\begin{array}{lllllll}\mathrm{H} & 3 & 1.10033175 & 2 & 109.97710492 & 1 & -137.62947537\end{array}$
$\begin{array}{lllllll}\mathrm{H} & 3 & 1.09780796 & 2 & 109.48659200 & 1 & -21.12493140\end{array}$
$\begin{array}{lllllll}\mathrm{H} & 4 & 1.09659533 & 3 & 109.55655143 & 2 & -60.08195914\end{array}$
$\begin{array}{lllllll}\mathrm{H} & 4 & 1.09719473 & 3 & 110.60813663 & 2 & 56.91844854\end{array}$
$\begin{array}{lllllll}\mathrm{H} & 5 & 1.10786682 & 4 & 110.14509039 & 3 & -57.27765761\end{array}$ 


$\begin{array}{rrrrrrr}\mathrm{H} & 5 & 1.10745745 & 4 & 110.21369801 & 3 & 57.56150372 \\ \mathrm{~N} & 5 & 1.44537227 & 4 & 111.75219806 & 3 & -179.64699008 \\ \mathrm{H} & 14 & 1.03219524 & 5 & 106.33041161 & 4 & -179.17365480 \\ \mathrm{C} & 2 & 1.52584515 & 1 & 123.39624314 & 3 & -179.65212249 \\ \mathrm{C} & 16 & 1.54447921 & 2 & 110.38469365 & 1 & 99.29900008 \\ \mathrm{H} & 17 & 1.09557675 & 16 & 111.98176499 & 2 & 54.00164160 \\ \mathrm{H} & 17 & 1.09653772 & 16 & 110.70226106 & 2 & 174.24681793 \\ \mathrm{H} & 17 & 1.09622108 & 16 & 110.36175015 & 2 & -66.03744005 \\ \mathrm{C} & 16 & 1.53481339 & 2 & 114.31700403 & 1 & -25.75722880 \\ \mathrm{H} & 21 & 1.09612566 & 16 & 110.29298294 & 2 & -174.52297920 \\ \mathrm{H} & 21 & 1.09514854 & 16 & 111.44613075 & 2 & -54.27210267 \\ \mathrm{H} & 21 & 1.09645825 & 16 & 111.51062162 & 2 & 66.45099866 \\ \mathrm{H} & 16 & 1.10036986 & 2 & 107.13456783 & 1 & -144.51433089\end{array}$

TS (5-exo) $(R=$ i-Pr)

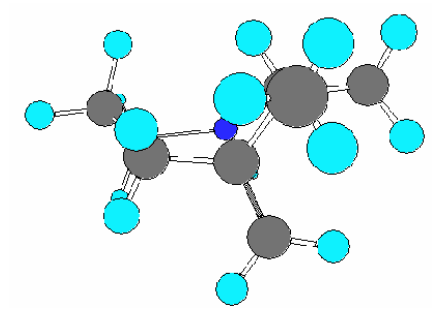

\#p UB3LYP/6-31 g* opt=(ts,z-matrix,noeigentest,nrscale) freq=noraman calculate for TS (5-exo) $(\mathrm{R}=\mathrm{i}-\mathrm{Pr}) \quad \mathrm{HF}=-369.1441477$
$\begin{array}{lll}0 & 2\end{array}$
$\mathrm{C}$
$\begin{array}{lll}\text { C } & 1 & 1.52898997\end{array}$
$\begin{array}{lllll}\mathrm{C} & 2 & 1.53832339 & 1 & 105.56406945\end{array}$
$\begin{array}{lllllll}\mathrm{C} & 3 & 1.53156902 & 2 & 111.38538985 & 1 & 43.91577099\end{array}$
$\begin{array}{lllllll}\mathrm{C} & 4 & 1.38364461 & 3 & 117.90679661 & 2 & -122.12015907\end{array}$
$\begin{array}{llllllr}\mathrm{H} & 1 & 1.10282880 & 2 & 110.14918546 & 3 & 70.33030041\end{array}$
$\begin{array}{lllllll}\mathrm{H} & 1 & 1.10273279 & 2 & 111.03289787 & 3 & -171.45725000\end{array}$
$\begin{array}{lllllll}\mathrm{H} & 2 & 1.09643942 & 1 & 112.59000022 & 3 & -122.08341993\end{array}$
$\begin{array}{lllllll}\mathrm{H} & 2 & 1.09662051 & 1 & 108.93574466 & 3 & 118.58803932\end{array}$
$\begin{array}{lllllll}\mathrm{H} & 3 & 1.09705501 & 2 & 109.05901587 & 1 & -76.52880724\end{array}$ 


$\begin{array}{rrrrrrr}\mathrm{H} & 3 & 1.09688141 & 2 & 110.58824659 & 1 & 166.54058553 \\ \mathrm{H} & 5 & 1.08657791 & 4 & 121.05778738 & 3 & 14.06381998 \\ \mathrm{H} & 5 & 1.08550704 & 4 & 122.13560892 & 3 & -171.12495020 \\ \mathrm{~N} & 1 & 1.45501258 & 2 & 105.19354162 & 3 & -53.67501877 \\ \mathrm{H} & 14 & 1.02668373 & 1 & 109.65312518 & 2 & 145.52203850 \\ \mathrm{C} & 4 & 1.53446205 & 3 & 116.04919094 & 2 & 84.11578311 \\ \mathrm{C} & 16 & 1.54337484 & 4 & 110.39752632 & 3 & 75.88630762 \\ \mathrm{H} & 17 & 1.09634673 & 16 & 110.82360672 & 4 & 65.83892756 \\ \mathrm{H} & 17 & 1.09654737 & 16 & 110.31848423 & 4 & -174.68978455 \\ \mathrm{H} & 17 & 1.09574593 & 16 & 112.35222714 & 4 & -54.67682732 \\ \mathrm{H} & 16 & 1.09727683 & 4 & 106.72820335 & 3 & -41.22279388 \\ \mathrm{C} & 16 & 1.53396354 & 4 & 114.00435845 & 3 & -159.39940811 \\ \mathrm{H} & 22 & 1.09280285 & 16 & 110.45604555 & 4 & 56.10148046 \\ \mathrm{H} & 22 & 1.09649878 & 16 & 110.04335083 & 4 & 175.82078074 \\ \mathrm{H} & 22 & 1.09694897 & 16 & 111.84751205 & 4 & -65.35510431\end{array}$

TS (6-endo) $(\mathrm{R}=\mathrm{i}$-Pr)

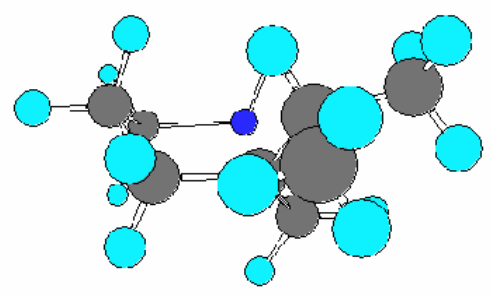

\#p UB3LYP/6-31 g* opt=(ts,z-matrix,noeigentest,nrscale) freq=noraman calculate for TS (6-endo) $(\mathrm{R}=\mathrm{i}-\mathrm{Pr}) \quad \mathrm{HF}=-369.1446904$
$\begin{array}{ll}0 & 2\end{array}$
$\mathrm{C}$
C $\quad 1 \quad 1.54275849$
$\begin{array}{lllll}\text { C } & 2 & 1.54984809 & 1 & 112.20231567\end{array}$
$\begin{array}{lllllll}\mathrm{C} & 3 & 1.51255711 & 2 & 109.67755189 & 1 & 55.36672340\end{array}$
$\begin{array}{lllllll}\mathrm{C} & 4 & 1.36954721 & 3 & 116.84361793 & 2 & -72.13054666\end{array}$
$\begin{array}{lllllll}\mathrm{H} & 1 & 1.09996714 & 2 & 109.34083066 & 3 & 63.38032684\end{array}$
$\begin{array}{lllllll}\mathrm{H} & 1 & 1.10481708 & 2 & 108.27126168 & 3 & 178.43451414\end{array}$
$\begin{array}{lllllll}\mathrm{H} & 2 & 1.09938541 & 1 & 109.78905733 & 3 & -122.59601899\end{array}$ 


$\begin{array}{rrrrrrr}\mathrm{H} & 2 & 1.10013457 & 1 & 108.94194566 & 3 & 121.01631396 \\ \mathrm{H} & 3 & 1.09880219 & 2 & 108.56155259 & 1 & -65.49189015 \\ \mathrm{H} & 3 & 1.09712954 & 2 & 109.31868491 & 1 & 178.13572895 \\ \mathrm{H} & 5 & 1.08550260 & 4 & 120.31895412 & 3 & -26.06828760 \\ \mathrm{H} & 5 & 1.08487509 & 4 & 122.08882602 & 3 & 173.76590518 \\ \mathrm{~N} & 1 & 1.45816667 & 2 & 116.89484341 & 3 & -59.52177864 \\ \mathrm{H} & 14 & 1.03036353 & 1 & 106.53344189 & 2 & -48.79561891 \\ \mathrm{C} & 4 & 1.51852064 & 3 & 117.52499071 & 2 & 96.62501481 \\ \mathrm{C} & 16 & 1.54621839 & 4 & 110.08003234 & 3 & 77.42699076 \\ \mathrm{H} & 17 & 1.09670882 & 16 & 110.69558174 & 4 & -173.86512219 \\ \mathrm{H} & 17 & 1.09542574 & 16 & 111.82437791 & 4 & -53.61819619 \\ \mathrm{H} & 17 & 1.09642126 & 16 & 110.44714897 & 4 & 66.38937503 \\ \mathrm{C} & 16 & 1.53445168 & 4 & 114.68614111 & 3 & -157.70929542 \\ \mathrm{H} & 21 & 1.09583268 & 16 & 110.27491656 & 4 & 176.48836991 \\ \mathrm{H} & 21 & 1.09665929 & 16 & 111.44149802 & 4 & -64.39364968 \\ \mathrm{H} & 21 & 1.09573597 & 16 & 111.85176041 & 4 & 56.38597642 \\ \mathrm{H} & 16 & 1.10301807 & 4 & 107.40453455 & 3 & -38.30441987\end{array}$

$\operatorname{Radical} A(\mathbf{R}=\mathbf{t}-\mathbf{B u})$

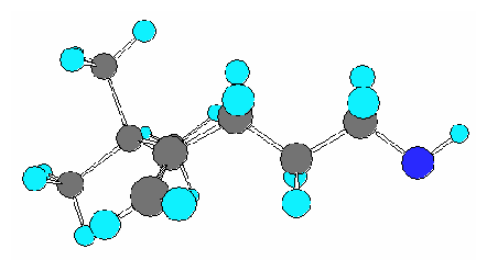

\#p UB3LYP/6-31 g* opt freq=noraman

calculate for Radical A $(\mathrm{R}=\mathrm{t}-\mathrm{Bu}) \quad \mathrm{HF}=-408.4650073$
$\begin{array}{ll}0 & 2\end{array}$
$\mathrm{C}$
$\begin{array}{lll}\text { C } & 1 & 1.33831283\end{array}$
$\begin{array}{lllll}\mathrm{C} & 2 & 1.52688540 & 1 & 118.55715754\end{array}$
$\begin{array}{lllllll}\text { C } & 3 & 1.54094539 & 2 & 113.59472024 & 1 & 77.24991609\end{array}$
$\begin{array}{lllllll}\text { C } & 4 & 1.52993729 & 3 & 112.72525157 & 2 & -179.71496305\end{array}$
$\begin{array}{lllllll}\mathrm{H} & 1 & 1.08418101 & 2 & 123.40348367 & 3 & -178.25031235\end{array}$
$\begin{array}{lllllll}\mathrm{H} & 1 & 1.08835413 & 2 & 120.83141112 & 3 & 1.43243928\end{array}$ 


$\begin{array}{rrrrrrr}\mathrm{H} & 3 & 1.09684930 & 2 & 110.79504381 & 1 & -159.67470585 \\ \mathrm{H} & 3 & 1.09836413 & 2 & 108.88016748 & 1 & -43.67368327 \\ \mathrm{H} & 4 & 1.09636351 & 3 & 109.55112184 & 2 & -58.32588277 \\ \mathrm{H} & 4 & 1.09613535 & 3 & 110.57732146 & 2 & 58.49312980 \\ \mathrm{H} & 5 & 1.10790081 & 4 & 110.23463312 & 3 & -57.91550565 \\ \mathrm{H} & 5 & 1.10773379 & 4 & 110.23501568 & 3 & 56.93148856 \\ \mathrm{~N} & 5 & 1.44560385 & 4 & 111.73825164 & 3 & 179.64781405 \\ \mathrm{H} & 14 & 1.03222583 & 5 & 106.32554722 & 4 & -179.57356158 \\ \mathrm{C} & 2 & 1.53682798 & 1 & 123.25785497 & 3 & -179.45672909 \\ \mathrm{C} & 16 & 1.54949285 & 2 & 108.94229636 & 1 & 112.26693971 \\ \mathrm{H} & 17 & 1.09541462 & 16 & 112.20076920 & 2 & 59.95456969 \\ \mathrm{H} & 17 & 1.09695860 & 16 & 110.28739937 & 2 & 179.67871501 \\ \mathrm{H} & 17 & 1.09585655 & 16 & 110.79556653 & 2 & -60.69470491 \\ \mathrm{C} & 16 & 1.53993175 & 2 & 112.25851539 & 1 & -7.31203627 \\ \mathrm{H} & 21 & 1.09614040 & 16 & 109.88910100 & 2 & -178.89784309 \\ \mathrm{H} & 21 & 1.09573133 & 16 & 111.54369526 & 2 & -59.41322561 \\ \mathrm{H} & 21 & 1.09554519 & 16 & 111.81004700 & 2 & 61.85630208 \\ \mathrm{C} & 16 & 1.54686256 & 2 & 110.54126440 & 1 & -127.97682689 \\ \mathrm{H} & 25 & 1.09521226 & 16 & 112.29566196 & 2 & -59.52047394 \\ \mathrm{H} & 25 & 1.09597836 & 16 & 111.12839254 & 2 & 61.56163367 \\ \mathrm{H} & 25 & 1.09675660 & 16 & 110.06103317 & 2 & -179.03570001\end{array}$

\section{$\operatorname{TS}(\mathbf{5}-\mathbf{e x o})(\mathbf{R}=\mathbf{t}-\mathbf{B u})$}

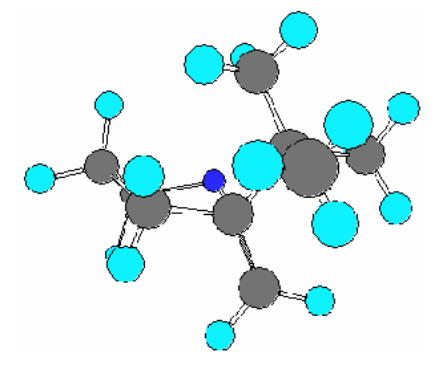

\#p UB3LYP/6-31 g* opt=(ts,z-matrix,noeigentest,nrscale) freq=noraman calculate for TS (5-exo) $(\mathrm{R}=\mathrm{t}-\mathrm{Bu}) \quad \mathrm{HF}=-408.4531059$

$\begin{array}{ll}0 & 2\end{array}$

$\mathrm{C}$ 


$\begin{array}{lrrrrrr}\mathrm{C} & 1 & 1.52931043 & & & & \\ \mathrm{C} & 2 & 1.54223858 & 1 & 106.52977212 & & \\ \mathrm{C} & 3 & 1.53572804 & 2 & 112.47801315 & 1 & 37.78065084 \\ \mathrm{C} & 4 & 1.38320672 & 3 & 116.32611583 & 2 & -112.76161885 \\ \mathrm{H} & 1 & 1.10243738 & 2 & 110.31784198 & 3 & 71.39079804 \\ \mathrm{H} & 1 & 1.10274289 & 2 & 110.74545846 & 3 & -170.60566463 \\ \mathrm{H} & 2 & 1.09670590 & 1 & 112.11047489 & 3 & -121.85955109 \\ \mathrm{H} & 2 & 1.09468143 & 1 & 108.91055797 & 3 & 119.25545747 \\ \mathrm{H} & 3 & 1.09633418 & 2 & 108.64526810 & 1 & -82.07112695 \\ \mathrm{H} & 3 & 1.09609754 & 2 & 110.55093576 & 1 & 161.65433245 \\ \mathrm{H} & 5 & 1.08651592 & 4 & 120.83366197 & 3 & 12.75732005 \\ \mathrm{H} & 5 & 1.08458848 & 4 & 122.63986393 & 3 & -172.21463984 \\ \mathrm{~N} & 1 & 1.45528351 & 2 & 105.65395983 & 3 & -52.66185719 \\ \mathrm{H} & 14 & 1.02678613 & 1 & 109.42010994 & 2 & 146.91510122 \\ \mathrm{C} & 4 & 1.55078621 & 3 & 118.54265764 & 2 & 93.69525570 \\ \mathrm{C} & 16 & 1.54149059 & 4 & 111.56863675 & 3 & -162.26047525 \\ \mathrm{H} & 17 & 1.09375604 & 16 & 110.42992906 & 4 & 58.85207336 \\ \mathrm{H} & 17 & 1.09654120 & 16 & 109.76804217 & 4 & 178.09962381 \\ \mathrm{H} & 17 & 1.09525700 & 16 & 112.52060789 & 4 & -62.89545588 \\ \mathrm{C} & 16 & 1.54179813 & 4 & 112.64164499 & 3 & -40.82992589 \\ \mathrm{H} & 21 & 1.09598245 & 16 & 112.22977474 & 4 & 59.10767860 \\ \mathrm{H} & 21 & 1.09667260 & 16 & 109.51623932 & 4 & 177.82770005 \\ \mathrm{H} & 21 & 1.09246061 & 16 & 110.78460076 & 4 & -62.53786493 \\ \mathrm{H} & 25 & 1.09665996 & 16 & 111.76429630 & 4 & -59.81545794\end{array}$

TS (6-endo) $(R=t-B u)$

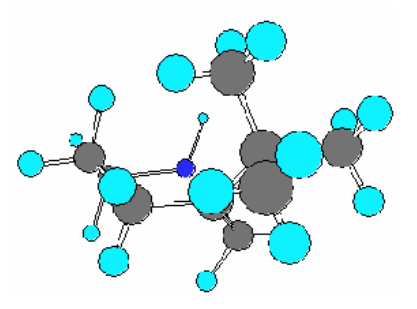




\begin{tabular}{|c|c|c|c|c|c|c|}
\hline \multicolumn{4}{|c|}{ calculate for $\mathrm{TS}$ (6-endo) $(\mathrm{R}=\mathrm{t}-\mathrm{Bu})$} & \multicolumn{3}{|c|}{$\mathrm{HF}=-408.4551599$} \\
\hline \multirow{2}{*}{\multicolumn{7}{|c|}{02}} \\
\hline & & & & & & \\
\hline $\mathrm{C}$ & 1 & 1.54262729 & & & & \\
\hline $\mathrm{C}$ & 2 & 1.55014553 & 1 & 112.76594583 & & \\
\hline $\mathrm{C}$ & 3 & 1.51701919 & 2 & 111.06209103 & 1 & 53.43697874 \\
\hline $\mathrm{C}$ & 4 & 1.37031594 & 3 & 115.55949425 & 2 & -70.04749256 \\
\hline $\mathrm{H}$ & 1 & 1.09997098 & 2 & 109.48461890 & 3 & 64.20525624 \\
\hline $\mathrm{H}$ & 1 & 1.10480196 & 2 & 108.19598945 & 3 & 179.29097390 \\
\hline $\mathrm{H}$ & 2 & 1.09958081 & 1 & 109.49507322 & 3 & -122.27303950 \\
\hline $\mathrm{H}$ & 2 & 1.09825912 & 1 & 108.80678360 & 3 & 121.56928968 \\
\hline $\mathrm{H}$ & 3 & 1.09912637 & 2 & 108.21644181 & 1 & -66.87923182 \\
\hline $\mathrm{H}$ & 3 & 1.09571313 & 2 & 109.10580763 & 1 & 177.62146610 \\
\hline $\mathrm{H}$ & 5 & 1.08558126 & 4 & 120.15830101 & 3 & -24.62451363 \\
\hline $\mathrm{H}$ & 5 & 1.08381910 & 4 & 122.50433827 & 3 & 175.19165968 \\
\hline $\mathrm{N}$ & 1 & 1.45790617 & 2 & 116.76659600 & 3 & -58.70896146 \\
\hline $\mathrm{H}$ & 14 & 1.03023639 & 1 & 106.64042994 & 2 & -48.19136775 \\
\hline $\mathrm{C}$ & 4 & 1.53162728 & 3 & 119.80557452 & 2 & 102.69328799 \\
\hline $\mathrm{C}$ & 16 & 1.55119358 & 4 & 108.08829278 & 3 & 69.16542115 \\
\hline $\mathrm{H}$ & 17 & 1.09712527 & 16 & 110.34215064 & 4 & -179.57998502 \\
\hline $\mathrm{H}$ & 17 & 1.09554626 & 16 & 111.90580111 & 4 & -59.81175415 \\
\hline $\mathrm{H}$ & 17 & 1.09569705 & 16 & 110.84058937 & 4 & 60.66482231 \\
\hline $\mathrm{C}$ & 16 & 1.54038009 & 4 & 112.19095496 & 3 & -171.74526671 \\
\hline $\mathrm{H}$ & 21 & 1.09601006 & 16 & 109.94153169 & 4 & -179.10027115 \\
\hline $\mathrm{H}$ & 21 & 1.09567015 & 16 & 111.72030053 & 4 & -59.78588361 \\
\hline $\mathrm{H}$ & 21 & 1.09601339 & 16 & 111.72950761 & 4 & 61.41299281 \\
\hline $\mathrm{C}$ & 16 & 1.54803862 & 4 & 111.60125077 & 3 & -50.17878795 \\
\hline $\mathrm{H}$ & 25 & 1.09632673 & 16 & 111.22467574 & 4 & -63.49707356 \\
\hline $\mathrm{H}$ & 25 & 1.09520070 & 16 & 112.19718170 & 4 & 57.59350687 \\
\hline $\mathrm{H}$ & 25 & 1.09682801 & 16 & 110.14926992 & 4 & 177.16829339 \\
\hline
\end{tabular}

\section{Radical A $(\mathbf{R}=\mathbf{P h})$}




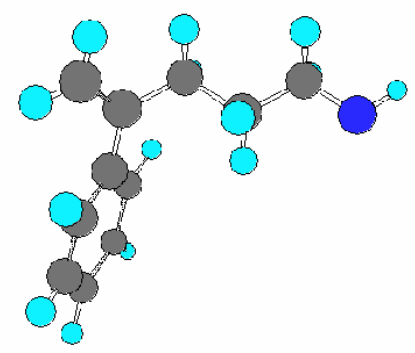

\#p UB3LYP/6-31g* opt freq=noraman

calculate for Radical A $(\mathrm{R}=\mathrm{Ph}) \quad \mathrm{HF}=-482.2681569$

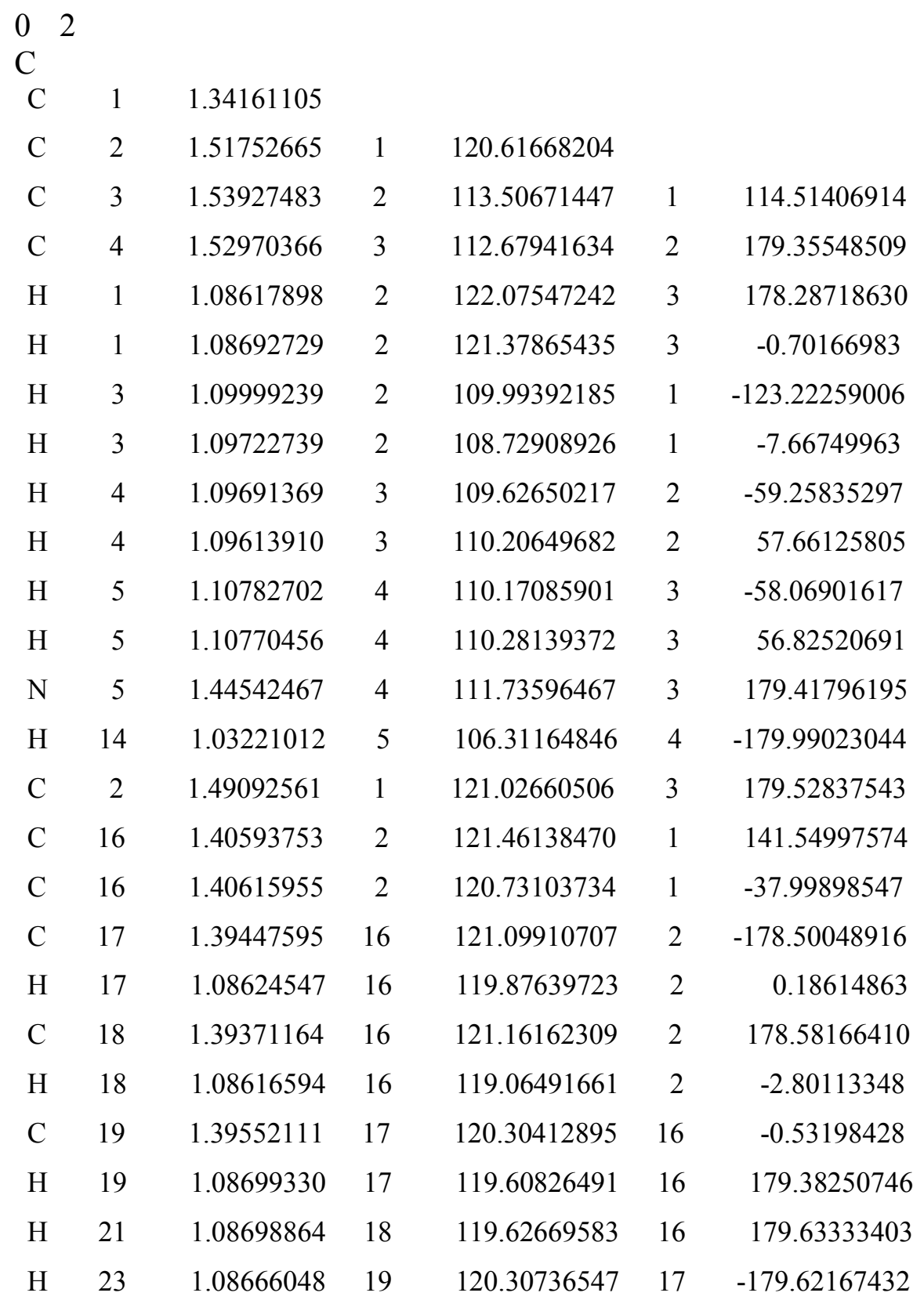


TS (5-exo) $(\mathbf{R}=\mathbf{P h})$

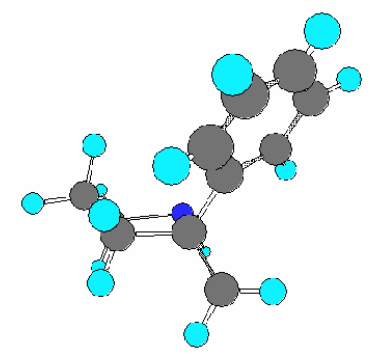

\#p UB3LYP/6-31 g* opt=(ts,z-matrix,noeigentest,nrscale) freq=noraman calculate for TS (5-exo) $(\mathrm{R}=\mathrm{Ph}) \quad \mathrm{HF}=-482.2510529$
$\begin{array}{ll}0 & 2\end{array}$
$\mathrm{C}$
$\begin{array}{lll}\mathrm{C} & 1 & 1.53031788\end{array}$
$\begin{array}{lllll}\text { C } & 2 & 1.53394934 & 1 & 104.98416550\end{array}$
$\begin{array}{lllllll}\mathrm{C} & 3 & 1.53055231 & 2 & 110.57080761 & 1 & 48.14565062\end{array}$
$\begin{array}{lllllll}\text { C } & 4 & 1.38943548 & 3 & 118.27411860 & 2 & -127.97775548\end{array}$
$\begin{array}{llllllr}\mathrm{H} & 1 & 1.10268496 & 2 & 110.07922765 & 3 & 71.80921437\end{array}$
$\begin{array}{lllllll}\mathrm{H} & 1 & 1.10229233 & 2 & 110.99077451 & 3 & -169.90178896\end{array}$
$\begin{array}{lllllll}\mathrm{H} & 2 & 1.09618530 & 1 & 112.53759681 & 3 & -122.08915860\end{array}$
$\begin{array}{lllllll}\mathrm{H} & 2 & 1.09639901 & 1 & 109.20596494 & 3 & 117.89573608\end{array}$
$\begin{array}{lllllll}\mathrm{H} & 3 & 1.09703980 & 2 & 109.34323342 & 1 & -71.50308248\end{array}$
$\begin{array}{lllllll}\mathrm{H} & 3 & 1.09537638 & 2 & 111.01070500 & 1 & 171.06097734\end{array}$
$\begin{array}{llllllr}\mathrm{H} & 5 & 1.08687611 & 4 & 121.11169366 & 3 & 17.08351909\end{array}$
$\begin{array}{lllllll}\mathrm{H} & 5 & 1.08565462 & 4 & 121.30745090 & 3 & -167.68086363\end{array}$
$\begin{array}{lllllll}\mathrm{N} & 1 & 1.45596998 & 2 & 105.34517106 & 3 & -52.36155495\end{array}$
$\begin{array}{lllllll}\mathrm{H} & 14 & 1.02701539 & 1 & 109.71444022 & 2 & 141.71680917\end{array}$
$\begin{array}{lllllll}\text { C } & 4 & 1.50877129 & 3 & 118.04572772 & 2 & 81.15137416\end{array}$
$\begin{array}{lllllll}\text { C } & 16 & 1.40296229 & 4 & 120.47201349 & 3 & 43.30111602\end{array}$
$\begin{array}{lllllll}\text { C } & 16 & 1.40244322 & 4 & 121.26456072 & 3 & -140.68876922\end{array}$
$\begin{array}{lllllll}\text { C } & 17 & 1.39621718 & 16 & 120.99985828 & 4 & 176.36647634\end{array}$
$\begin{array}{lllllll}\mathrm{H} & 17 & 1.08638976 & 16 & 120.10697482 & 4 & -2.45152751\end{array}$
$\begin{array}{lllllll}\text { C } & 18 & 1.39396051 & 16 & 120.92742823 & 4 & -176.04822244\end{array}$
$\begin{array}{lllllll}\mathrm{H} & 18 & 1.08394664 & 16 & 118.78385537 & 4 & 6.10791176\end{array}$ 


$\begin{array}{llllllr}\mathrm{C} & 19 & 1.39436234 & 17 & 120.23780888 & 16 & -0.27987866 \\ \mathrm{H} & 19 & 1.08709808 & 17 & 119.56220847 & 16 & -179.97343276 \\ \mathrm{H} & 21 & 1.08716129 & 18 & 119.56114059 & 16 & -179.65887898 \\ \mathrm{H} & 23 & 1.08686008 & 19 & 120.32672659 & 17 & 179.90961501\end{array}$

TS (6-endo) $(\mathrm{R}=\mathbf{P h})$

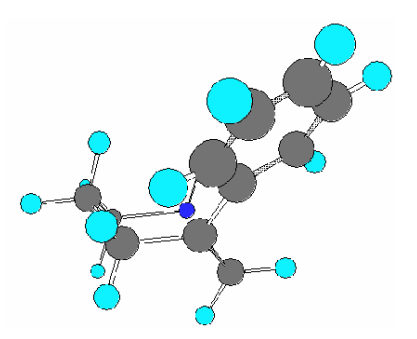

\#p UB3LYP/6-31g* opt=(ts,z-matrix,noeigentest,nrscale) freq=noraman calculate for TS (6-endo) $(\mathrm{R}=\mathrm{Ph}) \quad \mathrm{HF}=-482.2596358$

$\begin{array}{rrrrrrr}0 & 2 & & & & & \\ \mathrm{C} & & & & & \\ \mathrm{C} & 1 & 1.54350333 & & & & \\ \mathrm{C} & 2 & 1.54900512 & 1 & 112.33643758 & & \\ \mathrm{C} & 3 & 1.51524566 & 2 & 109.46961053 & 1 & 56.85806136 \\ \mathrm{C} & 4 & 1.36807658 & 3 & 116.19911703 & 2 & -76.25568015 \\ \mathrm{H} & 1 & 1.09979404 & 2 & 109.50061054 & 3 & 63.18490430 \\ \mathrm{H} & 1 & 1.10549387 & 2 & 108.11079050 & 3 & 178.16156480 \\ \mathrm{H} & 2 & 1.09918592 & 1 & 109.50726291 & 3 & -122.26038418 \\ \mathrm{H} & 2 & 1.09917368 & 1 & 109.20448939 & 3 & 121.10101551 \\ \mathrm{H} & 3 & 1.09768182 & 2 & 108.83691720 & 1 & -63.27653006 \\ \mathrm{H} & 3 & 1.09430584 & 2 & 108.99043074 & 1 & -179.50727681 \\ \mathrm{H} & 5 & 1.08410493 & 4 & 120.53576392 & 3 & -21.00053442 \\ \mathrm{H} & 5 & 1.08470764 & 4 & 122.33867895 & 3 & 175.77615546 \\ \mathrm{~N} & 1 & 1.45621521 & 2 & 116.94297804 & 3 & -60.14583207 \\ \mathrm{H} & 14 & 1.03034159 & 1 & 106.41995388 & 2 & -47.42661608 \\ \mathrm{C} & 4 & 1.47759340 & 3 & 120.40199791 & 2 & 92.17858941 \\ \mathrm{C} & 16 & 1.40937781 & 4 & 121.22803290 & 3 & 34.30330962 \\ \mathrm{C} & 16 & 1.41170846 & 4 & 121.53395647 & 3 & -146.55641597 \\ \mathrm{C} & 17 & 1.39389660 & 16 & 121.36529153 & 4 & 178.54582187\end{array}$




$\begin{array}{llllllr}\mathrm{H} & 17 & 1.08557260 & 16 & 119.81421145 & 4 & 0.29077132 \\ \mathrm{C} & 18 & 1.39160039 & 16 & 121.38245926 & 4 & -178.73455041 \\ \mathrm{H} & 18 & 1.08601115 & 16 & 119.55188020 & 4 & 2.74820650 \\ \mathrm{C} & 19 & 1.39522781 & 17 & 120.44331985 & 16 & 0.47600048 \\ \mathrm{H} & 19 & 1.08704312 & 17 & 119.46990759 & 16 & -179.35579992 \\ \mathrm{H} & 21 & 1.08704667 & 18 & 119.49460679 & 16 & -179.46058751 \\ \mathrm{H} & 23 & 1.08650540 & 19 & 120.43880950 & 17 & 179.66948961\end{array}$

$\operatorname{Radical}$ A $(\mathbf{R}=\mathbf{C l})$

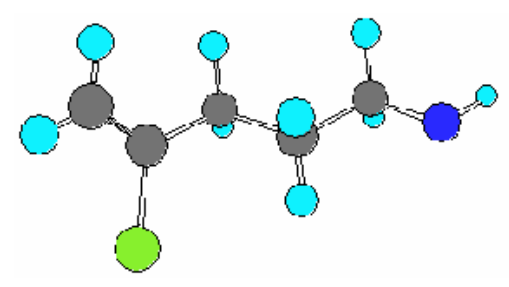

\#p UB3LYP/6-31 g* opt freq=noraman

calculate for Radical A $(\mathrm{R}=\mathrm{Cl}) \quad \mathrm{HF}=-710.8111238$

$\begin{array}{lrrrrrr}0 & 2 & & & & & \\ \mathrm{C} & & & & & & \\ \mathrm{C} & 1 & 1.33100792 & & & & \\ \mathrm{C} & 2 & 1.50393978 & 1 & 126.38748211 & & \\ \mathrm{C} & 3 & 1.53872173 & 2 & 114.11659382 & 1 & 111.70780516 \\ \mathrm{C} & 4 & 1.53025828 & 3 & 112.25063065 & 2 & 179.15722995 \\ \mathrm{H} & 1 & 1.08396939 & 2 & 122.55916156 & 3 & -179.57446183 \\ \mathrm{H} & 1 & 1.08637569 & 2 & 119.64879153 & 3 & 0.39503227 \\ \mathrm{H} & 3 & 1.09900056 & 2 & 108.92159277 & 1 & -125.66030391 \\ \mathrm{H} & 3 & 1.09793738 & 2 & 107.47633082 & 1 & -10.24177102 \\ \mathrm{H} & 4 & 1.09646061 & 3 & 109.74851709 & 2 & -59.46316181 \\ \mathrm{H} & 4 & 1.09546350 & 3 & 110.10973277 & 2 & 57.78548311 \\ \mathrm{H} & 5 & 1.10741904 & 4 & 110.14398455 & 3 & -58.30039066 \\ \mathrm{H} & 5 & 1.10760684 & 4 & 110.36407425 & 3 & 56.76622084 \\ \mathrm{~N} & 5 & 1.44489371 & 4 & 111.45069947 & 3 & 179.26592157 \\ \mathrm{H} & 4 & 1.03199512 & 5 & 106.34587990 & 4 & 179.88733121 \\ \mathrm{Cl} & 2 & 1.77467332 & 1 & 119.24744848 & 3 & 179.78697953\end{array}$


TS (5-exo) $(\mathbf{R}=\mathbf{C l})$

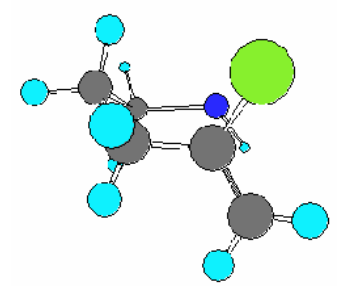

\#p UB3LYP/6-31 g* opt=(ts,z-matrix,noeigentest,nrscale) freq=noraman calculate for TS (5-exo) $(\mathrm{R}=\mathrm{Cl}) \quad \mathrm{HF}=-710.7943285$

$\begin{array}{lrrrrrr}0 & 2 & & & & & \\ \mathrm{C} & & & & & & \\ \mathrm{C} & 1 & 1.53133816 & & & & \\ \mathrm{C} & 2 & 1.53412361 & 1 & 105.35622354 & & \\ \mathrm{C} & 3 & 1.52094097 & 2 & 111.32997946 & 1 & 45.88770647 \\ \mathrm{C} & 4 & 1.37390534 & 3 & 122.32659003 & 2 & -128.04536438 \\ \mathrm{H} & 1 & 1.10307246 & 2 & 110.36036486 & 3 & 71.16466945 \\ \mathrm{H} & 1 & 1.10236704 & 2 & 110.91352131 & 3 & -170.48615334 \\ \mathrm{H} & 2 & 1.09569232 & 1 & 112.58097015 & 3 & -121.81233670 \\ \mathrm{H} & 2 & 1.09560850 & 1 & 109.20135309 & 3 & 118.05709653 \\ \mathrm{H} & 3 & 1.09774416 & 2 & 109.41143870 & 1 & -72.61252956 \\ \mathrm{H} & 3 & 1.09521972 & 2 & 111.94495083 & 1 & 168.58096192 \\ \mathrm{H} & 5 & 1.08595418 & 4 & 119.58635912 & 3 & 18.65938112 \\ \mathrm{H} & 5 & 1.08362223 & 4 & 121.92838274 & 3 & -167.08823164 \\ \mathrm{~N} & 1 & 1.45344343 & 2 & 105.11180513 & 3 & -53.30595352 \\ \mathrm{H} & 14 & 1.02763886 & 1 & 110.24908382 & 2 & 145.10843278 \\ \mathrm{Cl} & 4 & 1.78437233 & 3 & 114.38091602 & 2 & 81.49216712\end{array}$

TS (6-endo) $(\mathrm{R}=\mathrm{Cl})$

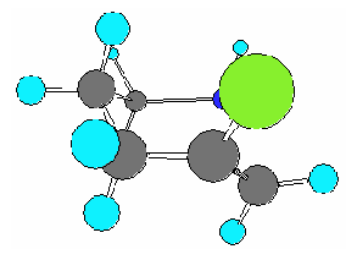




\begin{tabular}{|c|c|c|c|c|c|c|}
\hline \multicolumn{4}{|c|}{ calculate for TS (6-endo) $(\mathrm{R}=\mathrm{Cl})$} & \multicolumn{3}{|c|}{$\mathrm{HF}=-710.8001322$} \\
\hline \multirow{2}{*}{\multicolumn{7}{|c|}{2}} \\
\hline & & & & & & \\
\hline$C$ & 1 & 1.54406180 & & & & \\
\hline $\mathrm{C}$ & 2 & 1.54885897 & 1 & 111.72268582 & & \\
\hline $\mathrm{C}$ & 3 & 1.50152796 & 2 & 109.49675740 & 1 & 54.88205243 \\
\hline $\mathrm{C}$ & 4 & 1.36055294 & 3 & 121.50933903 & 2 & -71.69339806 \\
\hline $\mathrm{H}$ & 1 & 1.09981095 & 2 & 109.43074101 & 3 & 62.74262564 \\
\hline $\mathrm{H}$ & 1 & 1.10464458 & 2 & 108.25010463 & 3 & 177.82347252 \\
\hline $\mathrm{H}$ & 2 & 1.09859585 & 1 & 109.81443148 & 3 & -121.97712437 \\
\hline $\mathrm{H}$ & 2 & 1.09849684 & 1 & 109.47504355 & 3 & 120.95509713 \\
\hline $\mathrm{H}$ & 3 & 1.09843509 & 2 & 109.01534876 & 1 & -63.75327362 \\
\hline $\mathrm{H}$ & 3 & 1.09441977 & 2 & 110.63171124 & 1 & 178.19733186 \\
\hline $\mathrm{H}$ & 5 & 1.08476860 & 4 & 118.87460543 & 3 & -28.83294476 \\
\hline $\mathrm{H}$ & 5 & 1.08377317 & 4 & 122.07261812 & 3 & 170.23959655 \\
\hline $\mathrm{N}$ & 1 & 1.45830175 & 2 & 117.06997765 & 3 & -60.46437023 \\
\hline $\mathrm{H}$ & 14 & 1.03012298 & 1 & 106.75300627 & 2 & -48.10528156 \\
\hline $\mathrm{Cl}$ & 4 & 1.76722945 & 3 & 116.09598419 & 2 & 88.90417971 \\
\hline
\end{tabular}

\section{Radical A $(\mathbf{R}=\mathbf{B r})$}

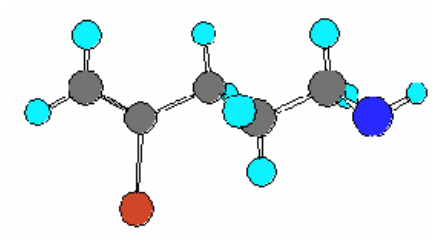

\#p UB3LYP/6-31g* opt freq=noraman

calculate for Radical A $(\mathrm{R}=\mathrm{Br}) \quad \mathrm{HF}=-2822.320366$
$\begin{array}{ll}0 & 2\end{array}$
C
$\begin{array}{lll}\text { C } & 1 & 1.32988949\end{array}$
$\begin{array}{lllll}\mathrm{C} & 2 & 1.50249990 & 1 & 126.91624395\end{array}$
$\begin{array}{lllllll}\mathrm{C} & 3 & 1.53863574 & 2 & 113.96671845 & 1 & 113.38461956\end{array}$
$\begin{array}{lllllll}\mathrm{C} & 4 & 1.52971366 & 3 & 112.41823529 & 2 & 178.44567170\end{array}$ 


$\begin{array}{lrrrrrr}\mathrm{H} & 1 & 1.08387430 & 2 & 122.78590831 & 3 & -179.59578720 \\ \mathrm{H} & 1 & 1.08750444 & 2 & 119.65990816 & 3 & 0.53481516 \\ \mathrm{H} & 3 & 1.09895877 & 2 & 108.98581859 & 1 & -124.16862749 \\ \mathrm{H} & 3 & 1.09863422 & 2 & 107.58337424 & 1 & -8.54951401 \\ \mathrm{H} & 4 & 1.09652649 & 3 & 109.71924952 & 2 & -60.04825061 \\ \mathrm{H} & 4 & 1.09526474 & 3 & 109.73063770 & 2 & 56.94475671 \\ \mathrm{H} & 5 & 1.10740026 & 4 & 110.16310658 & 3 & -58.48756348 \\ \mathrm{H} & 5 & 1.10765228 & 4 & 110.39443682 & 3 & 56.61260366 \\ \mathrm{~N} & 5 & 1.44497546 & 4 & 111.43207590 & 3 & 178.98607136 \\ \mathrm{H} & 14 & 1.03201258 & 5 & 106.33858819 & 4 & 179.45049173 \\ \mathrm{Br} & 2 & 1.93331940 & 1 & 118.90761292 & 3 & 179.68182124\end{array}$

$\operatorname{TS}(5$-exo) $(\mathbf{R}=\mathbf{B r})$

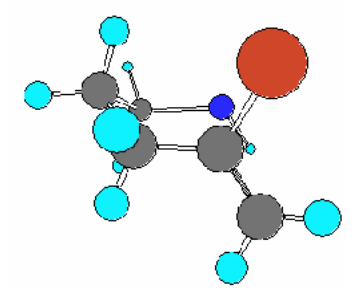

\#p UB3LYP/6-31 g* opt=(ts,z-matrix,noeigentest,nrscale) freq=noraman calculate for TS (5-exo) $(\mathrm{R}=\mathrm{Br}) \quad \mathrm{HF}=-2822.30437$

$\begin{array}{lrrrrrr}0 & 2 & & & & & \\ \mathrm{C} & & & & & & \\ \mathrm{C} & 1 & 1.53188873 & & & & \\ \mathrm{C} & 2 & 1.53355470 & 1 & 105.35584873 & & \\ \mathrm{C} & 3 & 1.51782935 & 2 & 110.90087477 & 1 & 46.95598544 \\ \mathrm{C} & 4 & 1.37213977 & 3 & 122.98940469 & 2 & -130.77671204 \\ \mathrm{H} & 1 & 1.10290638 & 2 & 110.29203545 & 3 & 71.38165512 \\ \mathrm{H} & 1 & 1.10230476 & 2 & 110.81521222 & 3 & -170.32553356 \\ \mathrm{H} & 2 & 1.09554166 & 1 & 112.54989757 & 3 & -121.97417102 \\ \mathrm{H} & 2 & 1.09580921 & 1 & 109.23742399 & 3 & 117.74603525 \\ \mathrm{H} & 3 & 1.09866046 & 2 & 109.53683792 & 1 & -71.68914885 \\ \mathrm{H} & 3 & 1.09516402 & 2 & 111.82813508 & 1 & 169.37459791 \\ \mathrm{H} & 5 & 1.08704417 & 4 & 119.59781025 & 3 & 18.50445195\end{array}$




$\begin{array}{rrrrrrr}\mathrm{H} & 5 & 1.08347671 & 4 & 122.13171724 & 3 & -167.05524304 \\ \mathrm{~N} & 1 & 1.45388299 & 2 & 105.33337952 & 3 & -53.18956528 \\ \mathrm{H} & 14 & 1.02769727 & 1 & 110.19422703 & 2 & 143.69060081 \\ \mathrm{Br} & 4 & 1.94560185 & 3 & 113.81500184 & 2 & 79.14542834\end{array}$

TS (6-endo) $(\mathrm{R}=\mathrm{Br})$

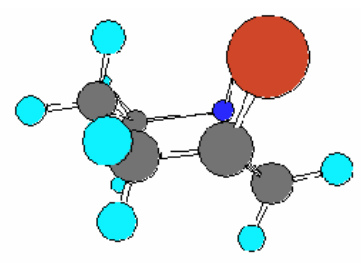

\#p UB3LYP/6-31 g* opt=(ts,z-matrix,noeigentest,nrscale) freq=noraman calculate for TS (6-endo) $(\mathrm{R}=\mathrm{Br}) \quad \mathrm{HF}=-2822.3097294$

$\begin{array}{lrrrrrr}0 & 2 & & & & & \\ \mathrm{C} & & & & & & \\ \mathrm{C} & 1 & 1.54464587 & & & & \\ \mathrm{C} & 2 & 1.54831991 & 1 & 111.61114815 & & \\ \mathrm{C} & 3 & 1.49990166 & 2 & 109.18458224 & 1 & 54.94841436 \\ \mathrm{C} & 4 & 1.35934304 & 3 & 121.90429473 & 2 & -73.07314850 \\ \mathrm{H} & 1 & 1.09974859 & 2 & 109.44503047 & 3 & 63.37789680 \\ \mathrm{H} & 1 & 1.10478348 & 2 & 108.21855054 & 3 & 178.41281106 \\ \mathrm{H} & 2 & 1.09846459 & 1 & 109.84402538 & 3 & -122.12366600 \\ \mathrm{H} & 2 & 1.09826137 & 1 & 109.56929410 & 3 & 120.60684777 \\ \mathrm{H} & 3 & 1.09888359 & 2 & 109.17086477 & 1 & -63.64654988 \\ \mathrm{H} & 3 & 1.09438918 & 2 & 110.53102940 & 1 & 178.14898622 \\ \mathrm{H} & 5 & 1.08588227 & 4 & 118.94444721 & 3 & -29.11729736 \\ \mathrm{H} & 5 & 1.08350598 & 4 & 122.27757612 & 3 & 169.40388591 \\ \mathrm{~N} & 1 & 1.45806597 & 2 & 117.18850718 & 3 & -59.92685528 \\ \mathrm{H} & 14 & 1.03009045 & 1 & 106.80633715 & 2 & -47.43021017 \\ \mathrm{Br} & 4 & 1.92537491 & 3 & 115.50062875 & 2 & 84.49008401\end{array}$

\section{$N$-butylpent-4-enylaminyl Radical}

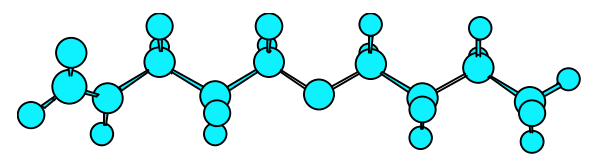




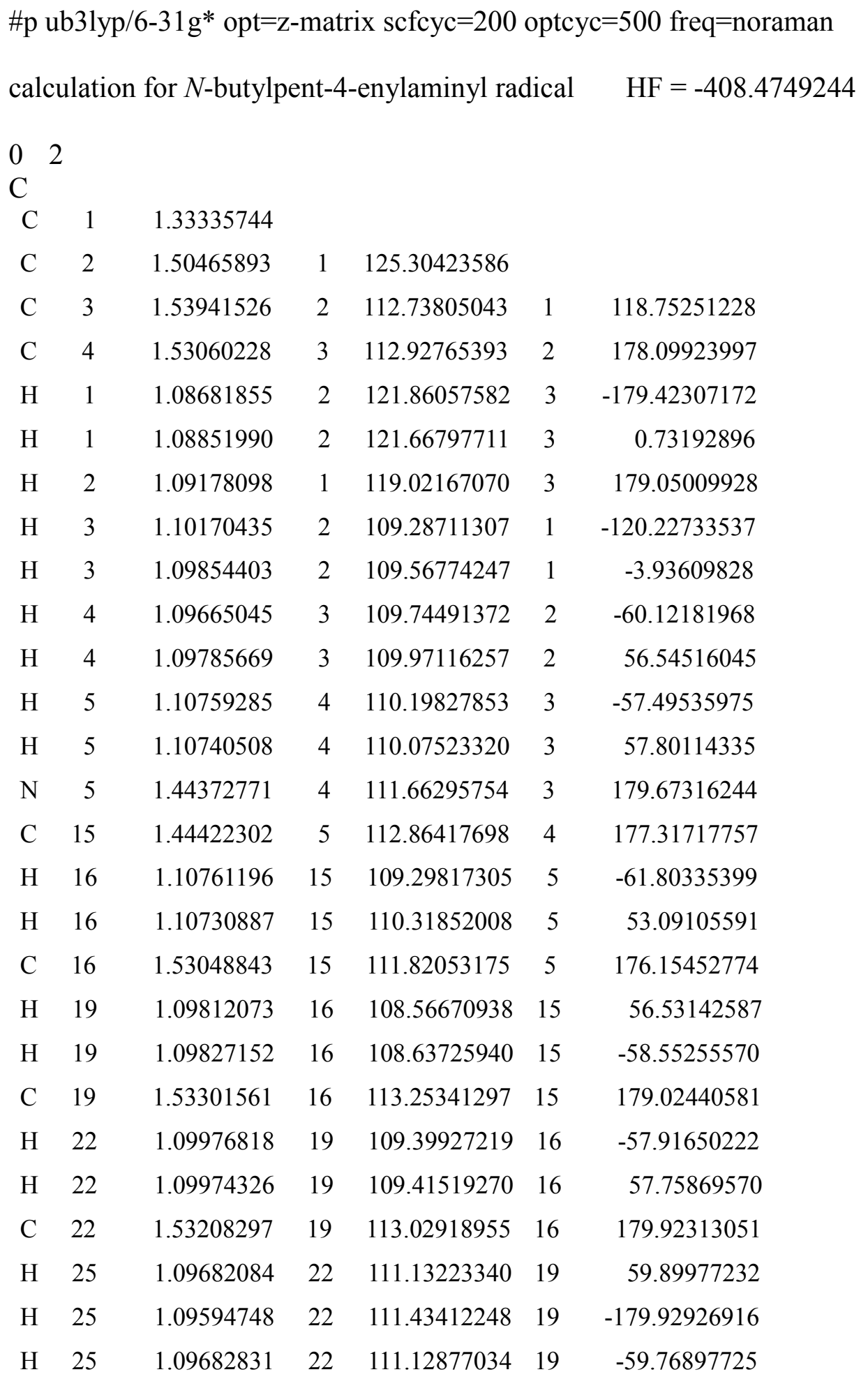

\section{TS (5-exo) ( $N$-butylpent-4-enylaminyl radical)}




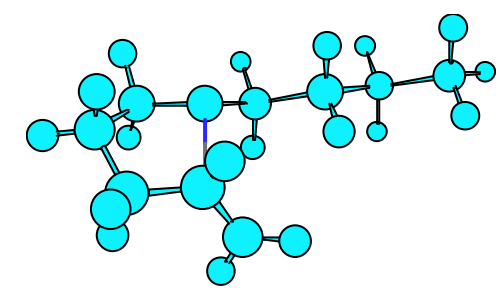

$\# p \quad$ ub3lyp/6-31g* opt=(ts,z-matrix,noeigentest,nrscale) $\quad$ scfcyc $=200 \quad$ optcyc $=500$ freq=noraman $\operatorname{iop}(1 / 8=5)$

calculation for TS (5-exo) $\quad \mathrm{HF}=-408.4454979$

$\begin{array}{crrrrrr}0 & 2 & & & & & \\ \mathrm{C} & & & & & & \\ \mathrm{N} & 1 & 2.00862443 & & & & \\ \mathrm{C} & 2 & 1.45171248 & 1 & 99.43109832 & & \\ \mathrm{C} & 3 & 1.52585502 & 2 & 105.67399918 & 1 & 38.06187546 \\ \mathrm{C} & 1 & 1.52309161 & 2 & 95.72220509 & 3 & -13.94435931 \\ \mathrm{C} & 1 & 1.38903069 & 5 & 119.63060588 & 4 & -133.43123581 \\ \mathrm{H} & 3 & 1.10714911 & 2 & 113.64405383 & 1 & -83.32807677 \\ \mathrm{H} & 3 & 1.10206607 & 2 & 108.61471560 & 1 & 158.03522224 \\ \mathrm{H} & 4 & 1.09586973 & 3 & 112.65618936 & 2 & -174.35675352 \\ \mathrm{H} & 4 & 1.09656467 & 3 & 108.99123276 & 2 & 65.89814231 \\ \mathrm{H} & 5 & 1.09792173 & 1 & 108.85595275 & 6 & -13.32562239 \\ \mathrm{H} & 5 & 1.09741626 & 1 & 109.79629180 & 6 & 103.20655527 \\ \mathrm{H} & 1 & 1.08652356 & 6 & 116.80150330 & 5 & 147.51147370 \\ \mathrm{H} & 6 & 1.08736291 & 1 & 121.14630390 & 5 & 21.96969350 \\ \mathrm{H} & 6 & 1.08587554 & 1 & 121.79283295 & 5 & -164.70019384 \\ \mathrm{C} & 2 & 1.45569149 & 1 & 115.76471401 & 6 & -10.93623992 \\ \mathrm{H} & 16 & 1.10388960 & 2 & 106.99924797 & 1 & 165.63175650 \\ \mathrm{H} & 16 & 1.10380868 & 2 & 112.62416276 & 1 & 50.27219834 \\ \mathrm{C} & 16 & 1.53050039 & 2 & 112.22728865 & 1 & -75.15831944 \\ \mathrm{H} & 19 & 1.09809057 & 16 & 108.43944930 & 2 & -54.68089235 \\ \mathrm{H} & 19 & 1.09778253 & 16 & 109.31055036 & 2 & 60.81773362 \\ \mathrm{H} & 19 & 1.53357166 & 16 & 113.13645279 & 2 & -176.95986573 \\ & 22 & 1.09958730 & 19 & 109.39169310 & 16 & -57.94349819 \\ & 22 & 1.53218130 & 19 & 113.10236939 & 16 & 179.88861425\end{array}$




$\begin{array}{llllllr}\mathrm{H} & 25 & 1.09703883 & 22 & 111.15808211 & 19 & 59.87271541 \\ \mathrm{H} & 25 & 1.09608837 & 22 & 111.46974981 & 19 & -179.97478782 \\ \mathrm{H} & 25 & 1.09693740 & 22 & 111.16061013 & 19 & -59.79673378\end{array}$

${ }^{1}$ H NMR spectra of new compounds.
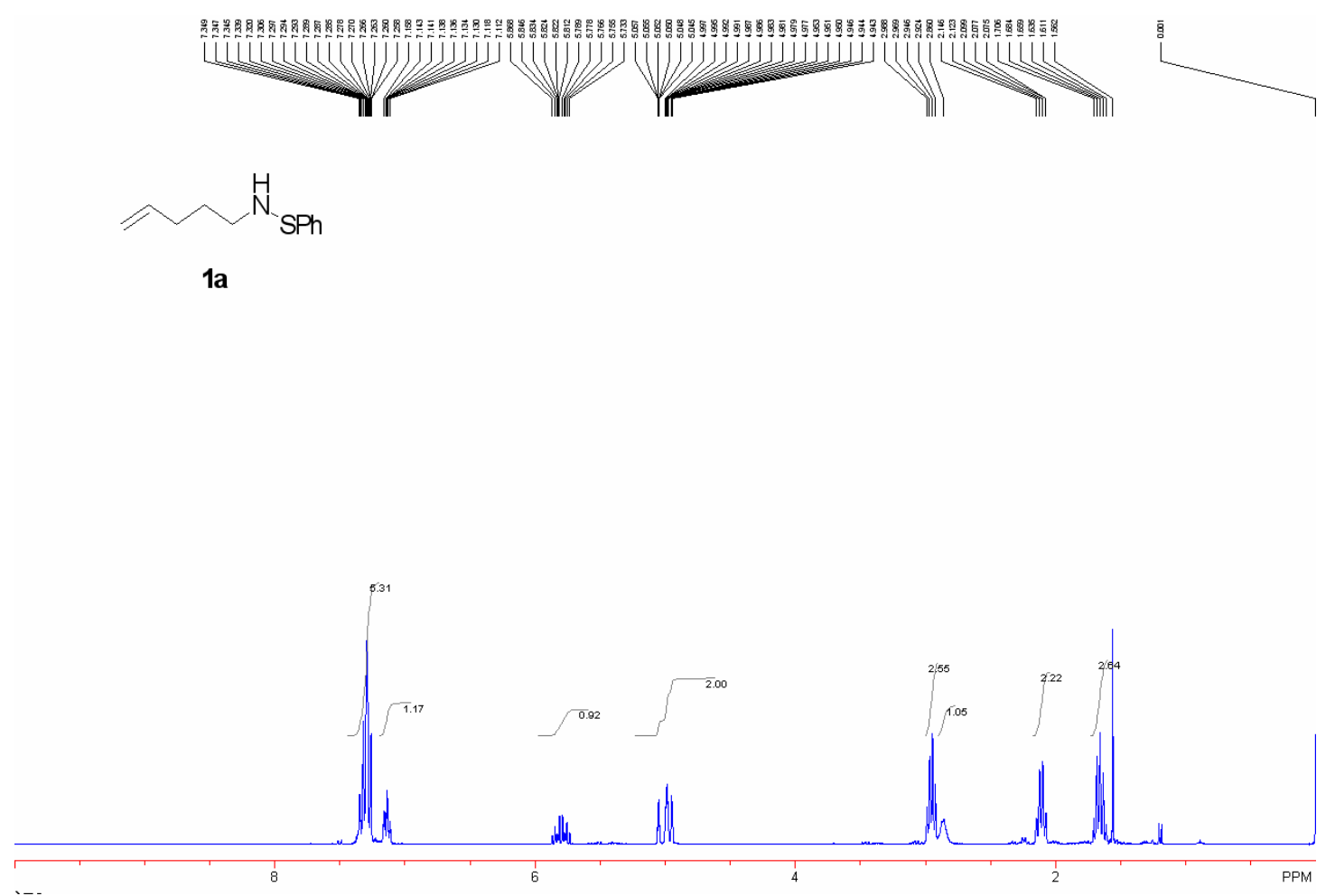

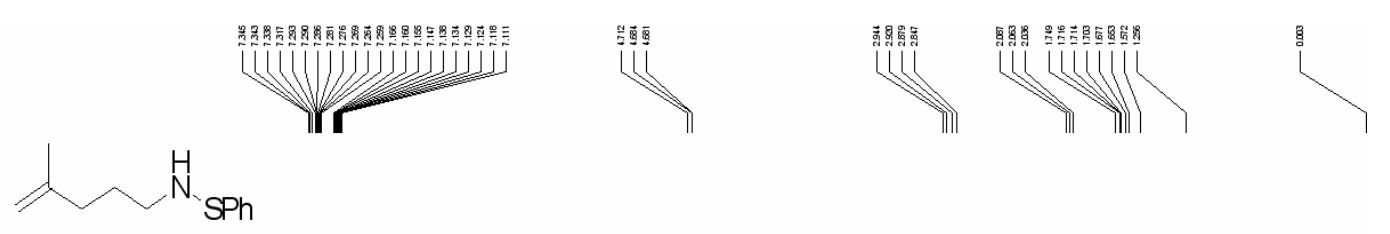

$1 b$
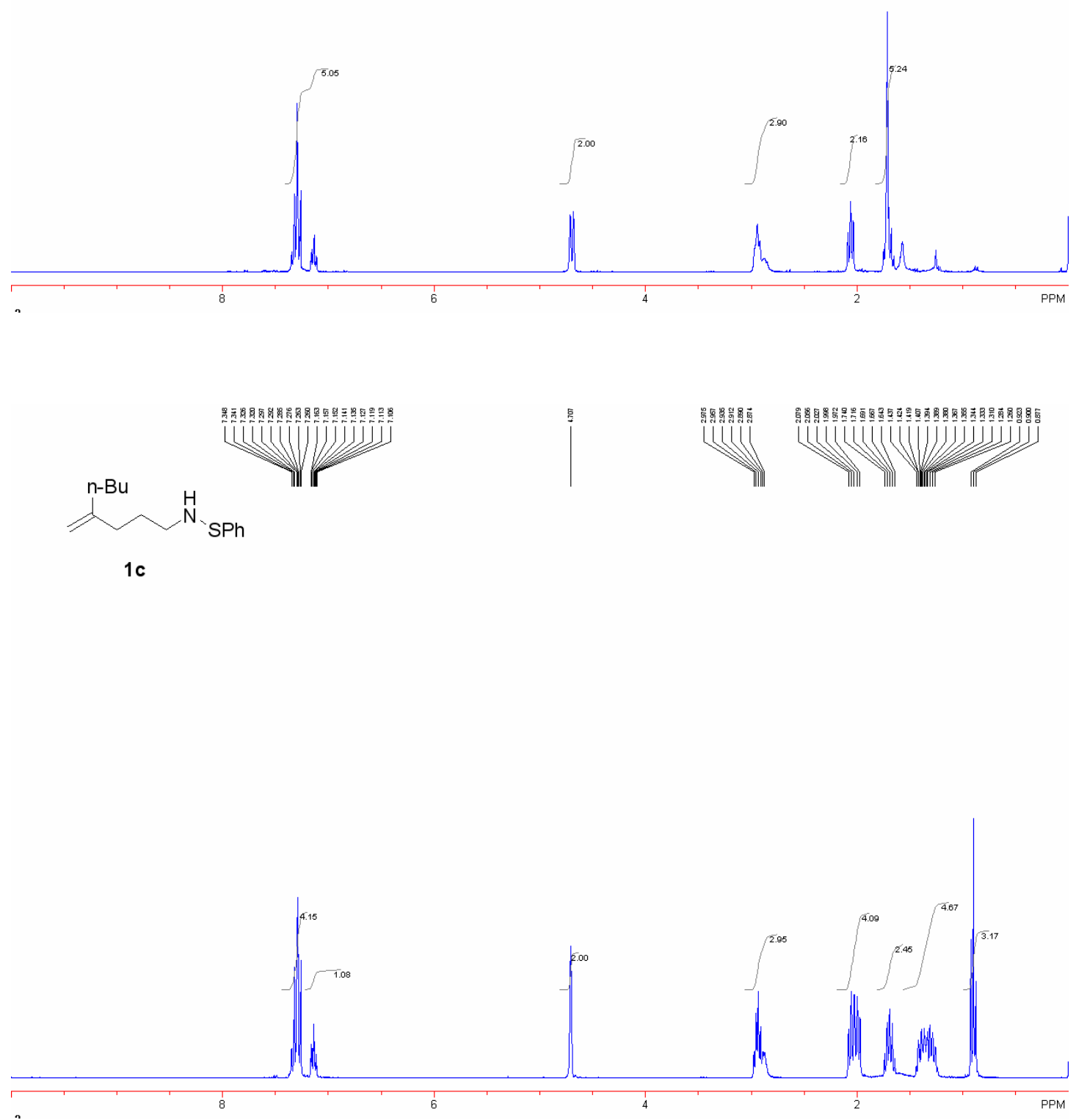


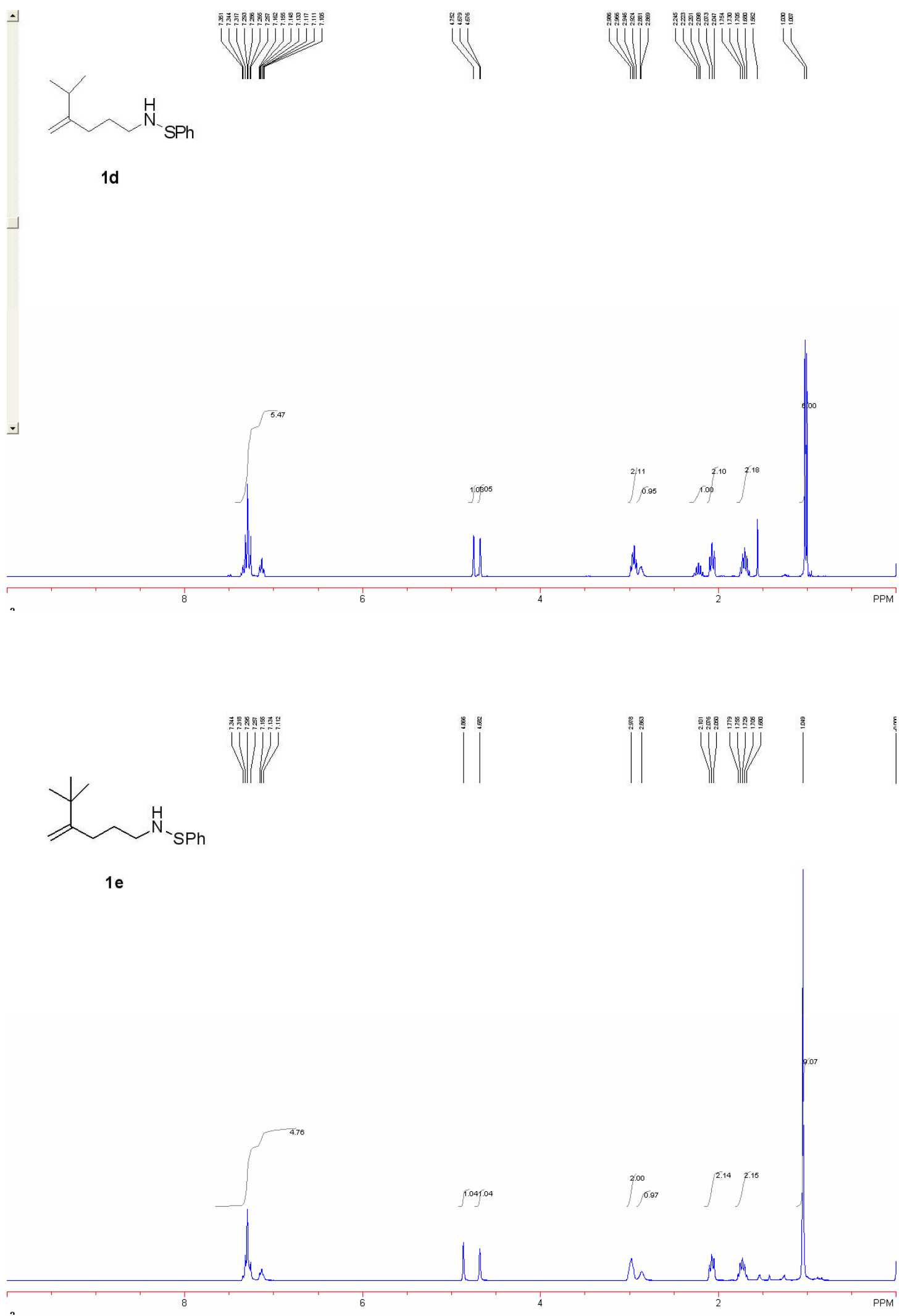



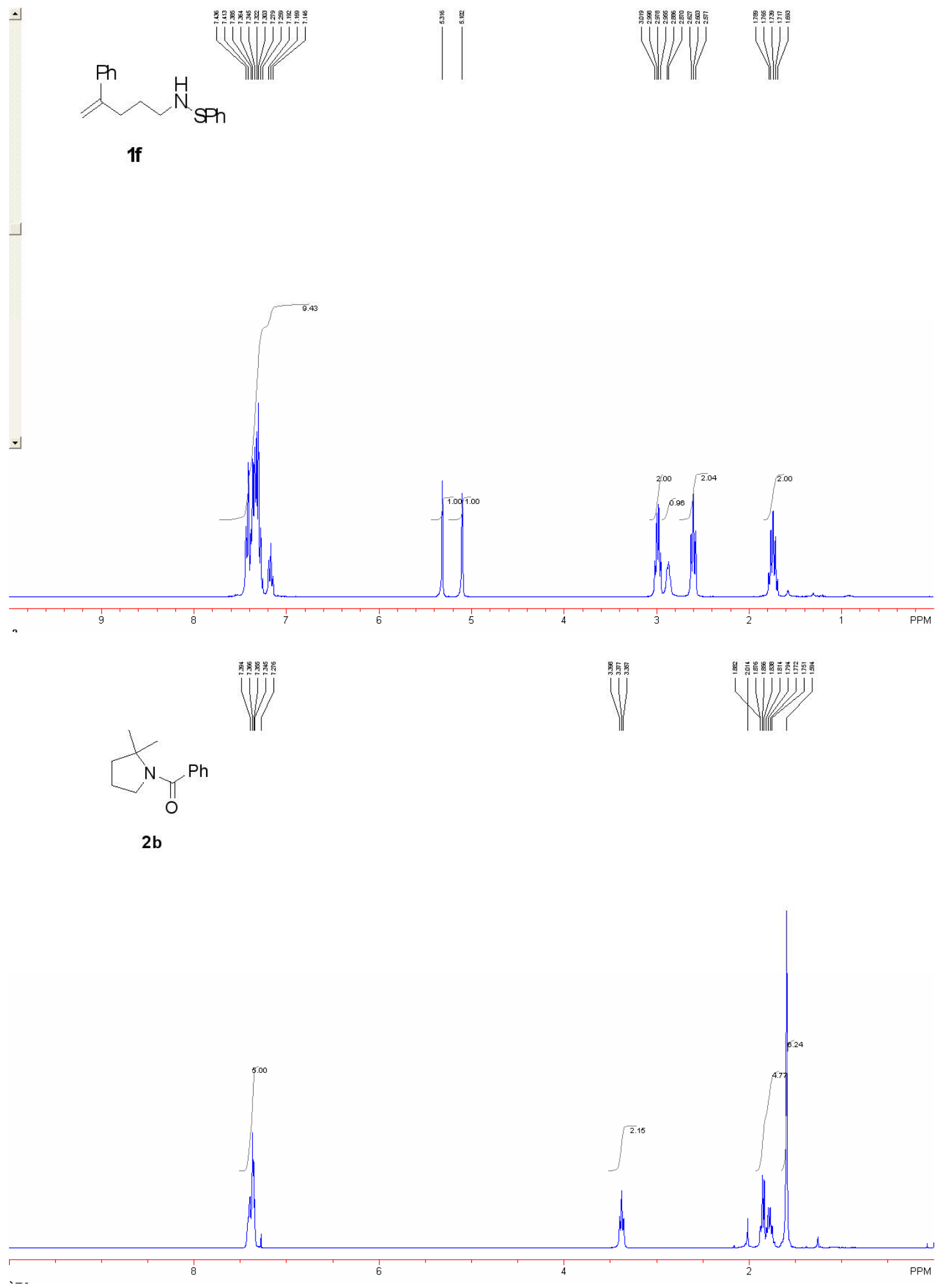

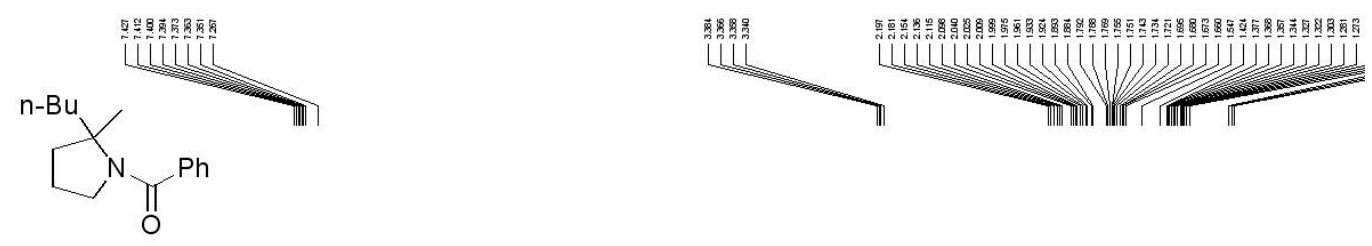

2c
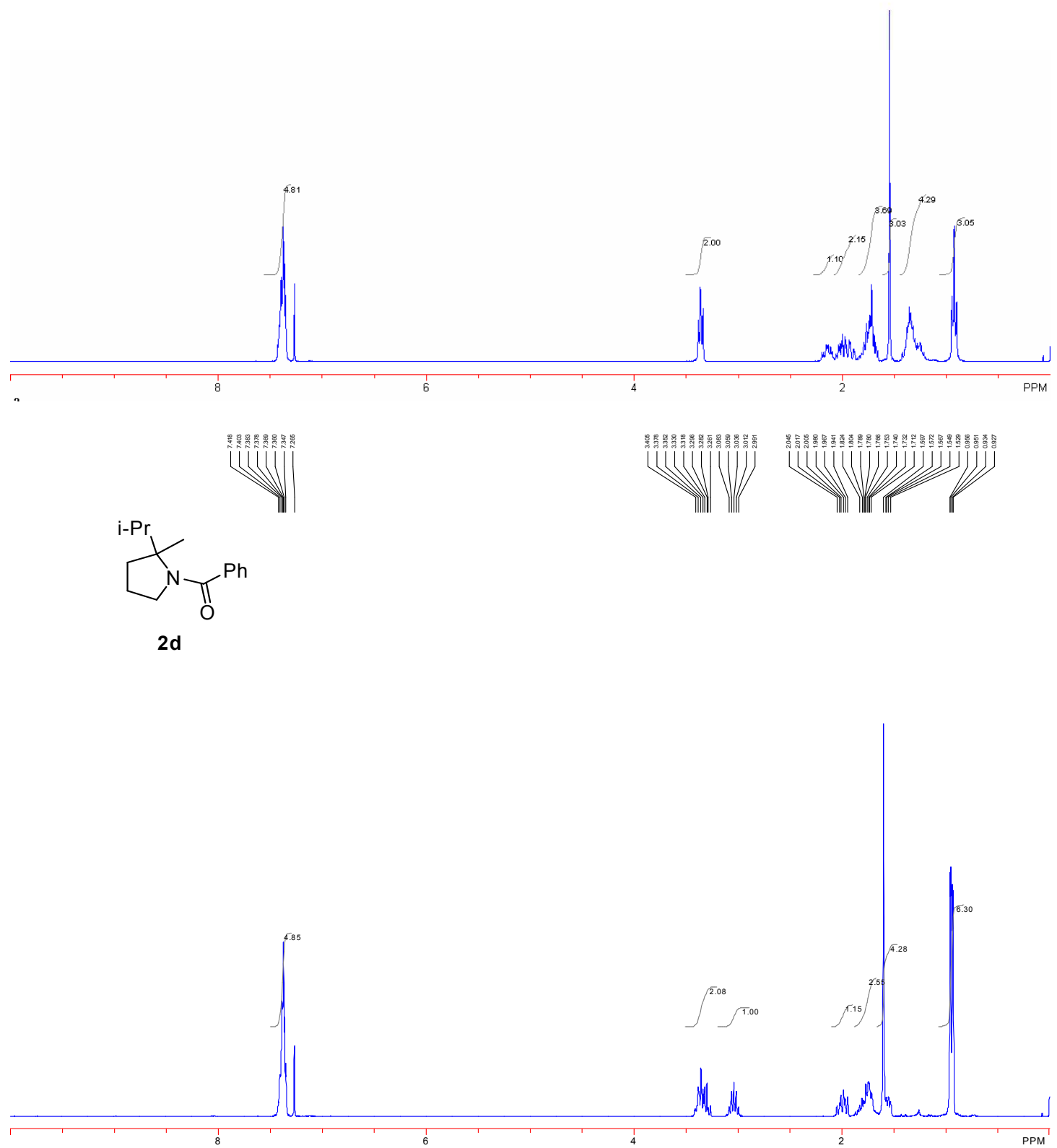

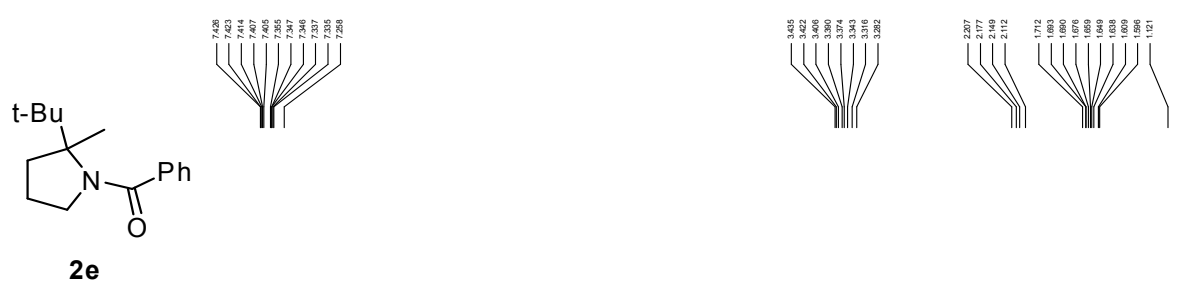

$2 e$
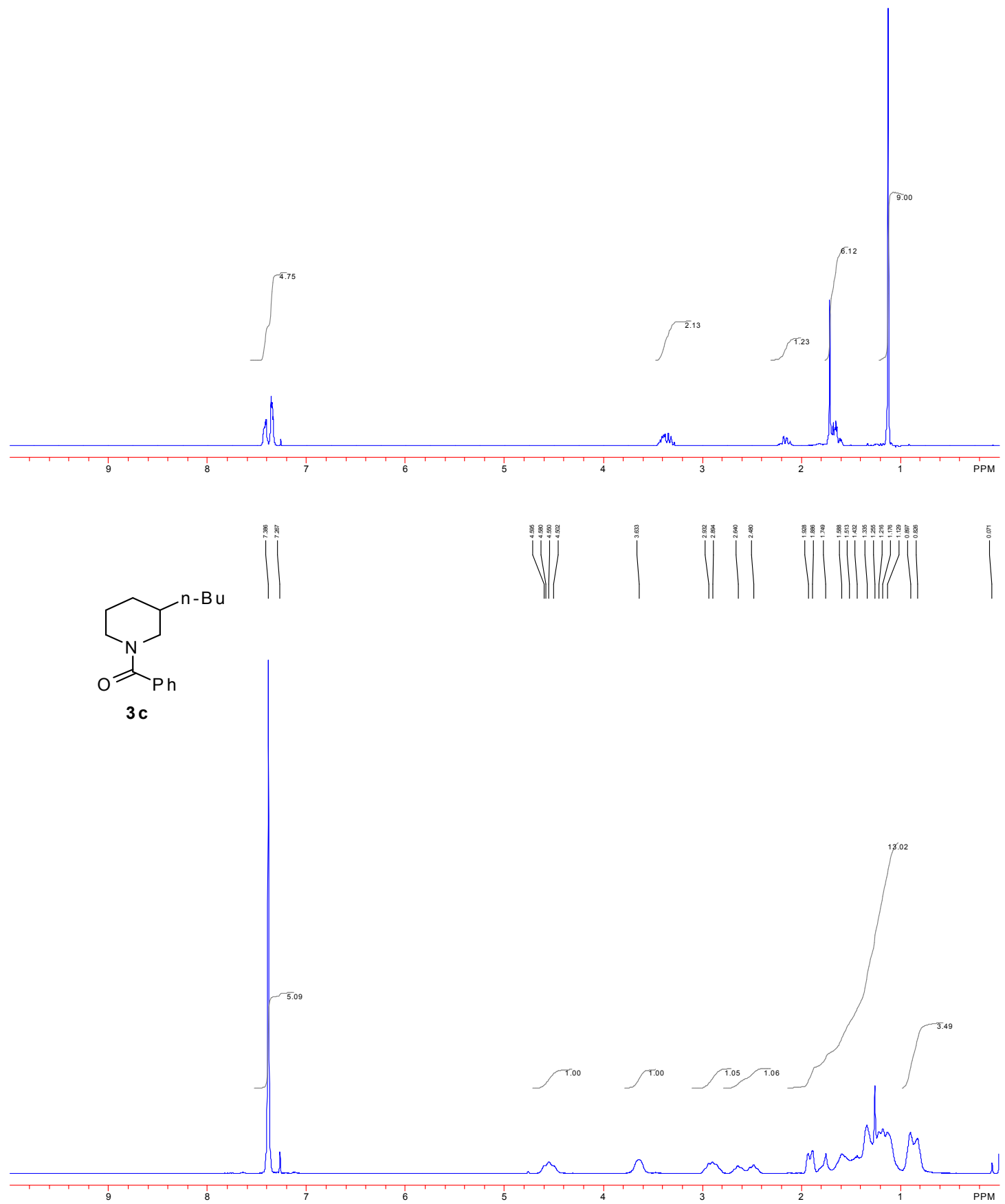


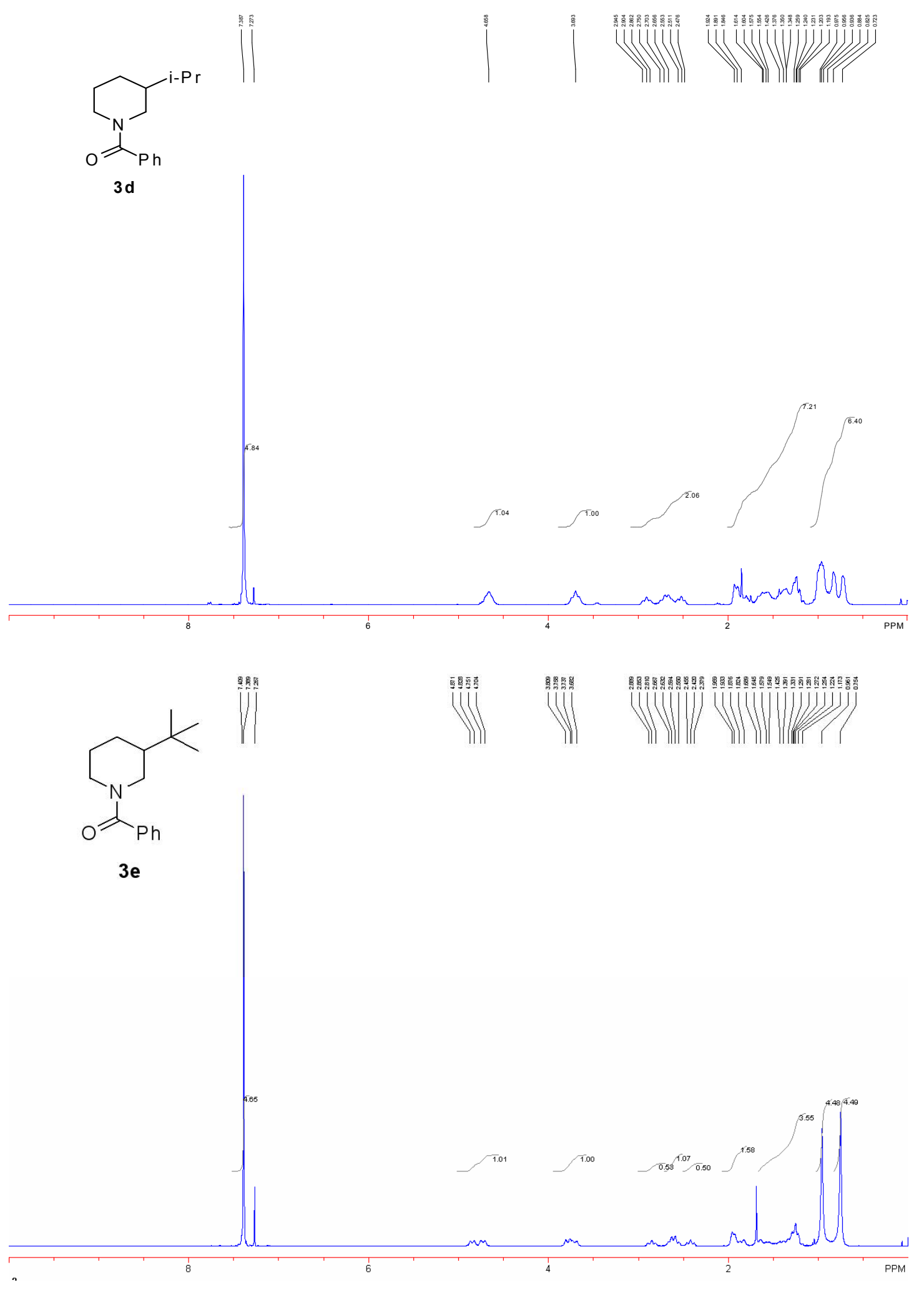



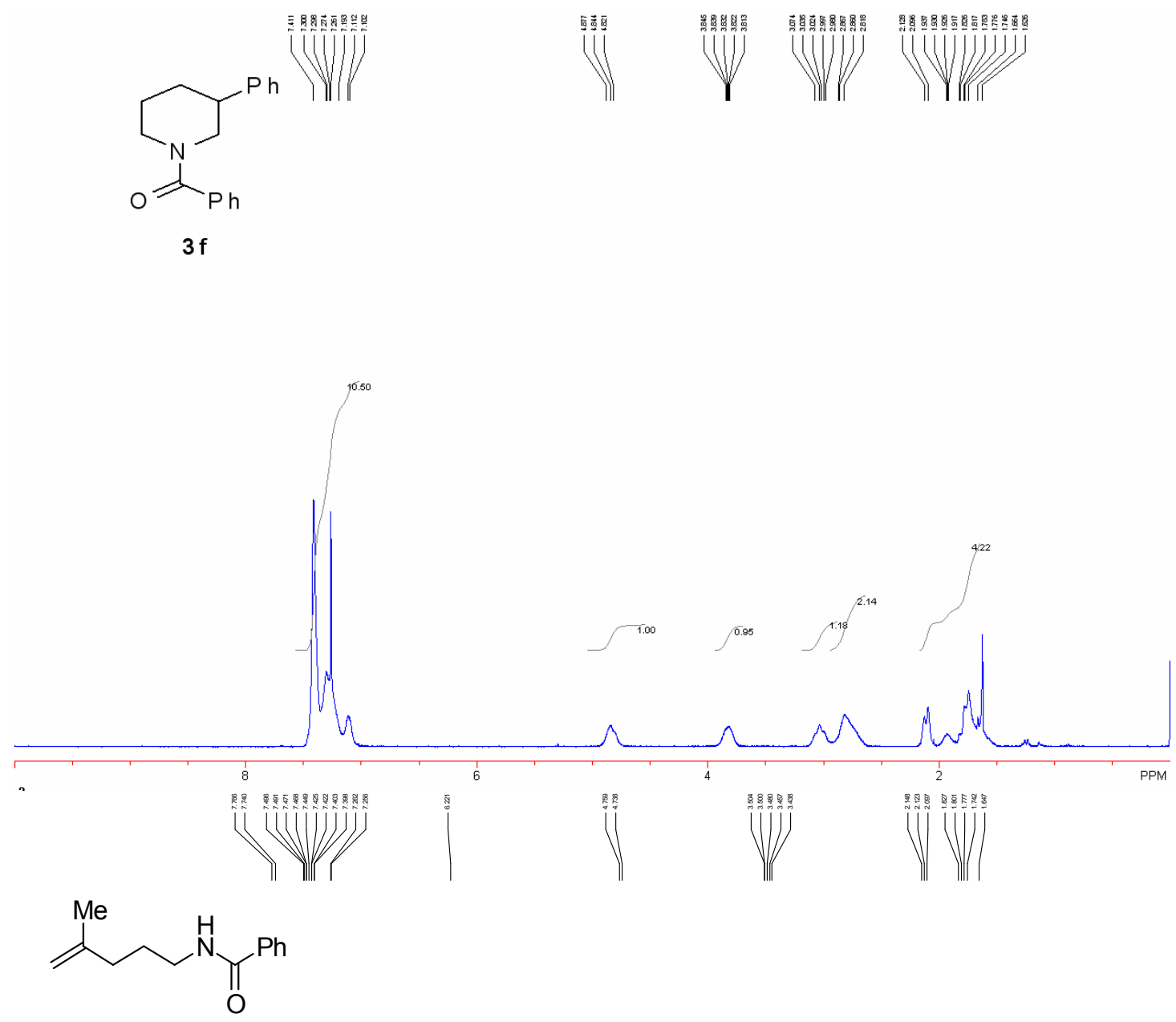

4b

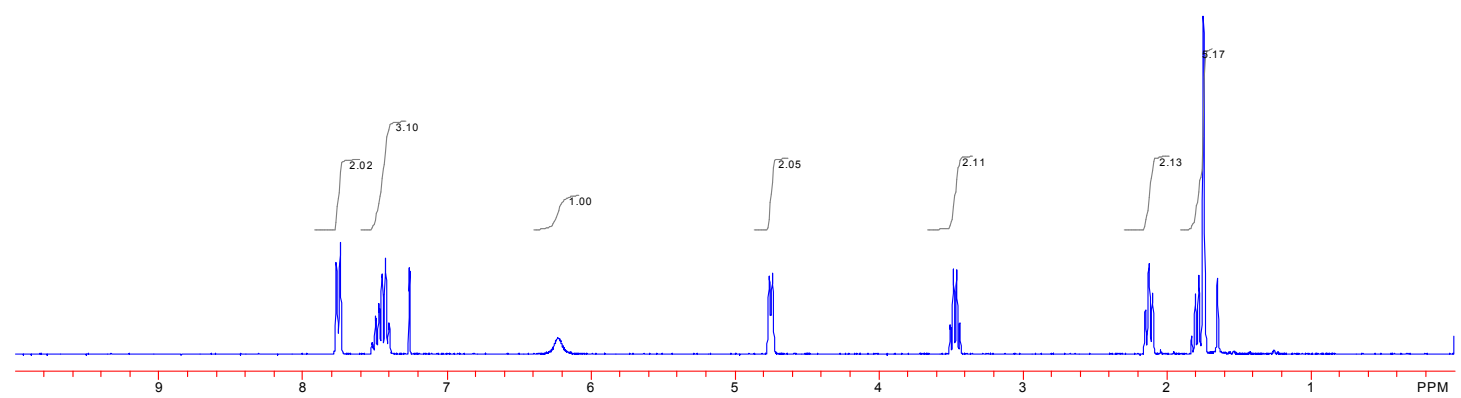



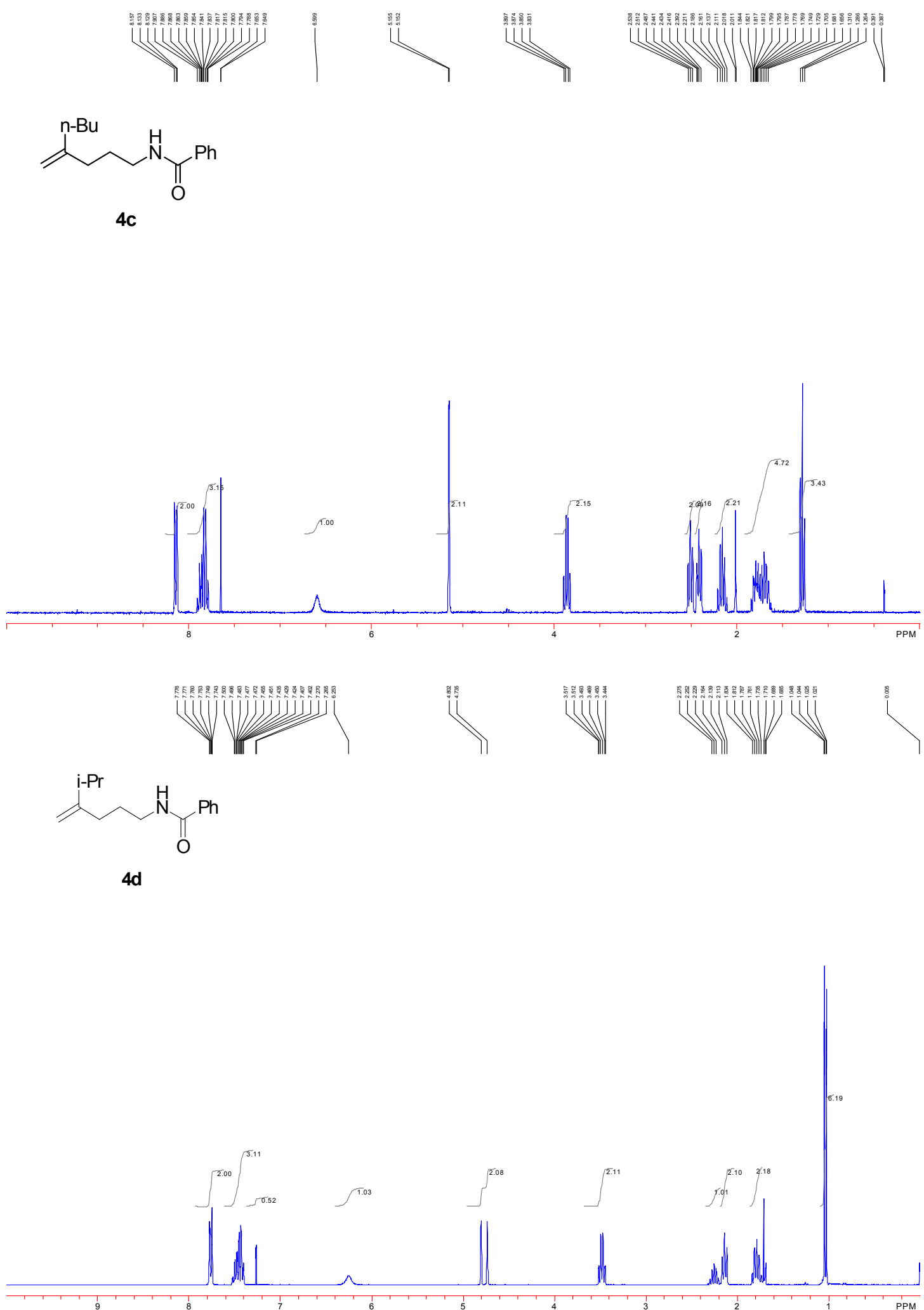

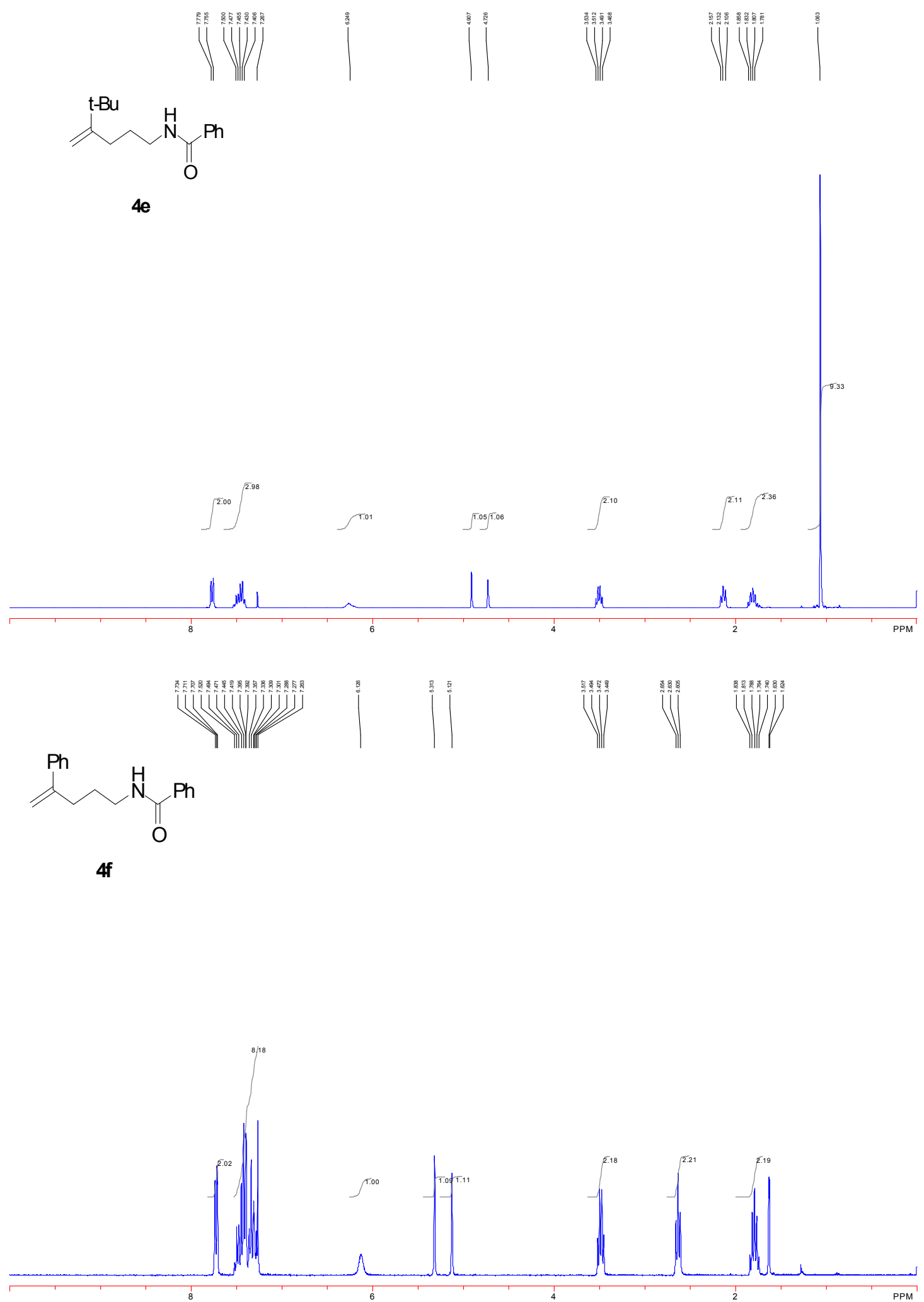

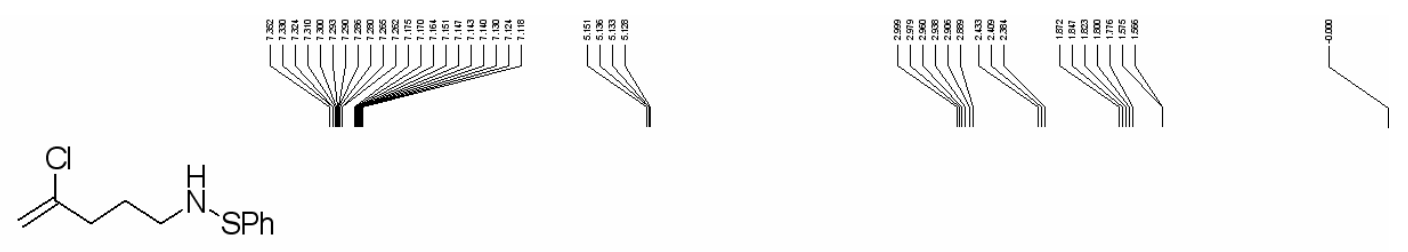

\section{$5 a$}

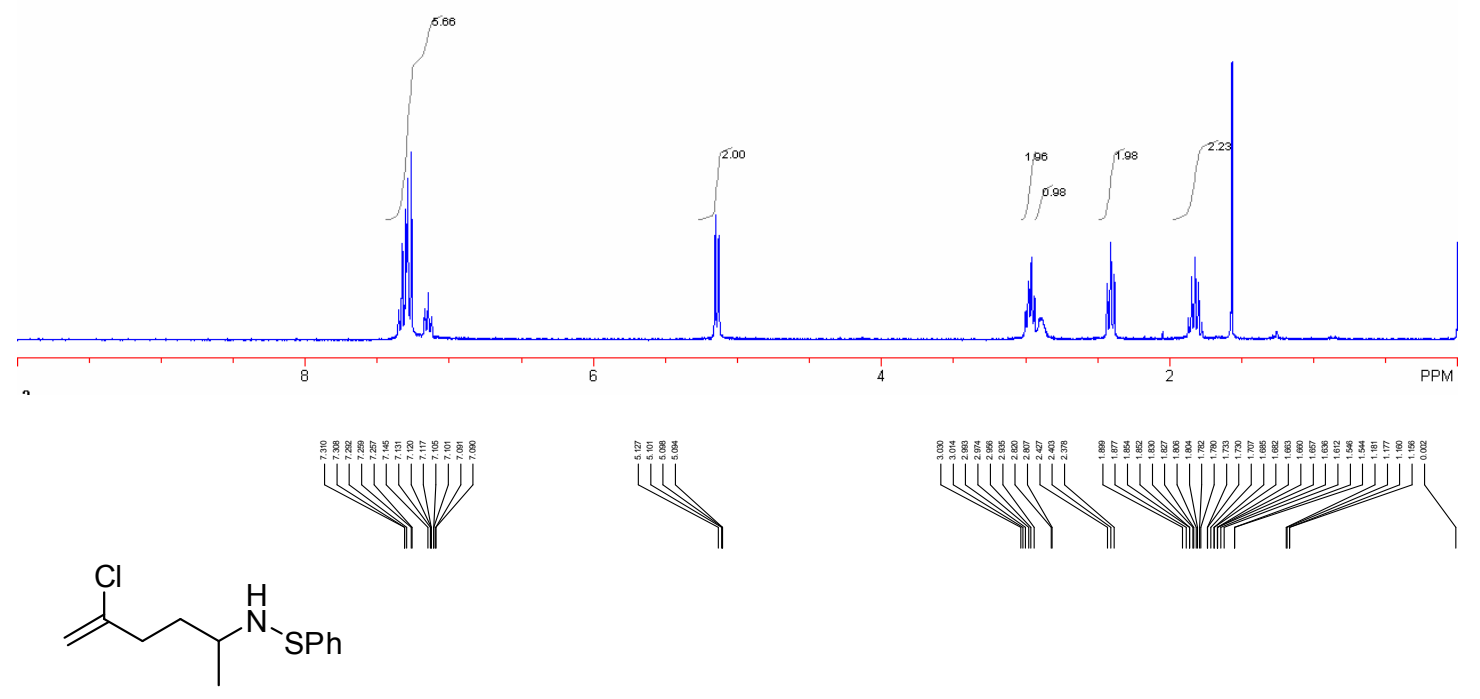

$5 b$

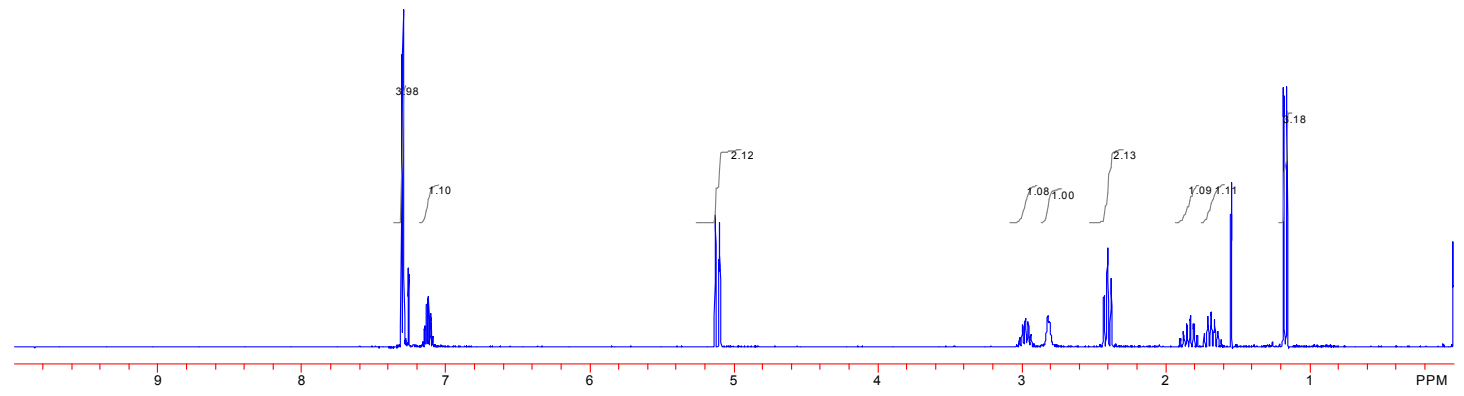



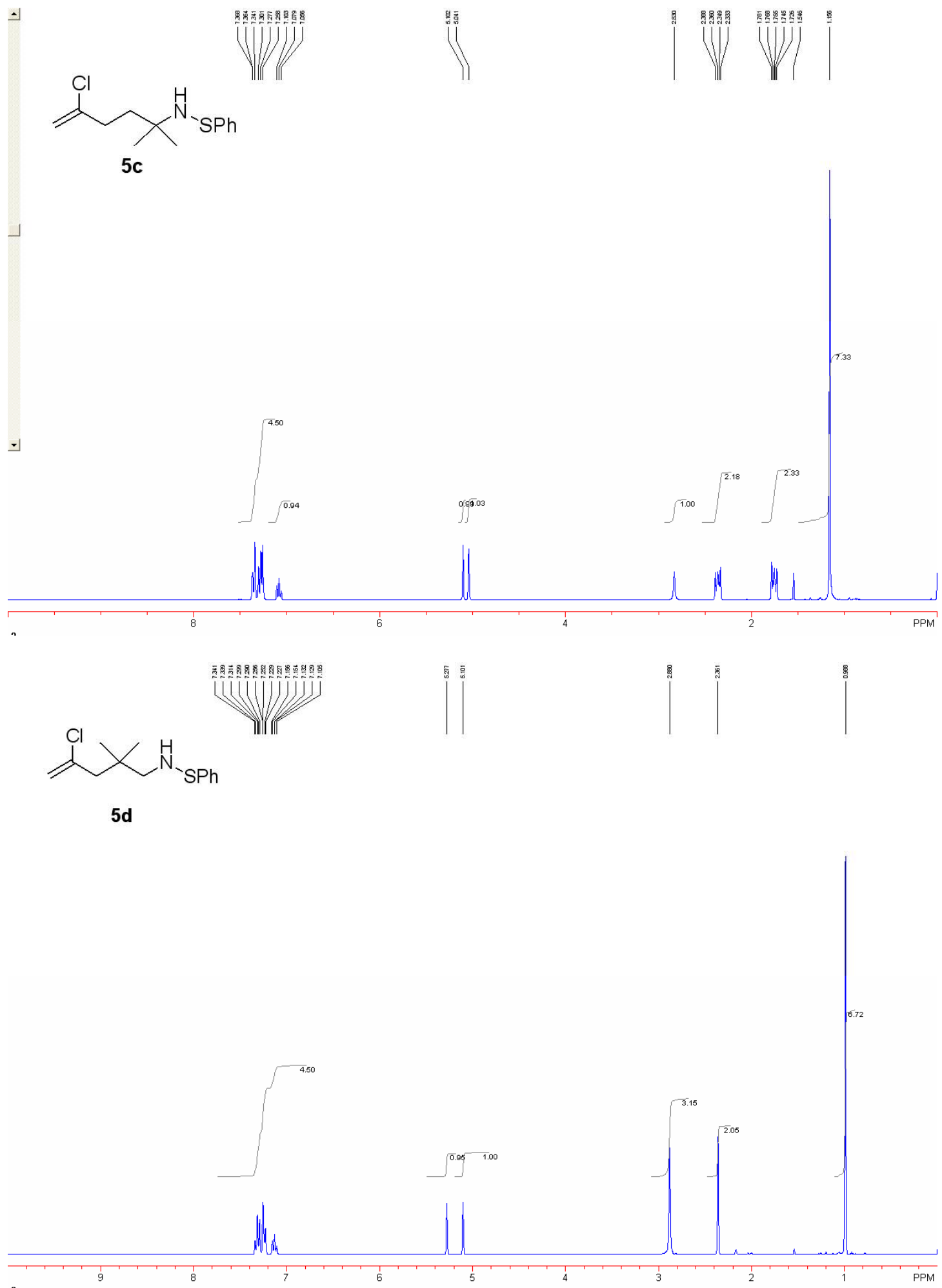

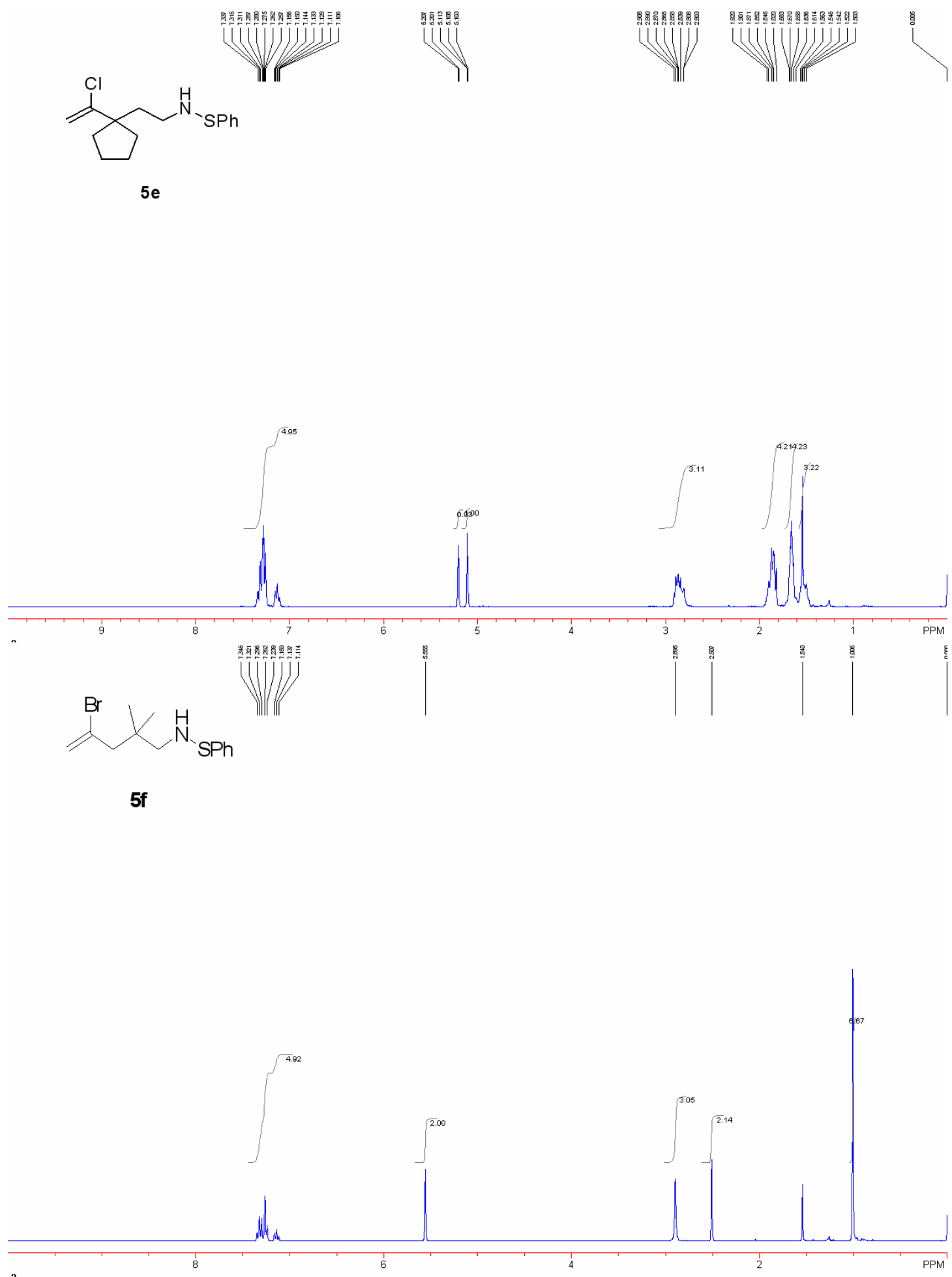

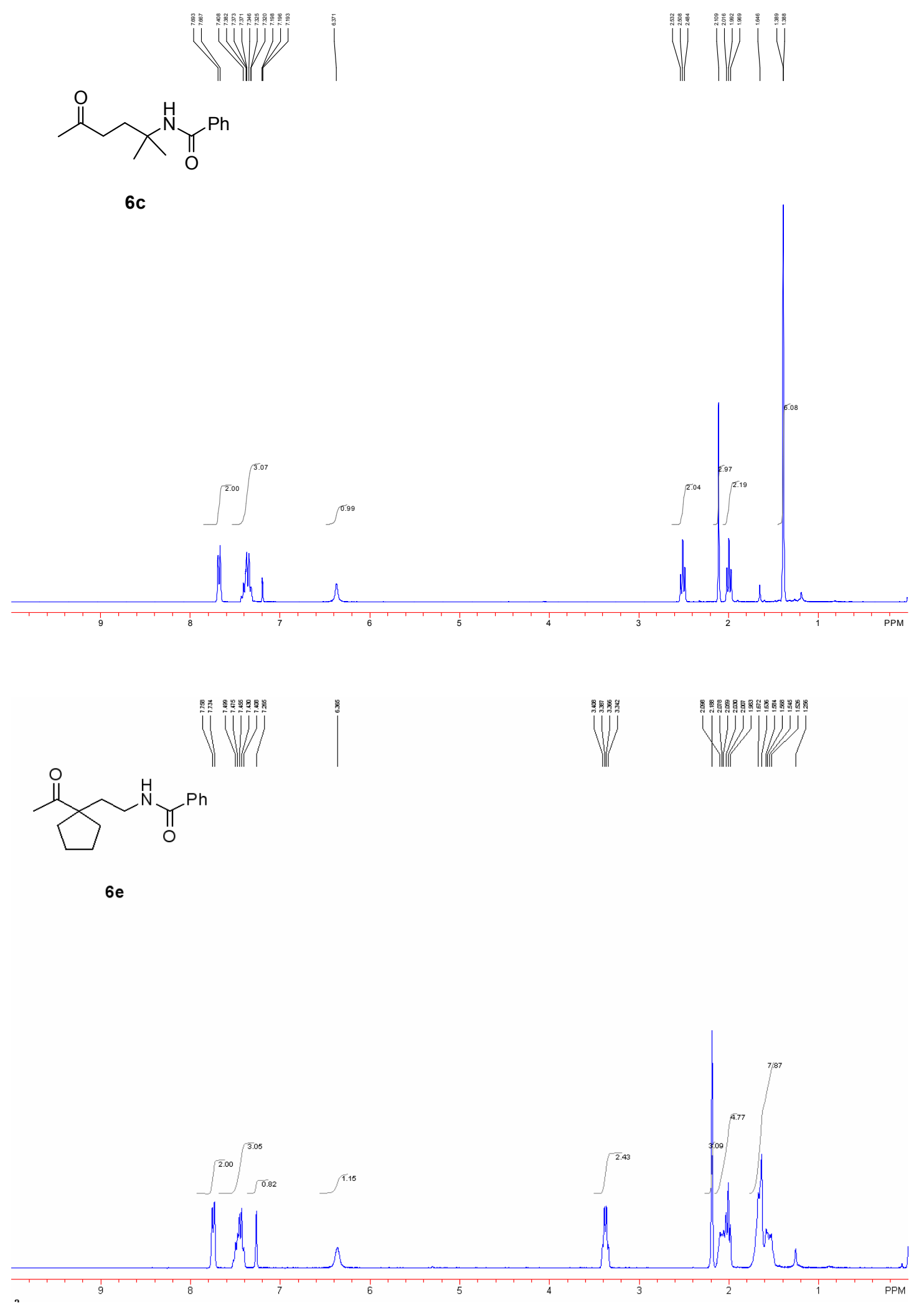

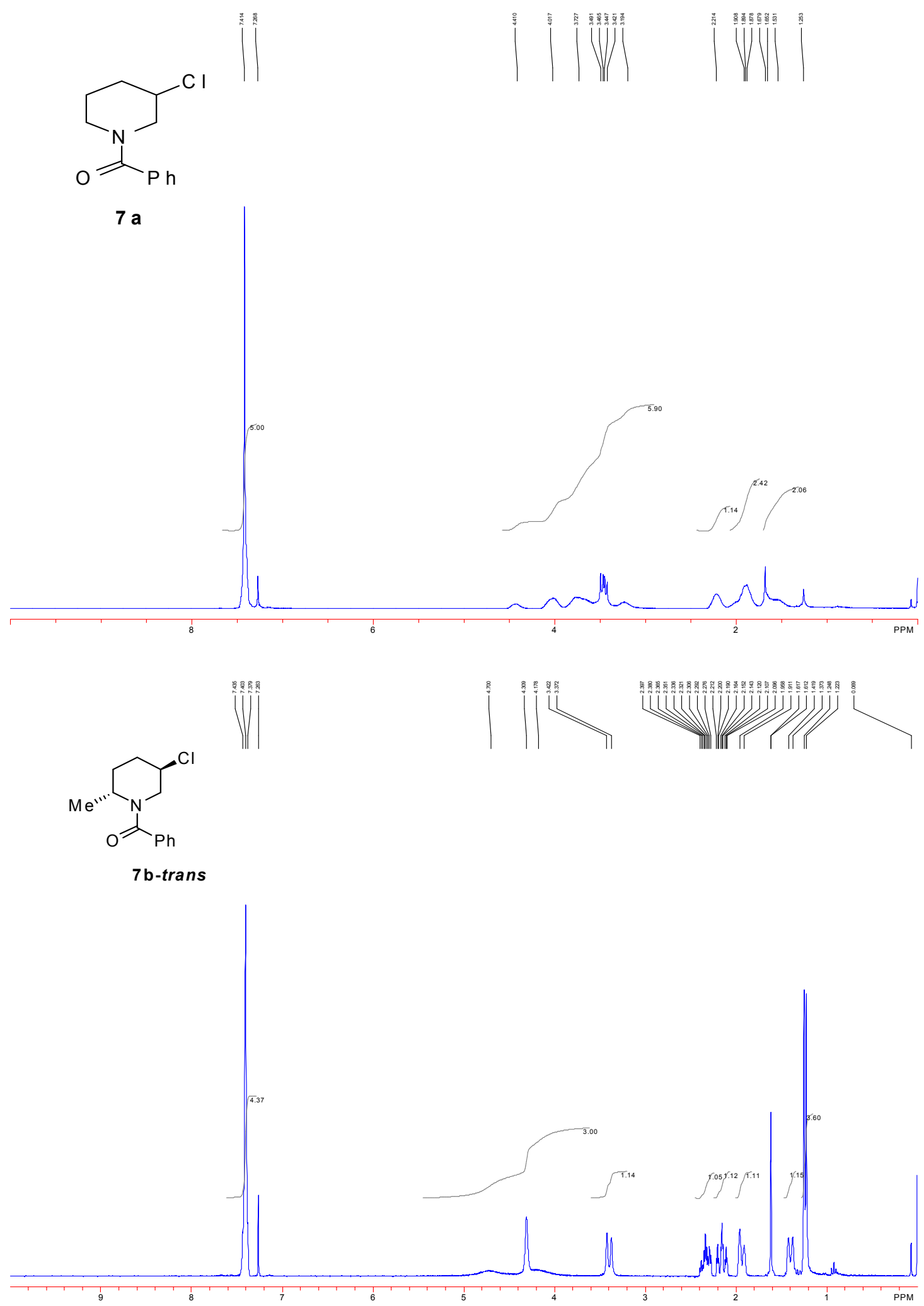

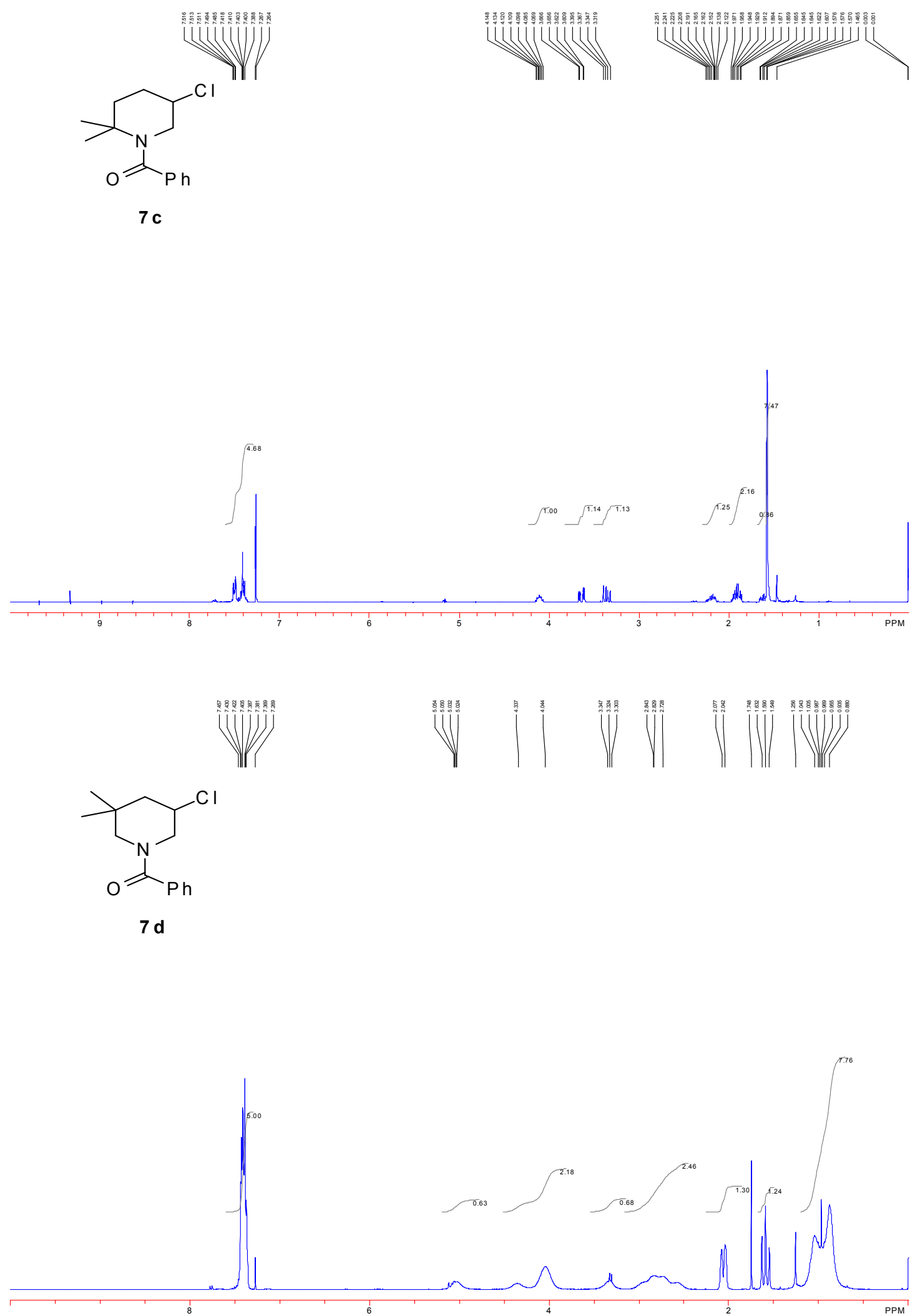


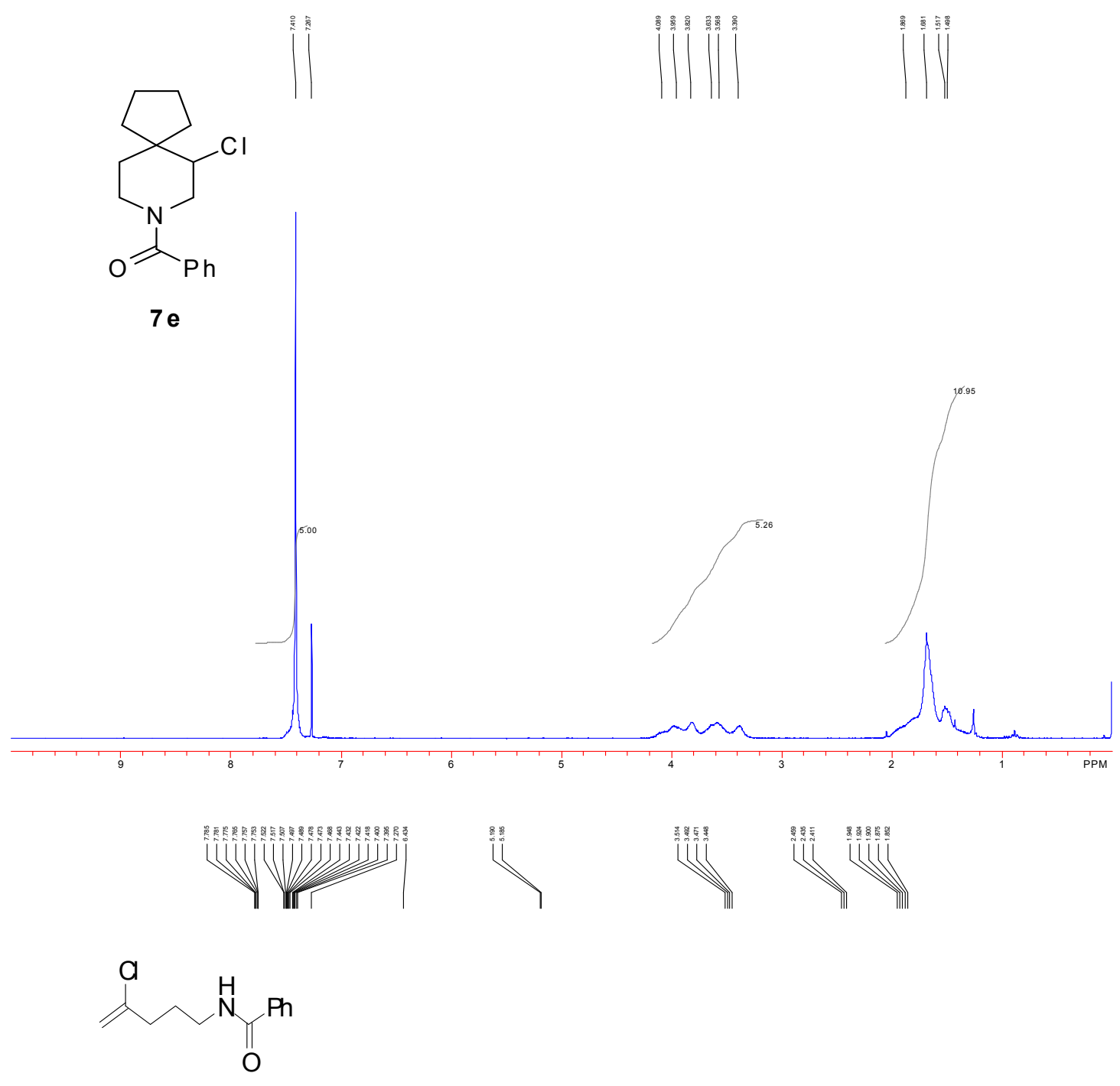

$8 a$

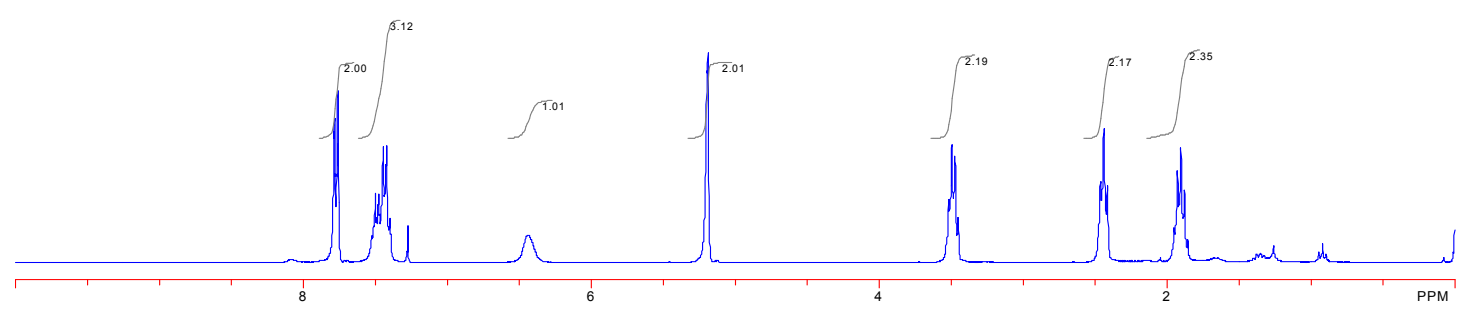



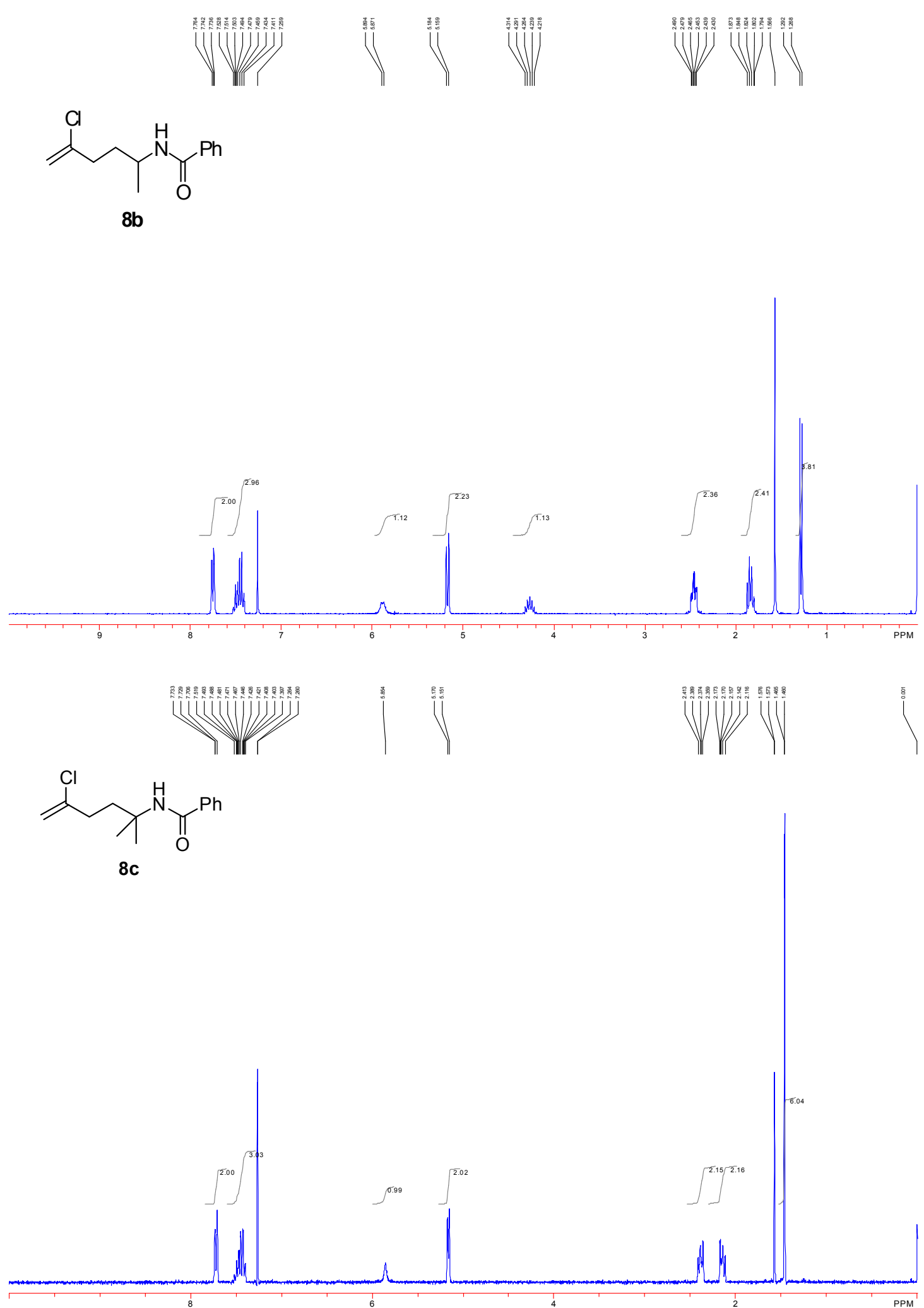


\section{WW}

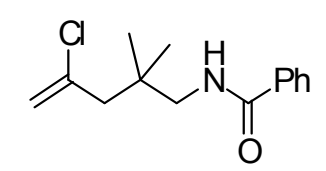

8d
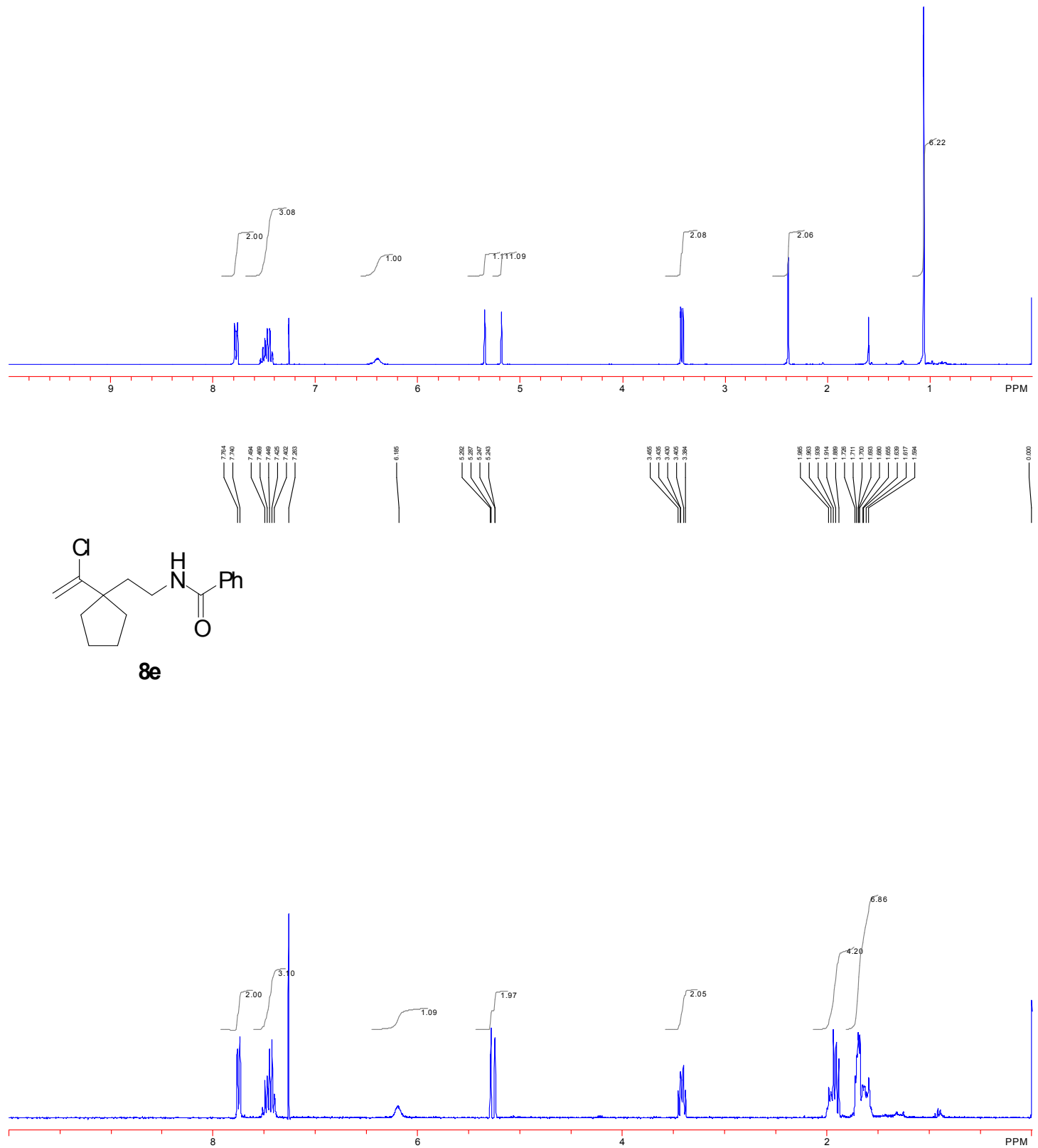

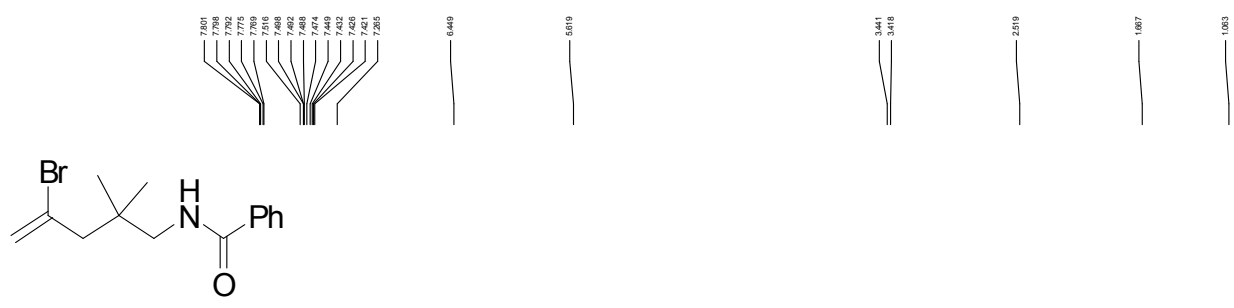

$8 f$

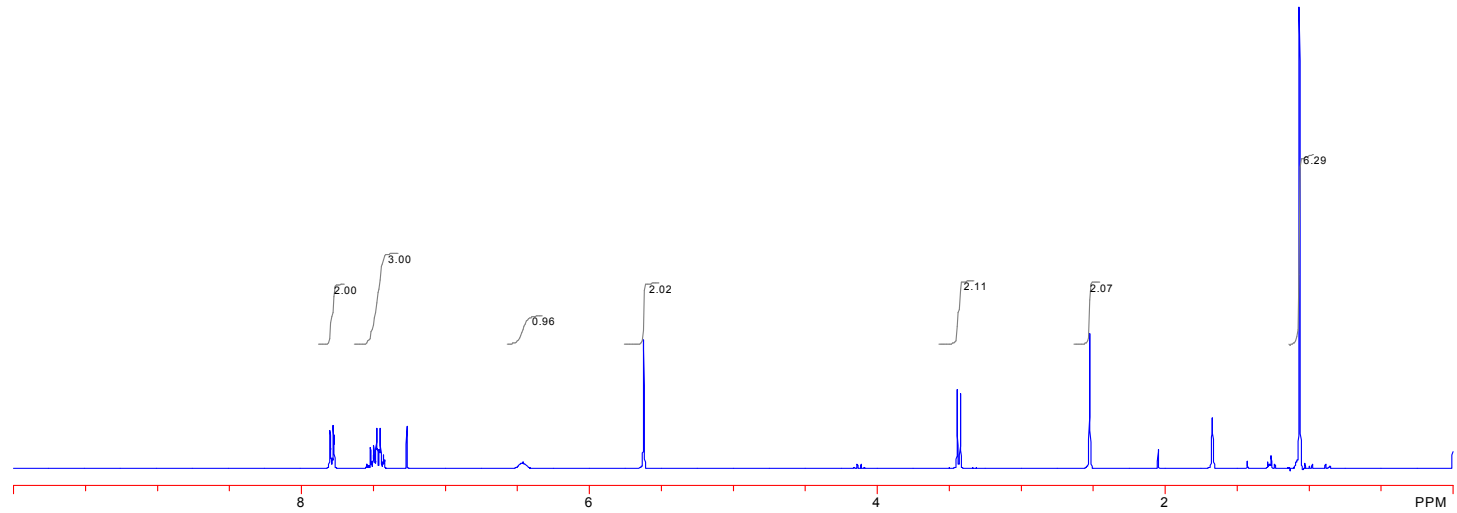

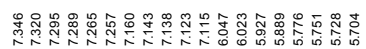

HU)

WII
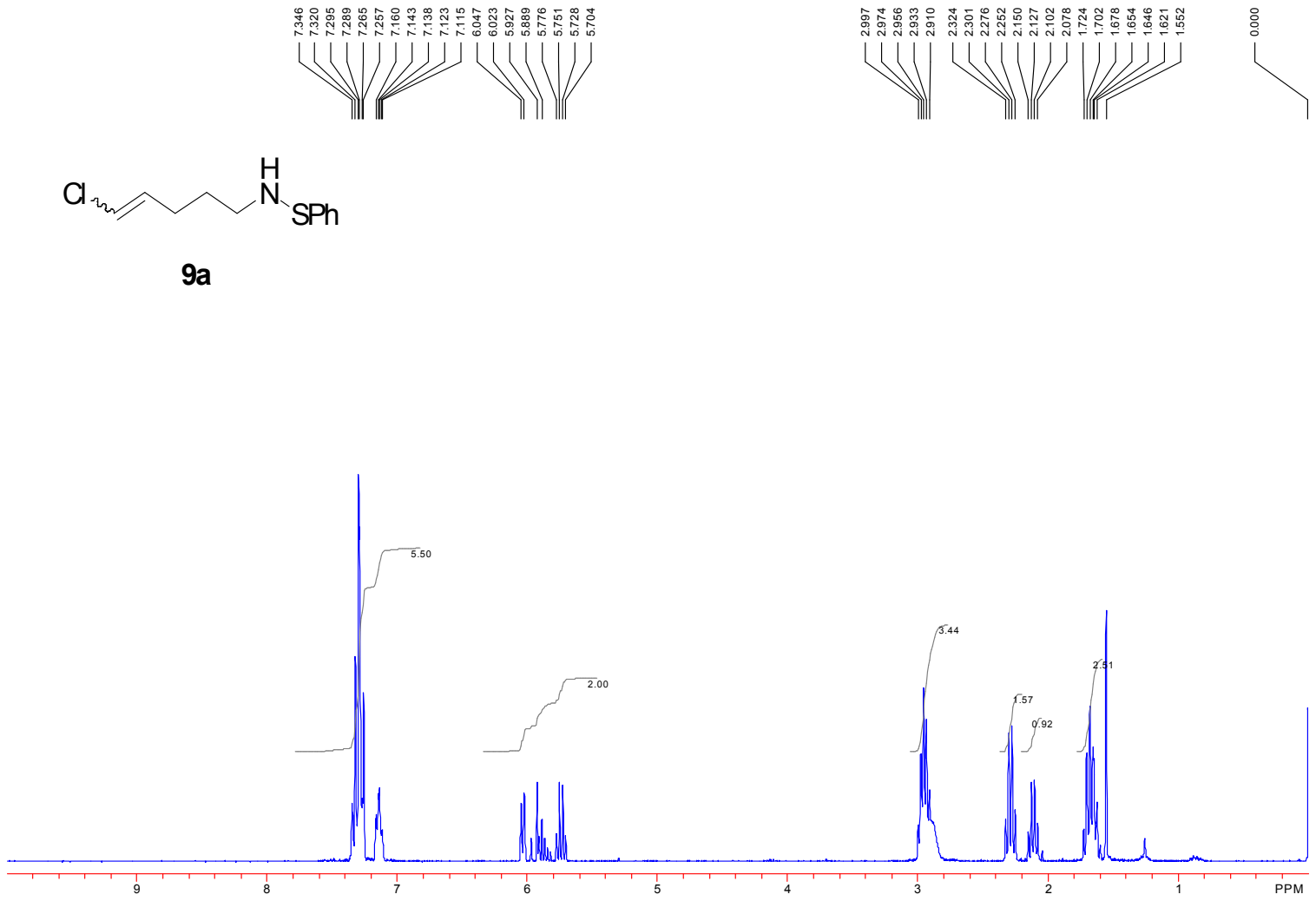

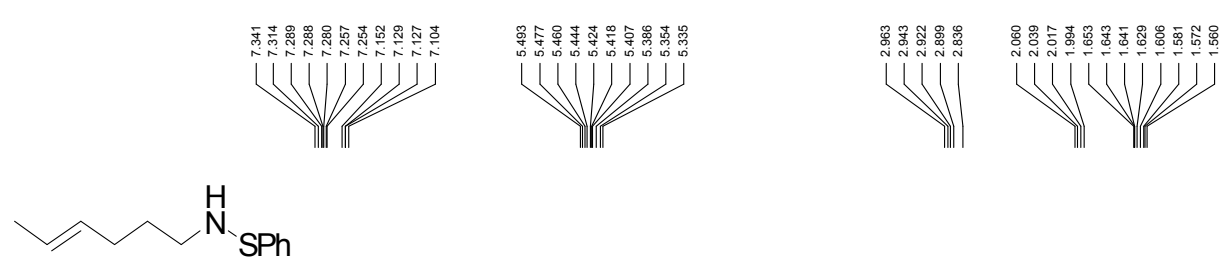

$9 b$
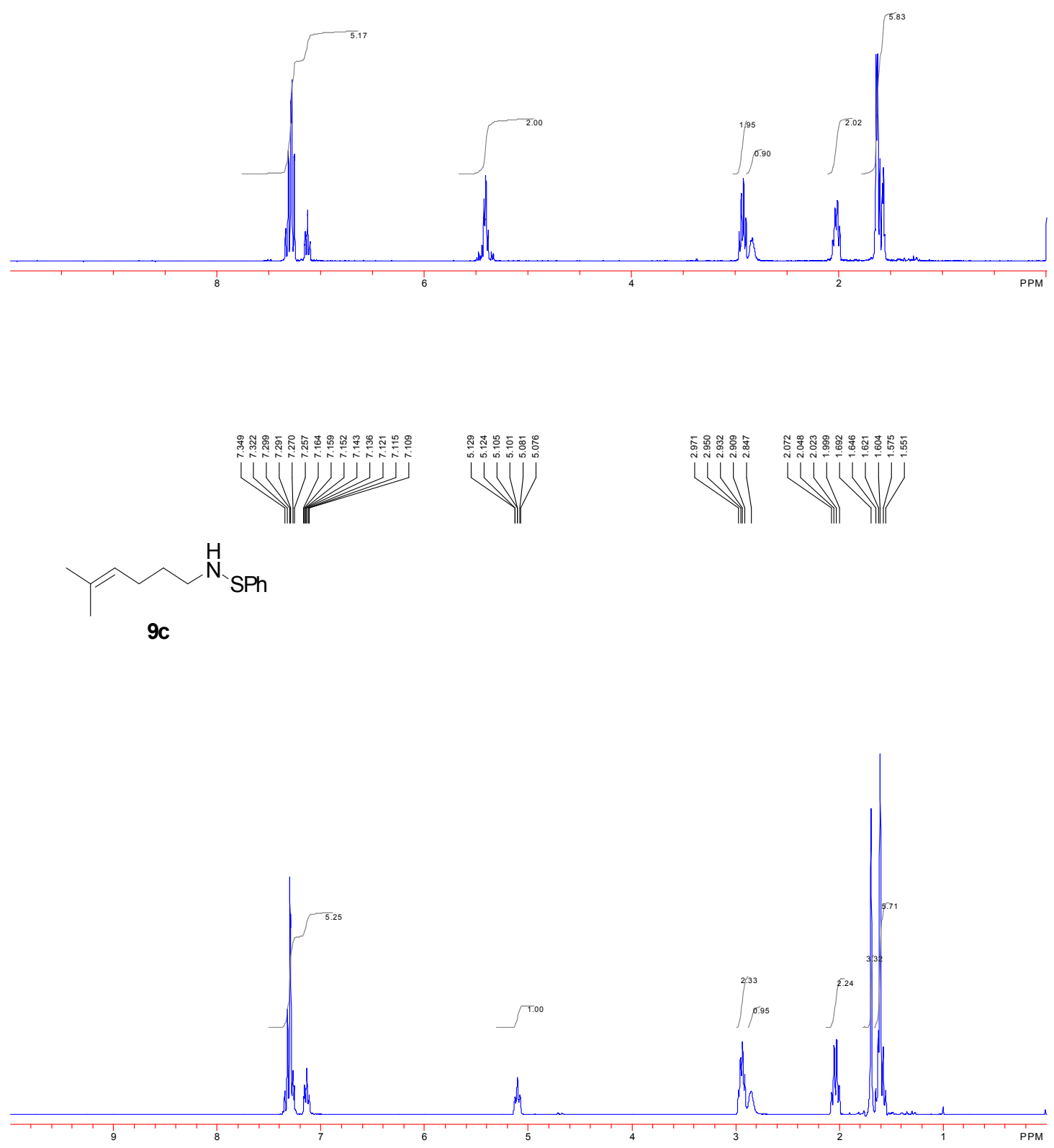

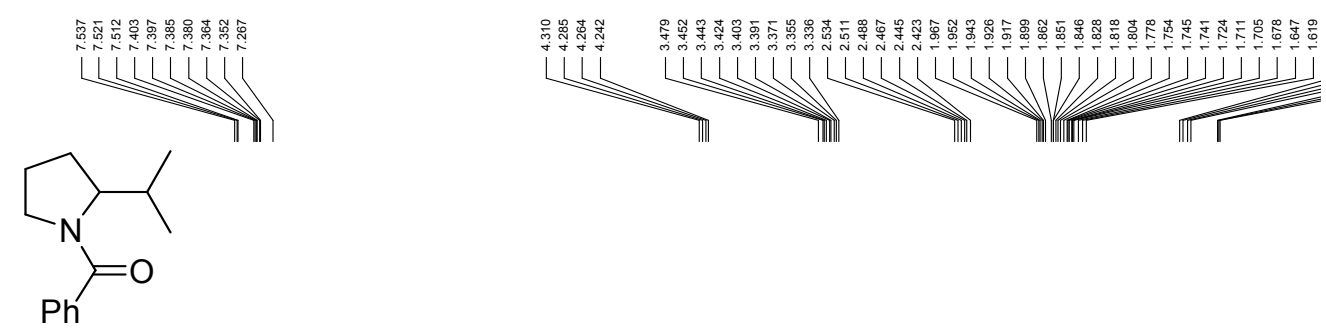

10c

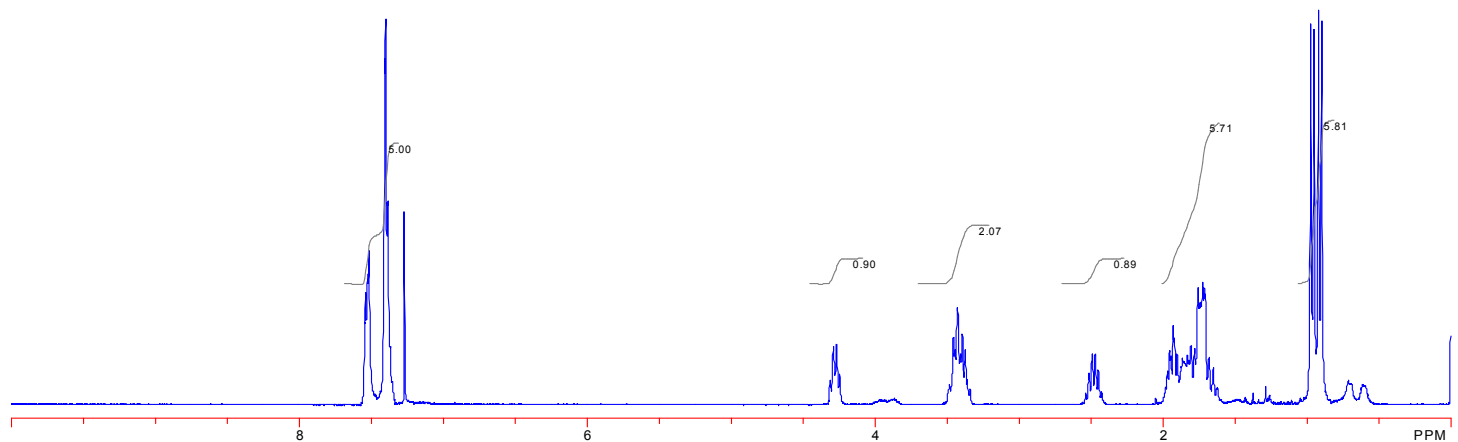

